Western University

Scholarship@Western

Digitized Theses

Digitized Special Collections

1993

\title{
Dynamics Of The Envelopes Of Be Stars In The Equatorial Plane
}

Haiqi Chen

Follow this and additional works at: https://ir.lib.uwo.ca/digitizedtheses

\section{Recommended Citation}

Chen, Haiqi, "Dynamics Of The Envelopes Of Be Stars In The Equatorial Plane" (1993). Digitized Theses. 2198.

https://ir.lib.uwo.ca/digitizedtheses/2198

This Dissertation is brought to you for free and open access by the Digitized Special Collections at Scholarship@Western. It has been accepted for inclusion in Digitized Theses by an authorized administrator of Scholarship@Western. For more information, please contact tadam@uwo.ca,

wlswadmin@uwo.ca. 
The author of this thesis has granted The University of Western Ontario a non-exclusive license to reproduce and distribute copies of this thesis to users of Western Libraries. Copyright remains with the author.

Electronic theses and dissertations available in The University of Western Ontario's institutional repository (Scholarship@Western) are solely for the purpose of private study and research. They may not be copied or reproduced, except as permitted by copyright laws, without written authority of the copyright owner. Any commercial use or publication is strictly prohibited.

The original copyright license attesting to these terms and signed by the author of this thesis may be found in the original print version of the thesis, held by Western Libraries.

The thesis approval page signed by the examining committee may also be found in the original print version of the thesis held in Western Libraries.

Please contact Western Libraries for further information:

E-mail: libadmin@uwo.ca

Telephone: (519) 661-2111 Ext. 84796

Web site: http://www.lib.uwo.ca/ 
DYNAMICS OF THE ENVELOPES OF Be STARS IN THE EQUATORIAL PLANE

by

Kaiqi Chen

Department of Astronomy

Submitted in partial fulfilment of the requirements for the degree of Doctor of Philosophy

Faculty of Graduate Studies

The University of Western Ontario

London, Ontario

September 1992

- Halq1 Chen 1992 
National Library

of Canada

Acquisitions and

Bibliographic Services Branch

395 Wellington Street

Ottawa. Ontano

K1A ON4
Bibliothèque nationale

du Canada

Direction des acquisitions et

des services bibliographiques

395. rue Wellinglon

Ottawa (Ontarno)

KIA ON4 vour the vorre resurence

Our fite Notre ntherence
The author has granted an irrevocable non-exclusive licence allowing the National Library of Canada to reproduce, loan, distribute or sell copies of his/her thesis by any means and in any form or format, making this thesis available to interested persons.
L'auteur a accordé une licence irrévocable et non exclusive permettant à la Bibliothèque nationale du Canada de reproduire, prêter, distribuer ou vendre des copies de sa these de quelque manière et sous quelque forme que ce soit pour mettre des exemplaires de cette thèse à la disposition des personnes intéressées.

L'auteur conserve la propriété du droit d'auteur qui protège sa thèse. Ni la thèse ni des extraits substantiels de celle-ci ne doivent être imprimés ou autrement reproduits sans son autorisation.
The author retains ownership of the copyright in his/her thesis. Neither the thesis nor substantial extracts from it may be printed or otherwise reproduced without his/her permission. 


\section{ABSTRACT}

In this thesis, I shall investigate the dynamics of the envelopes of Be stars in the equatoria! plane. Currently there is no general agreement as to what are the most important forces controlling the dynamics in and near the equatorial plane. I expect that my research will yield a significant improvement in the knowledge of the forces which are most important in controlling the dynamics of the circumstellar matter, and thus lead to a major advance in the understanding of Be stars. Recently, observations of the continuous energy distribution of several Be stars from infrared to radio wavelengths have been analyzed using simple models in order to determine both the density distribution and the radial component of velocity in the emitting matter. Earlier studies of spectral line profiles from Be stars also gave information about the velocity field. Both types of investigation have provided essential data for my study of the dynamics. In my approach I invert the equation of motion and solve for the unknown force or forces, $F_{x}(x)$, in addition to gravitation, rotation, and gas pressure gradient, required to produce a radial component of velocity having a functional form consistent with the data mentioned above. All of my investigations indicate that $F_{x}(r)$ has a similar characteristic shape. Beginning at the surface of the $s t a r, F_{k}(r)$ initially decreases with increasing $r$, but less rapldiy than does gravity. reaches a minimum at $10-100$ stellar radif, and then increases again. It may continue to increase or reach a maximum and then decrease again, depending upon the detailed form of the velocity distribution used. This minimum in $F_{x}(r)$ and the subsequent increase beyond the minimum suggest either a change in the strength of $F_{x}$, due perhaps to varying physical properties, and/or some other physical effect becoming important. 
For a small sample of 3 Be stars and 3 Be-shell stars, I have noted that the minimum of $F_{x}(r)$ for the Be stars is closer to the stellar surface than is that for the 3 Be-shell stars. This distinction may reflect the small sample size, or it may be a real physical effect, indicating that the denser part of the envelopes of Be-shell stars are more extensive than those of Be stars.

I next investigated how a weak radial magnetic field influences the dynamics. My investigation has shown that in a slowly expanding envelope, a weak magnetic field can influence the rotational -elocity distribution quite effectively so that the centrifugal force is enhanced. For some cases the centrifugal force is so large that it can balance gravity.

I then considered another promising driving mechanism for Be star envelopes, namely, the radiation force due to the optically thin lines. I introduced two parameters, $\epsilon$ and $\eta$, to describe this force, and constructed a weak line, radiation driven wind model. My calculations have shown that the model can also produce a slowly expanding wind with a terminal velocity of order of $60 \mathrm{~km} / \mathrm{s}$. When I used the velocity distribution and PM model to calculate the Ho profile, I obtained a reasonably good profile. 
at $\mathrm{H} \beta, \mathrm{H \gamma}$, and so on up the Balmer serles. For some stars emission is only visible at Ha.

The emission may appear as a single peak or as double peaks with a central reversal. When double emission peaks are present, the redward peak and the blueward peak are referred to as $R$ and $V$ components, respectively. The ratio of $V$ to $R, V / R$, is an important parameter to describe the asymmetry of emission profiles. When the $V$ component is stronger than $R$, we say $V / R>1$, otherwise, $V / R<1$.

\subsubsection{Polarization}

Another characteristic property of Be stars is their intrinsic linear polarization. The degree of polarization in the continuum is of order 1 percent, and generally does not exceed 2 percent. The fact that it is only detected for Be stars but not for normal B type stars suggests that the polarization originates in the envelopes of Be stars. Although the polarization is not high, it provides strong evidence for the asphericity of the envelopes of Be stars.

\subsubsection{Rotational Velocities of Be Stars}

Many observations of rotational velocities of Be ztars have been carried out. The revised Catalogue of Stellar Rotational Velocities by Uesugi and Fukuda (1982) contains all published values of stellar projected rotational velocities, $v$ sin $i$. There is a systematic difference between rotational velocities of Be stars and those of normal B type stars (Harmanec 1982). Compared with normal B type stars, Be stars as a class are much faster rotators. However, the rotational velocities of Be stars are still below the critical velocity, $v_{\text {cri }}$, which is defined 


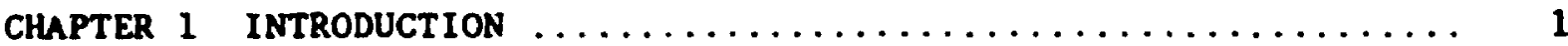

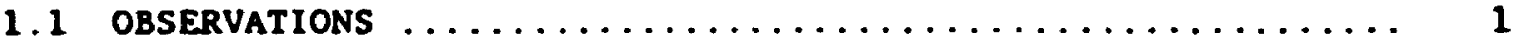

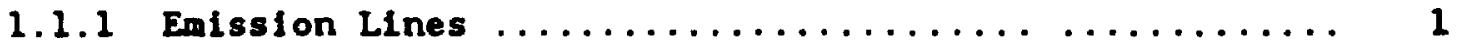

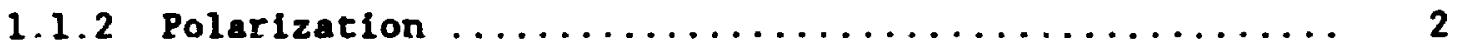

1.1.3 Rotational Velocities of Be Stars .............. 2

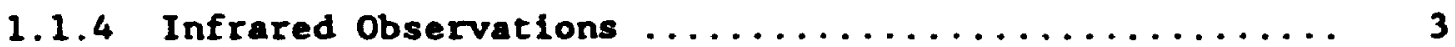

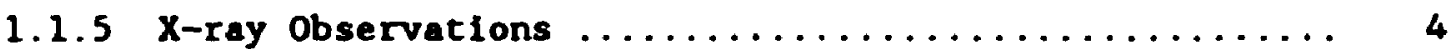

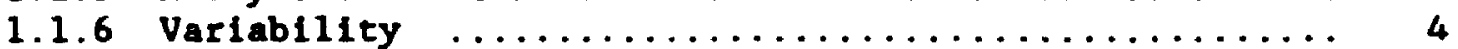

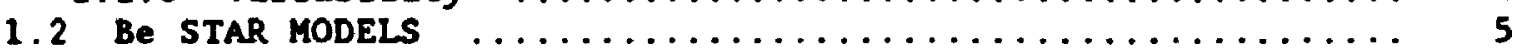

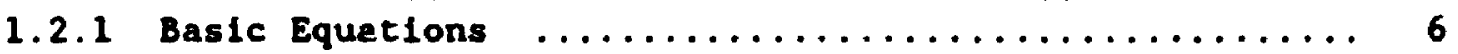

1.2 .2 Struve's Rotational Model $\ldots \ldots \ldots \ldots \ldots \ldots \ldots \ldots \ldots \ldots \ldots$

1.2.3 The PH Model ........................... 7

1.2.4 A Radiation Driven Wind Model due to Optically Thick Lines ..................... 8

1.2.5 Thomas \& Doazan Model ..................... 11

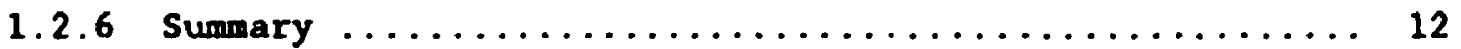

CHAPTER 2 WHICH FORCE/FORCES CONTROL(S) THE DYNAMICS OF THE ENVELOPES IN THE EQUATORIAL PLANE ............ 13

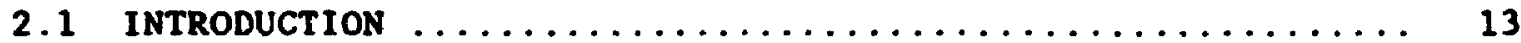

2.2 FUNCTIONAL FORMS OF THE VELOCITY LAWS $\ldots \ldots \ldots \ldots \ldots \ldots \ldots \ldots$

2.3 DYNAMICS IN THE EQUATORIAL PLANE $\ldots \ldots \ldots \ldots \ldots \ldots \ldots \ldots \ldots$

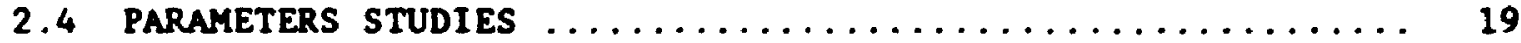

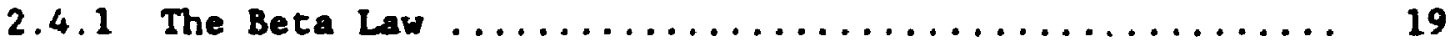

2.4.1.1 Angular Momentun Conservation ............. 21

2.4.1.2 Keplerian Rotation .................... 24

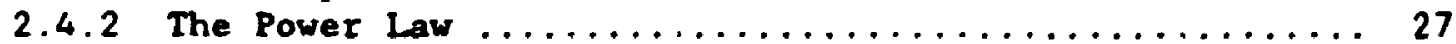

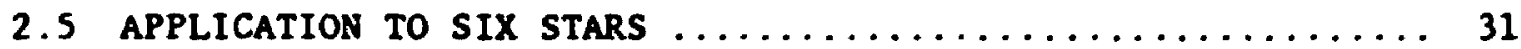

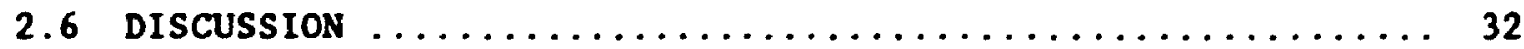

CHAPTER 3 A POTENTIAL SIMILARITY AND DIFFERENCE BETWEEN Be STARS

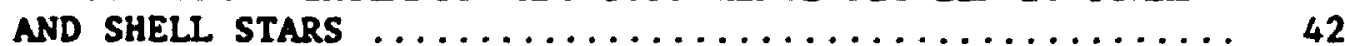

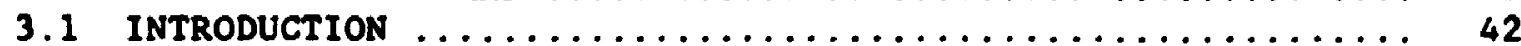

$3.2 F_{z}$ FOR THREE Be STARS AND THREE SHELL STARS $\ldots \ldots \ldots \ldots \ldots \ldots .43$

3.3 DISCUSSION .............................. 46

CHAPTER 4 THE INFLUENCE OF A WEAK MAGNETIC FIELD ON THE AZIMUTHAL MOTION OF A SLOHLY EXPANDING WIND ......... 50

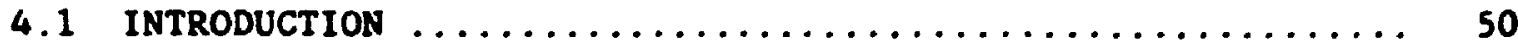

4.2 DYNAMICS IN THE EQUATORIAL PLANE WHEN A MAGNETIC FIELD

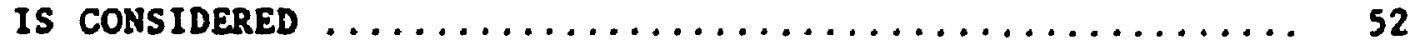

4.3 DISTRIBUTION OF THE AZIMUTHAL VELOCITY AND MAGNETIC FIELD.. 55

4.3.1 The Beta Law Velocity.................... 56

4.3.2 The Power Lav Velocity Distribution ............ 62

4.4 WHAT $F_{\times}$DO WE NEED? $\ldots \ldots \ldots \ldots \ldots \ldots \ldots \ldots \ldots \ldots \ldots \ldots \ldots \ldots$ 
CHAPTER 5 WINDS DRIVEN BY OPTICALLY THIN LINES

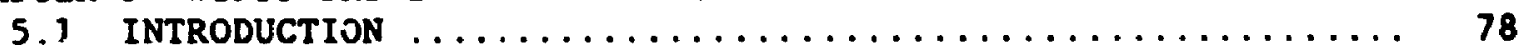

5 THE FORCE DUE TO OPTICALIY THIN LINES $\ldots \ldots \ldots \ldots \ldots \ldots \ldots$

5.3 THE DYNAMICS IN THE EQUATORIAL PLANE $\ldots \ldots \ldots \ldots \ldots \ldots \ldots$.

5.3.1 The Equation of Motion ................... 81

5.3.2 The Determination of $y_{\text {, }}$ and $\operatorname{RIX}(x) \ldots \ldots \ldots \ldots \ldots$

5.3.3 The Determination of $W(x)$ and $W I X(x) \ldots \ldots \ldots \ldots 5$

5.4 ANALYTIC ANALYSIS OF THE DYNAMIC EQUATION $\ldots \ldots \ldots \ldots \ldots \ldots$

5.4 .1 The Restriction for the Values of $c$ and $\eta \ldots \ldots \ldots \ldots 8$

5.4 .2 Terminal Velocity ..................... 89

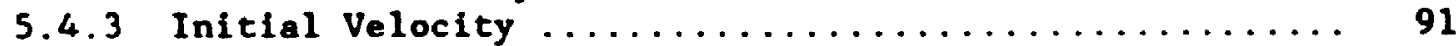

5.5 VELOCITY DISTRIBUTION CALCULATION $\ldots \ldots \ldots \ldots \ldots \ldots \ldots \ldots \ldots$

5.6 APPLICATION TO Be STARS $\ldots \ldots \ldots \ldots \ldots \ldots \ldots \ldots \ldots \ldots \ldots \ldots \ldots$

CHAPTER 6 CONCLUSIONS AND FUTURE WORK $\ldots \ldots \ldots \ldots \ldots \ldots \ldots \ldots \ldots$

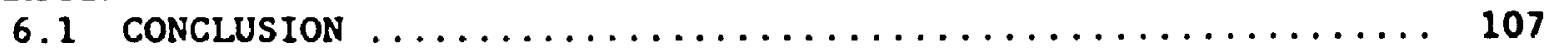

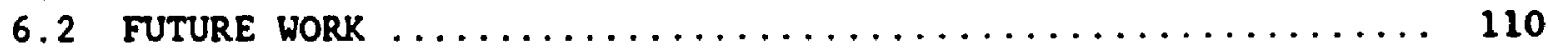

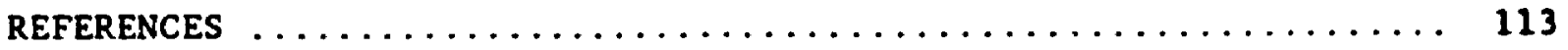

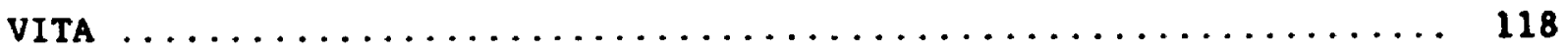




\section{LIST OF TABLES}

Table

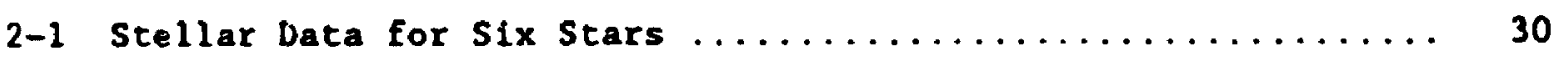

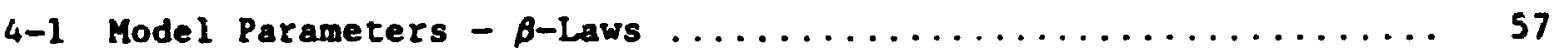

4-2 Model Parameters - Power Laws with Fixed ............ 63

4-3 Model Parameters - Power Laws with Fixed Bo ........... 63

4-4 Model Parameters - Power Laws with Fixed Mass Loss Rate ..... 64

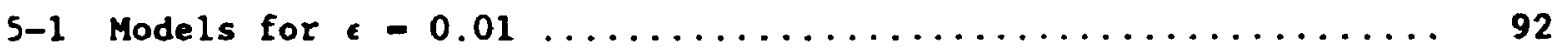

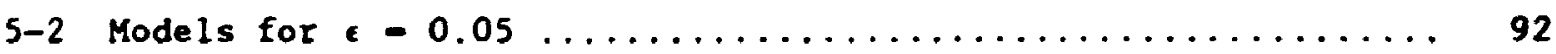

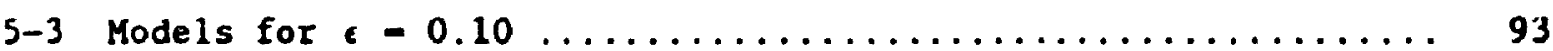

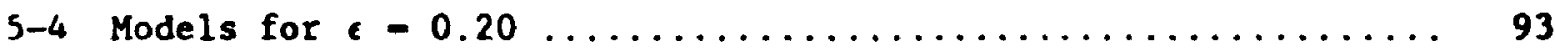

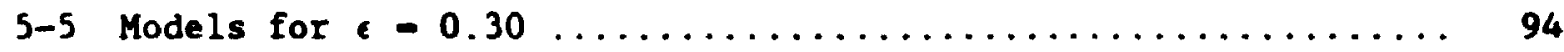




\section{LIST OF FIGURES}

Figure

Description

Page

2-1 Beta Law Velocity Distributions $\ldots \ldots \ldots \ldots \ldots \ldots \ldots \ldots \ldots \ldots$

2-2 Dependence of the Force Terms on $r / R$ for $\beta-20 \ldots \ldots \ldots \ldots . \ldots 22$

2-3 Dependence of $F_{x} / F_{g}$ on $r / R$ for $\beta-1,5,10$ and $20 \ldots \ldots \ldots \ldots$

2-4 Influence of a Corona at the Base of wind on $F_{x} \ldots \ldots \ldots \ldots$

2-5 Dependence of the Force Terms on $r / R$ for a Keplerian Disk ... 26

2-6 Power Law Velocity Distributions $\ldots \ldots \ldots \ldots \ldots \ldots \ldots \ldots \ldots$

2-7 Dependence of the Force Terms on $r / R$ for $m-1 \ldots \ldots \ldots \ldots$

2-8 Dependence of the Force Terms on $r / R$ for $r$ Cas $\ldots \ldots \ldots \ldots . \ldots 33$

2-9 Dependence of the Force Terms on $r / R$ for $\beta$ Mon ......... 34

2-10 Dependence of the Force Terms on $r / R$ for EW Lac ........ 35

2-11 Dependence of the Force Terms on $r / R$ for $\psi$ Per $\ldots \ldots \ldots \ldots \ldots$

2-12 Dependence of the Force Terms on $r / R$ for $\eta$ Tau ........ 37

2-13 Defendence of the Force Terms on $r / R$ for $\beta$ CMi $\ldots \ldots \ldots \ldots \ldots 38$

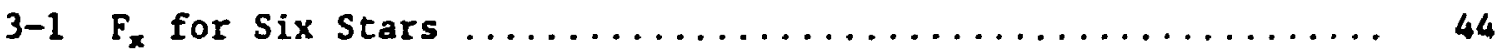

3-2 Normalized $F_{x}$ for $s i x$ stars $\ldots \ldots \ldots \ldots \ldots \ldots \ldots \ldots \ldots \ldots \ldots$

4-1 Azimuthal Velocity for $\beta$ Laws $\ldots \ldots \ldots \ldots \ldots \ldots \ldots \ldots \ldots \ldots$

4-2 Azimuthal Component of the Magnetic Field for $\beta$ Laws ...... 60

4-3 :ransfer Process of Angular Momentum for $\beta$ Laws ......... 61

4-4 Azimuthal Velocity for Models in Table 4-2 $\ldots \ldots \ldots \ldots \ldots \ldots 6$

4-5 Azimuthal Magnetic Field Component for Models in Table 4-2 .. 67

4-6 Azimuthal Velocity for Models in Table 4-3 $\ldots \ldots \ldots \ldots \ldots \ldots 68$

4-7 Azimuthal Magnetic Field Component for Models in Table 4-3 .. 69

4-8 Azimuthal Velocity for Models in Table $4-4 \ldots \ldots \ldots \ldots \ldots \ldots$ 71

4-9 Azimuthal Magnetic Field Component for Models in Table 4-4 .. 72

4-10 Radial Component of the Magnetic Force for Models 15 and 16,74 
4-11 The Dependence of the Force Terms on $r / R$ for Model $15 \ldots 75$

4-12 The Dependence of the Force Terms on $r / R$ for Model $16 \ldots 77$

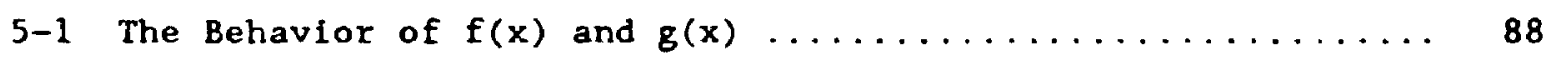

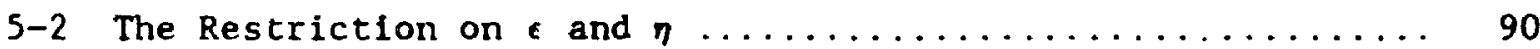

5-3 The Radial Velocity Distributions for $\epsilon=0.01 \ldots \ldots \ldots$

5-4 The Radial Velocity Distributions for $\epsilon=0.05 \ldots \ldots \ldots . \ldots 9$

5-5 The Radial Velocity Distributions for $\epsilon=0.10 \ldots \ldots \ldots$

5-6 The Radial Velocity Distributions for $\epsilon=0.20 \ldots \ldots \ldots 9$

5-7 The Radial Velocity Distributions for $\epsilon=0.30 \ldots \ldots$

5-8 The Comparison of Velocity Distributions $\ldots \ldots \ldots \ldots \ldots 2$

5-9 A Calculated $\mathrm{H \alpha}$ Profile ......................... 104

5-10 The Cross Section of a Be star Envelope ............ 105 


\section{CLIAPTER 1 INTRODUCTION}

A Be star is defined as an early-type star with luminosity class ranging from $V$ to III, whose spectrum shows, or has shown at some time, hydrogen Balmer lines in emission. They were first discovered by Father Angelo Seccht (1866) in Rome 120 years ago. When he visually inspected spectra of $\gamma$ Cas and $\beta$ Lyr, he found that the $\mathrm{H} \beta$ lines were in emission in the spectra. Since then hundreds of Be stars have been discovered. The catalogue of Merrill and Burwell (1950) contains 1088 Be stars; Wackerling (1970) compiled a catalogue containing nearly 3000 Be stars. The Be stars represent approximately 208 of B type stars, with a maximum frequency at B2-B3. The frequency of occurrence drops rapidly towards late 0 type stars and gradually towards early A type stars. Since Be stars were discovered, people have devoted a tremendous effort, both observationally and theoretically, to try to understand them. In this chapter, I will briefly review observations and models of Be stars. Good general reviews on Be stars can be found in I.A.U. Symposiums No. 70 (Slettebak 1976), and No. 98 (Jaschek 1982), I.A.U. Colloquium No. 92 (Slettebak \& Snow 1987) and the book B Stars With and Without Emission Lines by Underhill \& Doazan (1982).

\subsection{Observations}

\subsubsection{Emission Lines}

The most striking characteristic of the Be star phenomenon is the existence of emission lines in the Balmer series. The hydrogen emission lines are often accompanied by emission lines of singly lonized metals, mainly FeII. In general, the emission intensity of the Balmer Ifnes shows a Balmer decrement, 1.e., it is greatest at ha and decreases in strength 
at $\mathrm{H} \beta, \mathrm{Hr}$, and so on up the Balmer series. For some stars emission is only visible at Ha.

The emission may appear as a single peak or as double peaks with a central reversal. When double emission peaks are present, the redward peak and the blueward peak are referred to as $R$ and $V$ components, respectively. The ratio of $V$ to $R, V / R$, is an important parameter to describe the asymmetry of emission profiles. When the $v$ component is stronger than $R$, we say $V / R>1$, otherwise, $V / R<1$.

\subsubsection{Polarization}

Another characteristic property of Be stars is their intrinsic linear polarization. The degree of polarization in the continum is of order 1 percent, and generally does not exceed 2 percent. The fact that it is only detected for Be stars but not for normal B type stars suggests that the polarization originates in the envelopes of Be stars. Although the polarization is not high, it provides strong evidence for the asphericity of the envelopes of Be stars.

\subsubsection{Rotational Velocities of Be Stars}

Many observations of rotational velocities of $\mathrm{Be}$ ztars have been carried out. The revised Catalogue of Stellar Rotational Velocities by Uesugi and Fukuda (1982) contains all published values of stellar projected rotational velocities, $v$ sin $i$. There is a systematic difference between rotational velocities of Be stars and those of normal B type stars (Harmanec 1982). Compared with normal B type stars, Be stars as a class are much faster rotators. However, the rotational velocities of Be stars are still below the critical velocity, $v_{\text {exi }}$, which is defined 
as the rotational velocity at which centrifugal force balances gravity, i.e.,

$$
v_{c r i}^{2}-\frac{G M\left(1-\Gamma_{0}\right)}{R}
$$

where $G$ is the constant of gravity: $M$ is the stellar mass: $R$ is the stellar radius; and $\Gamma_{0}$ describes the radiation force due to electron scattering. Using the observations of Slettebak (1982) for all Be stars brighter than $6^{\mathrm{m}}$, Chen \& Huang (1986) concluded that Be stars are rotating at about 0.7 of the critical velocity.

\subsubsection{Infrared Observations}

The first indication that Be stars have infrared excess was found by Johnson and his collaborators (Johnson et al. 1966, Johnson 1967). They observed 85 early type star, in the wavebands $K(2.2 \mu)$ and $L(3.5 \mu)$. All Be stars of these 85 stars were found to have an excess. They concluded that the excess is due to IR emission from circumstellar envelopes. The observations by Gehrz et al. (1974) for 33 Be stars at longer wavelengths (eight wavelengths between 2.3 and $19.5 \mu$ ) provide strong evidence that the IR excess from Be stars is from free-free emission rather than circumstellar dust emission.

The IRAS satellite obtained data at even longer wavelengths, $12 \mu$, $25 \mu, 60 \mu$ and $100 \mu$. Waters, Cote \& Aumann (1987) plotted all stars that are listed in the Bright Star Catalogue and also in the IrAs Point Source Catalogue in color diagrams of $V-[12]$ and $V-[25]$ verses $B-V$ and found that most stars that have an IR excess are Be stars. Waters, Cote \& Lamers (1987) interpreted the IRAS data for Be stars in terms of a disk-like model and derived the velocity distribution in the disk. They found that 
$V-V_{0}(r / R)$, with $0<m<1.5$, where $V_{0}$, the wind speed at the surface of the star of radius $R$, was assumed to be $5 \mathrm{~km} / \mathrm{s}$. The expansion speed in their disk models is typically less than $200 \mathrm{~km} / \mathrm{s}$ within 20 stellar radii.

\subsubsection{X-ray Observations}

Very little information has been collected about soft $\mathrm{X}$-ray emission, which is thought to be a characteristic of stellar coronae (van den Heuvel and Rapport 1987). The only Be star for which soft x-ray emission has been detected is 5 Oph (Cassinelii 1985), with a x-ray luminosity $\mathrm{L}_{x}-10^{-6} \mathrm{~L}_{\text {optical }}$. This relation between $\mathrm{L}_{x}$ and $\mathrm{L}_{\text {optical }}$ is a general relation for 0 type stars.

Hard $x$-ray emission has also been detected for some 20 Be stars. These $20 x$-ray emission sources are found to be in binary systems with a Be star as the primary star and a compact star as the secondary star (van den Heuvel and Rappaport 1987). From studies of Be/x-ray binaries, Waters et al. (1988) found that the velocity of the wind relative to the neutron star at the neutron star's position was in the range 150 and $600 \mathrm{~km} / \mathrm{s}$, but more commonly between 150 and $300 \mathrm{~km} / \mathrm{s}$. These velocities occur at distances between about 10 and 40 stellar radii from the Be star.

\subsubsection{Variability and Timescales}

The variability of Be stars is well known. Virtually all Be stars show variations of one kind or another. As far as the optical region is concerned, the Balmer emission strengths change and may even disappear completely; the emission profiles may also change. Be stars also experience variations in other regions such as ultraviolet region. IUE observations indicate that UV lines experience strong variation. 
The timescales of these varlations range from days to years. Delplace \& Hubert (1975) have demonstrated that the timescale for emission variation varies from over 50 year for B8 stars to 8 years for B2 stars. Timescales of days or even hours for emission variations have been reported (Hutchings 1970, Doazan 1976, Bahng 1976).

\subsection{Be Star Models}

The first dynamical model for Be stars was proposed by struve (1931). Since then many other models have been suggested (see Poeckert 1982 and Marlborough 1987 for details). Now people generally belleve that the emission lines arise from the envelopes surrounding the Be stars. Constructing a Be star model really contains two aspects: 1) the dynamical problem, i.e., how the envelopes are formed; and 2) the radiation transfer problem, i.e., how the emission lines are formed in the envelopes. The two aspects of the problem are really closely related. Loosely speaking, to solve the radiation transfer problem one needs the dynamical structure of the envelope, i.e., a velocity distribution and a density distribution, as the input; and to test the dynamical solution, one needs to solve the radiation transfer problem to see if the correct emission profile, polarization, infrared excess etc. can be produced. More precisely, to construct a Be star model one really has to solve both the dynamical and radiation transfer problems simultaneously to obtain the correct solution. However, it is still generally the case that investigators only work on one of these two aspects at one time, partly because of the complexit; of the problem and partly because of lack of knowledge of the dominant physical processes around Be stars. Since in my thesis I shall focus on the dynamical aspect of the problem, I shall review mainly the dynamical 
aspect of the models rather than radiation transfer problem.

\subsubsection{Basic Equations}

The basic equations to describe the dynamical structure of the envelopes were summarized by Marlborough (1976) more than fifteen years ago and are still relevant today. They are:

$$
\begin{gathered}
\frac{\partial \rho}{\partial t}+\nabla \cdot \rho V-0, \\
\frac{\partial \vec{V}}{\partial t}+\vec{V} \cdot \nabla \vec{V}--\frac{1}{\rho} \nabla P_{g}+\vec{F}+E \\
\frac{\partial E_{g}}{\partial t}+\vec{V} \cdot \nabla E_{g}+P_{g} \frac{\partial}{\partial t}\left(\frac{1}{\rho}\right)+P_{g} \vec{V} \cdot \nabla\left(\frac{1}{\rho}\right)-G-L .
\end{gathered}
$$

Equations $(1-2 a),(1-2 b)$ and $(1-2 c)$ are, respectively, the equation of continuity, the equation of motion and the equation of conservation of energy. In the equations, $\vec{V}$ is the velocity; $P_{g}$ is the gas pressure; $\vec{F}$ is the total body force per unit mass, which includes gravitation due to the central star, the radiation force, the Lorentz force if a magnetic field is present, etc.; $f$ is the total boundary force per unit mass, which includes viscous effect; $E_{g}$ is the internal energy of the gas per unit mass; $G$ is the rate of energy gain per unit mass of the gas; and finally $L$ is the rate of energy loss per unit mass of the gas.

Equations $(1-2 a),(1-2 b)$ and $(1-2 c)$ are a set of five partial differential equations. In a general case, we are far away from solving these equations simultaneously. Major simplifications have to be made to make progress to solve the equations.

\subsubsection{Struve's Rotational Model}


Struve (1931) proposed his rotational model sixty years ago. In his model, he tried to solve two aspects of the problems, 1.e., a) in which region the emission lines are formed, and b) how this region is formed.

He proposed that the emission lines were formed in an extended envelope, which is concentrated towards the equatorial plane; and the extended envelope was ejected from the equator region of the star, which was assumed to be rotating at the critical rotation speed. As I discussed

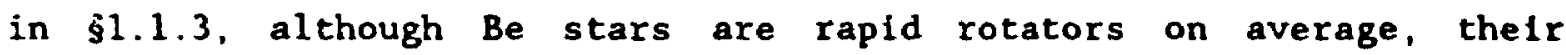
rotational velocities are still below critical velocities. Therefore, although rotation may play an important role in the formation of a Be star envelope, rotation by itself is still not sufficient to drive materlal out to form the envelope. There must be some other mechanism(s) which 1 s/are important.

\subsubsection{The PM Model}

For a Be star envelope with an assumed velocity distribution and surface density, Poeckert \& Marlborough (1978, hereafter PM) calculated the Ha profile, the Balmer decrement, the linear polarfzation, and the continuous energy distribution in the optical and near infrared regions. By comparing calculated Ha profiles and continuum polarization with observations, they determined the velocity distribution and the surface density. The data used to construct this model were obtained during the period 1973-1977. The Ha profile used in their model was of the shape $V / R$ $<1$.

The PM model was able to explain the Ha profile, the Balmer decrement, the linear polarization, and the continuous energy distribution in the optical and near infrared regions. Although the PM model is an ad- 
hoc model in the sense that it only solves ure radiatiun transfer problem for an assumed dynamical structure, it does provide some constraints on the dynamical structure of the region in which Ha emission, linear polarization and emission at continuous region are formed. To explain the Ha profile with the PM model, the envelope has to be slowly expanding. The radial velocity of the disk material could not exceed $150 \mathrm{~km} / \mathrm{s}$ within 20 stellar radii. In fact, the radial velocity distribution used in PM model can be represented by a $\beta$ law distribution, $V(r)-V_{0}+\left(V_{0}-V_{0}\right)(1-$ $\mathrm{R} / \mathrm{r})^{\circ}$, with $\mathrm{V}_{0}-7.5 \mathrm{~km} \mathrm{~s}^{-1}, \mathrm{~V}_{0}-253 \mathrm{~km} \mathrm{~g}^{-1}$ and $\beta-14.5$. The radial velocity is about $60 \mathrm{~km} \mathrm{~s} \mathrm{~s}^{-1}$ at 10 stellar radii.

1.2.4 A Radiation Driven Wind Model due to Optically Thick Lines

The radiation driven wind model due to strong lines was first developed for and successfully applied to 0 type stars (Lucy \& Solomon 1970. Castor, Abbott \& Klein 1975, hereafter CAK). A recent comprehensive review of radiation driven winds is given by Kudritzki (1988).

In general, the radiation driven model due to strong lines is able to explain well the UV observations of these hot stars. Since B stars, especially those of early spectral type, have a strong radiation field, it is reasonable to suspect that similar radiation forces may be an important contributor to the wind dynamics of Be stars. For example, the UV spectra of 5 Oph, $09.5 \mathrm{~V}$ (Morton 1976) and $r$ Sco, BO V (Lamers \& Rogerson 1978) show that the winds from these stars are accelerated to the speeds $>1000$ $\mathrm{km} / \mathrm{s}$. Such high terminal velocities are taken as direct evidence of a radiation driven wind in these and similer stars (see CAK). Thus Be stars with spectral types hotter than about $B 1-2$ are expected to have a radiation driven wind in which strong lines are a major contributor to the 
radiation force (Abbott 1979). For Be stars cooler than B1-2 the radiation force from strong lines may also be expected to have some influence on the dynamics of the circumstellar material.

However, one of the distinctive characteristics of a wind driven by strong lines is a rapid acceleration to the terminal speed over a distance of about one stellar radius. And the terminal speed itself is of order the escape speed at the surface of the star. For main sequence B stars, this means a wind speed of order $1000 \mathrm{~km} / \mathrm{s}$ within one stellar radius of the surface. Two problems arise immediately from the magnitude of this speed. First, according to the PM model, the region of the circumstellar envelope within 10 stellar radil of the surface makes a significant contribution to the shape of the Ha emission line profile, which is not grossly asymmetric about line center. If the radial component of velacity were of order $1000 \mathrm{~km} / \mathrm{s}$ within this region, one would expect a P Cygni profile for the Ha emission line. The Ha emission line proflles in Be stars are normally neither type I nor type II P Cygni profiles (Beals 1951). In fact, in the visible region one does not see any evidence of a rapid expansion of Be star envelopes. Secondly, a velocity increase from some fraction of the sound speed at the surface to $-1000 \mathrm{~km} / \mathrm{s}$ within one stellar radius will produce a density decrease of comparable magnitude from the equation of continuity. Such a decrease is inconsistent with the density distributions derived from IRAS data by Waters (1986), and Waters et al. (1987). It is also unlikely that an envelope with such rapld density decrease will yield an Ha emission profile of the required shape and strength, although this has not yet been investigated in detail.

To take into account the influence of rotation, Marlborough Zamir (1984) considered the CAK wind solutions along the rotation axis and in 
the equatorial plane of a rotating star. In their model, the star was treated as a point source of radiation and the wind was assumed to have a temperature less than the stellar temperature. They found that the equatorial solution would have a much lower velocity and consequently much higher density than the solution along the polar axis. The resulting wind had some similarity to the ad hoc PM model. Marlborough (1987) proposed such a rotationally distorted wind model for Be stars in a paper presented in 1986. However, at the same time, Friend \& Abbott (1986) found that an extended disk-like region of high density and low velocity in the equatorial plane did not occur when the finite size of the star was taken into account, even for a star rotating at 70-80 of the critical speed. Instead they found a wind which again accelerates rapidly very similar to the CAK result, reaching half the terminal speed at about 1.6 stellar radii from the surface. The predicted terminal speed is even higher when the finite angular size of the star is included. Unpublished results by Marlborough for a BO V star, rotating at 70 of the critical speed, predict a wind speed of $-1000 \mathrm{~km} / \mathrm{s}$ at 1.5 stellar radii in the equatorial plane when the finite size of the star is included.

Poe \& Fiend (1986) included a magnetic field to a radiation driven wind, In which strong lines are a major contributor. They found that the presence of a magnetic field does not change the velocity structure dramatically. Wind speeds of order $1000 \mathrm{~km} / \mathrm{s}$ are again reached within 1-2 stellar radil.

The magnitude of the observed linear polarization in Be stars may also pose a problem for a radiation driven wind dominated by strong 11 nes. To produce the observed magnitude of polarization the distribution of scatterers must not be spherically symmetric. However a radiation driven 
wind model will produce a spherically symmetric wind unless rotation is included. A radiation driven wind from a rotating star is expected to have a different density structure in the equatorial plane compared to that along the rotation axis because of the increased mass loss rate in the equatorial plane (Friend \& Abbott 1986). It remains to be seen whether such a matter distribution can produce linear polarization of the correct magnitude.

The above discussions suggest that the radiation force with strong lines as a major contributor is unlikely to be the force dominating the dynamics of matter in the equatorial regions of the envelopes of Be stars. Besides, Abbott $(1979,1985)$ has shown that radiation driven winds due to strong lines are self-initiating in stars brighter than $M_{b o l}-6$. This 1imit corresponds to spectral type B2 for main sequence stars. Therefore, even if the radiation driven wind model due to strong lines is able to explain the formation of envelopes of Be stars hotter than B2, some other mechanism is still needed to provide the initial acceleration for Be stars cooler than B2.

\subsubsection{The Thomas and Doazan Model}

Thomas and Doazan (Doazan \& Thomas 1982, Doazan 1987) assume that the envelopes of Be stars have a spherical shape distinguished by different radial zones. The radial sequence of the zones of the envelope is as follows: 1) a quasi-thermal photosphere, in which the outflow is small, $v \leq q / 3$, where $v$ is the outflow velocity and $q$ the one-dimenslonal thermal velocity; 2) a chromosphere, in which a non-radiative energy flux dissipates sufficient energy to raise the temperature significantly; 3) a corona, in which the gas is accelerated to high speed and the temperature 
is raised to over a million degrees so that superionized lons could form;

4) a post-corona region, in which the gas begins to cool but continues to be accelerated; and 5) a low-velocity, cool and extended region, in which Ha emission is formed. The purpose of introducing such a complicated envelope is try to explain the observations from all spectral regions. But since no detailed calculation has ever been done, it is too early to be optimistic about this model.

\section{2 .6 Summary}

Although all Be models claim to account successfuily for some aspects of Be star behaviour, each of them has at least one or two serious faults. First each model is ad t.uc, and second none can explain all the observed properties of Be stars and their circumstellar envelopes. Despite the best efforts made by numerous astronomers, we are still a long way from being able to construct a model of a Be star from first principles. This situation of Be star models reflects directly the lack of knowledge of the relevant physical processes responsible for the Be phenomenon or phenomena. It is therefore very useful to consider what dynamical constraints on the possible physical processes responsible for the Be ihenomenon can be deduced from previous observational and theoretical investigations. 
CHAPTER 2 WHICH FORCE CONTROLS THE DYNAMICS OF

THE ENVELOPES IN THE equatorial plane?

\subsection{INTRODUCTION}

From the brief review of Be stars models in Chapter 1, we can see that no single model can explain Be stars satisfactorily. Even worse, we do not even know what is the driving mechanism which is responsible for the formation of the envelopes. Rotation is inportant but by itself is not sufficient to drive the material out to form the envelope. A radiation force due to optically thick lines could be important, but it cannot be the dominant force controlling the dynamics of the c1rcumstellar matter near the equatorial planes of Be stars. The obvious question then is what is the dominant force? The answer to this question is unfortunately not presently known. In this chapter I hope to provide some information which may assist one to answer this question. Since the nature of this force is unknown, I will refer this force as $F_{x}$. Instead of guessing the form of $F_{x}$. I inverted the equation of motion to solve the radial dependence of $F_{x}$ required to give a velocity distribution, which does not contradict information deduced from ha profiles and infrared data. A similar procedure has been applied by Lamers (1986) in an investigation of the dynamics of the wind of $P$ Cygnt. For this star Lamers concluded that the dynamics of the wind was best explained by radiation forces arising from a large number of optically thin 1 ines.

\subsection{FUNCTIONAL FORMS OF THE VELOCITY LAWS}

Since the form of $F_{x}$ is largely dependent on the choice of the functional form of the radial component of the velocity distribution, one 
has to choose the form of the radial component of the velocity distribution very carefully. The functional forms I used for the radial component of velocity in my investigation correspond to radial velocities which increase with increasing distance from the star. One may Imnediately suspect that models constructed using these velocity laws will only yield ha line profiles with $R>V$. It is true that the PM model did produce an Ha profile with $R>V$. I am unaware of any evidence that such velocity laws can yield models in which Balmer lines have profiles with $\mathrm{V}$ $>R$. But in many cases Ha line profiles in Be stars have $V>R$. In some Be stars cyclic variation of the $V / R$ ratio occurs with phases in which $V$ $>R$ followed by ones in which $R>V$, or vice versa. Since a radial componelit of velocity which increases with increasing distance generally yields profiles with $R>V$, it is common to suspect that profiles with $V$ $>R$ imply an envelope which is contracting, i.e. the radial component of velocity is negative in the emission line forming region.

In order to produce profiles with, respectively, $R>V$ and $V>R$, models in which Be star envelopes first exfanded and then contracted were proposed in the past. Mclaughlin (1961) reviewed these interpretations of $V / R$ cycles and concluded that such simple models were incapable of accounting for the $V / R$ variations in the Be stars he himself had observed over many $V / R$ cycles. Specifically he could not find any evidence of the accumulation of matter in the vicinity of the star in the spectra of most of the stars he studied during the $V>R$ contraction phase. If $V>R$ is interpreted as a result of the contraction of the envelopes, one would expect to see the accumulation of the matter.

IRAS observations also provide some key evidence that the envelopes of Be stars are expanding even for stars with profiles $V>R$. Waters et 
al. (1987) discussed the IRAS data for a large number of Be stars. They found that the IRAS data could be interpreted in terms of a density distribution, $\rho(r)$, for which $p(r) \propto 1 / r^{n}$. For the vast majority of the stars studied, $n>2$; thus the density decreased outward faster than $1 / \mathrm{r}^{2}$. In the following paragraph, we will see that this kind density distribution implies an expanding envelope. Since these stars were observed randomly by IRAS, it is reasonable to expect that some of these Be stars were observed in phases when $V>R$. So Ha line profiles can be observed with $R>V$ or $V>R$ when the envelope is expanding.

There are two possible dynamical models consistent with these results in a steady state situation. First, the star loses mass at a constant rate with velocity given by $v_{0}(r / R)$, where $m-n-2$. If $n>$ 2 as the IRAS data suggest for the bulk of the Be stars observed, then $m$ $>0$, i.e., the velocity increases with increasing distance. Alternately the star could gain mass at a constant rate with velocity given by $-V_{0}(r / R)=$. In this model, material is falling towards the star and the contracting velocity decreases with decreasing distance. However if matter is really falling from a distance of many stellar radil into the gravitational potential well and eventually accreting onto a Be star, one expects the infall velocity initially to increase with decreasing distance, perhaps even becoming supersonic and then becoming subsonic across one or more accretion shocks.

The emission intensity of both the infrared continuum and the Balmer lines depend on the square of the gas denilty. Therefore a signiflcant contribution to both will come from the same volume elements. If the $V / R$ cycles reflect alternately expansion and contraction phases, both ilnes and continum should vary during a $\mathrm{V} / \mathrm{R}$ cycle since the gas density varles. 
Telting et al. (1991) have discussed the long term variabllity of the continum and the Iine spectrum of the Be star $\gamma$ Cas. They assume the gas to be distributed either spherically symmetrically or disk-shaped, in either case with a density distribution varying as $1 / r^{0}$, and use infrared data to compute $n$ as a function of time. The IR colours yield values of n $>2$, with relatively small variation in $n$ over a time interval in which several cycles of $V / R$ variations of $H \beta$ occurred. No correlation between the variation of the $V / R$ ratio and that of the radial density distribution was found.

If the $V / R$ variation is not due to a cycle of alternate expansion and contraction, what causes it? I do not have a definitive answer to this question yet. I do notice, however, that some well-known $V / R$ variables are binaries. The $x$-ray source, 4U1258-61, may help us to understand the $V / R$ variation. This system is thought to be a binary comprising a Be star and a neutron star. The star was detected as an $x$-ray source from discovery in 1969 until 1984 after which no $x$-ray emission was observed. Waters et al. (1988) have shown that the continuous $x$-ray emission in Be/X-ray binaries can be u.aderstood if the neutron star accretes matter from a dense, slowly expanding disk-1ike region around the Be star. Corbet et al. (1986) observed V/R variations of Ho between 1977 and 1981 during the time when the system was a x-ray source. The $x$-ray emission suggests that outflow is continuing in spite of the $V / R$ varlations. Therefore the $V / R$ variations apparently have nothing to do with the outflow behavior in this star.

In addition to continuous $x$-ray emission, periodic $x$-ray flaring has also been detected in this Be/x-ray system with a time scale of about 132 days. This time scale is suggested as being the binary orbital period. 
Corbet et al. (1986) also found that the $V / R$ variation of the Ha emission profile may be modulated at the x-ray flare period of approximately 132 days. This might be additional evidence that $V / R$ variations are not due to expansion and contraction of the envelope but instead to something else.

All these results suggest to me that one should be careful in adopting the expansion/contraction model for the $V / R$ variations. For this reason I consider only velocity laws for which the velocity increases with increasing distance from the star, 1.e. those consistent with the IR continuum data, the data on which I am basing the conclusions in this study.

\subsection{DYNAMICS IN THE EqUatorial PLANE}

I consider a system of spherical polar coordinates $(r, \theta, \phi)$ with $r$ 0 at the origin, $\theta-\pi / 2$ the equatorial plane, and $\phi$ the azimuthal angle measured in the same direction as the star rotates about the $z$ axis. The circumstellar envelope is assumed to be symmetric about the rotation axis $(\theta-0, \pi)$ and the equatorial plane. Instead of considering the dynamics of the circumstellar material in a meridional plane, I restrict my attention to the dynamics in the equatorial plane only. Marlborough $\&$ Zamir (1975) discuss the assumptions necessary in order to do this. In addition, I also assume that the viscosity can be neglected. I also assume that the wind is in a steady state. The steady state assumption restricts applicability of my results to time intervals which are short compared to times over which Be characteristics vary significantly.

Under these assumptions, the equation of motion $(1-1 b)$ can be written in the form 


$$
V \frac{d V}{d r}-F-F_{\mathrm{g}}+F_{\mathrm{p}}+F_{\mathrm{e}}+F_{\mathrm{x}}
$$

where $F$, the radial component of the net force per unit mass on a fluid element, is the magnitude of the vector sum of the relevant forces. In equation (2-1), $F_{g}$, the gravitational force per unit mass reduced for electron scattering. Is given by

$$
F_{0}--\frac{G M\left(1-\Gamma_{0}\right)}{r^{2}}
$$

$F_{p}$, the force per unit mass due to the gas pressure gradient, can be expressed, using the equation of continuity and the perfect gas law, in the form

$$
F_{\mathrm{D}}-\frac{a^{2}}{V} \frac{d V}{d r}+\frac{2 a^{2}}{r}-\frac{d a^{2}}{d r}
$$

and in the form

$$
F_{D}-\frac{a^{2}}{V} \frac{d V}{d r}+\frac{2 a^{2}}{r}
$$

for the isothermal case, where a is the isothermal sound speed; $F_{e}$, the centrifugal force per unit mass, is given by

$$
F_{c}=\frac{v_{b}^{2}}{r}
$$

The form of $F_{c}$ depends on the rotational velocity law. If angular momentum is conserved in the equatorial plane, then

$$
F_{c}-\lambda^{2} \frac{G M(1-\Gamma) R}{r^{3}},
$$


where $R$ is the stellar radius and $\lambda$, the ratio of the equatorial rotation speed of the star to the critical speed, is given by

$$
\lambda^{2}-\frac{v_{\phi}^{2} R}{G M(1-\Gamma)}
$$

If on the other hand the azimuthal velocity component is Keplerian, then $F_{c}$ is given by

$$
F_{e}-\lambda^{2} \frac{G M(1-\Gamma)}{r^{2}}
$$

In the following study I assume $\lambda^{2}-0.50,1$.e. the rotational velocity of the star is assumed to be 0.71 of the critical rotational speed.

In equation $(2-1), F_{x}$ is the additional or unknown force or forces, information about which $I$ am attempting to obtain. Potential candidates for consideration in $F_{x}$ include the radiation force from some combination of thick and thin lines, magnetic fields, energy transfer from acoustic waves, etc. Instead of trying to guess what the appropriate form of $F_{x}$ should be, I shall assume reasonable forms for $V(r)$ and determine the resulting form of $F_{x}(r)$ from equation (2-1).

\subsection{PARAMETER STUDIES}

\subsubsection{The Beta Law}

It is common to represent the radial component of velocity in a stellar wind by

$$
V(r)-V_{0}+\left(V_{-}-V_{0}\right)\left(1-\frac{R}{r}\right)^{\beta}
$$




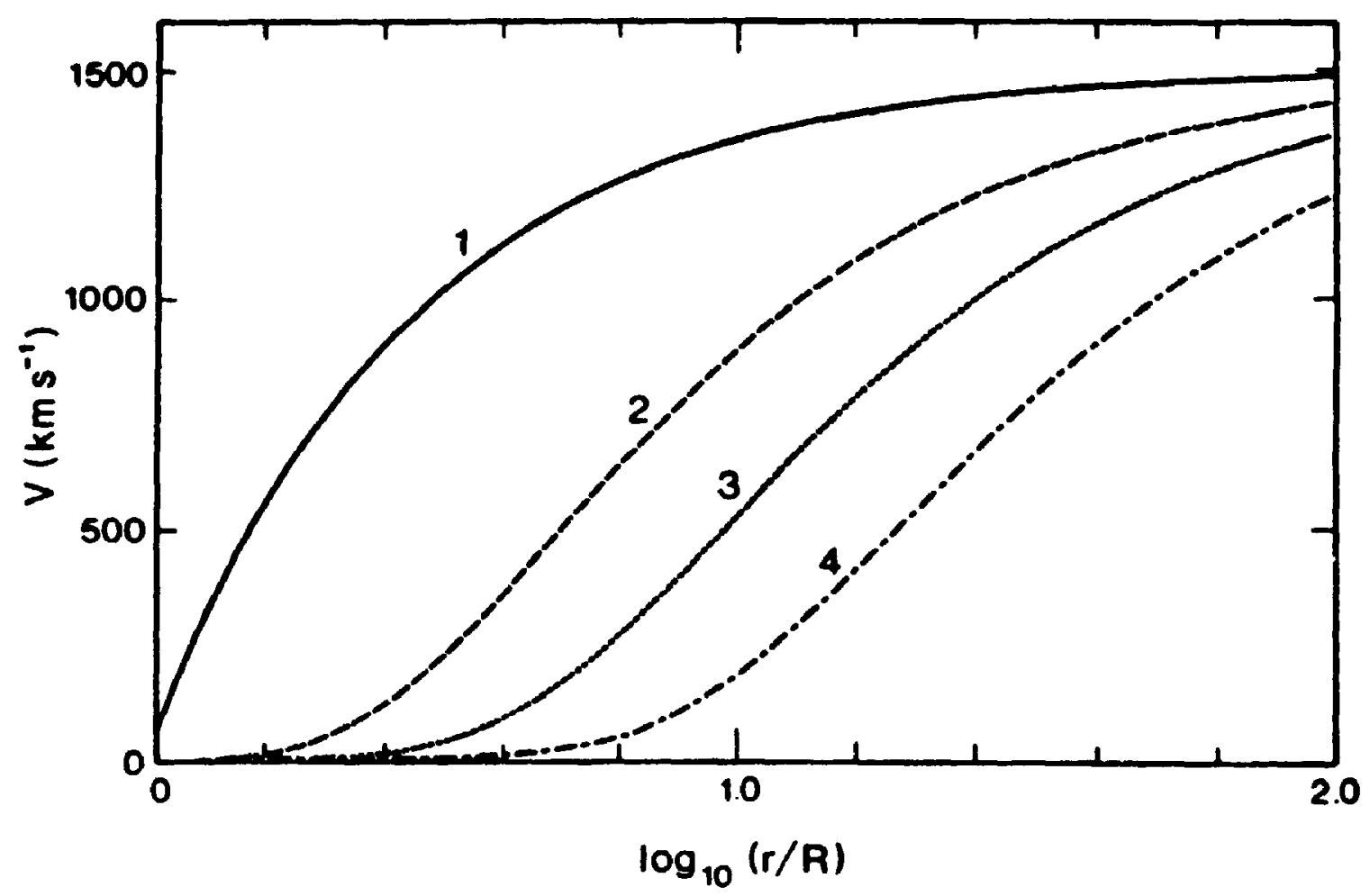

Figure 2-1. The dependence of $V(r)$ on $r / R$ upon the cholce of $\beta$ for beta laws (equ. 2.9) with $V_{0}-0.5$ times the sound speed at $R$ and $V_{0}$ $1500 \mathrm{~km} \mathrm{~s}^{-1}$. Curves labelled $1,2,3$, and 4 correspond respectively to $-1,5,10$, and 20 . 
the so-called Beta Law, where $V_{0}$ is the radial couponent of velocity at the surface, and $v_{0}$ is the terminal speed of the wind. For example the velocity distribution in a CAK wind can be represented fairly well by a small value of $\beta$ of order unity. The velocity distribution of the PM model for $\gamma$ Cas, on the other hand, can be represented by $v_{0}-7.5 \mathrm{~km} / \mathrm{s}$, $V_{\infty}=253 \mathrm{~km} / \mathrm{s}$ and $\beta=14.5$. In Figure 2-1 I show $V(r)$ from equation (9) for four values of $\beta_{i} V_{0}-0.5$ times the sound speed at the surface and $V_{0}$ - $1500 \mathrm{~km} / \mathrm{s}$. The stellar parameters used are those representative of a BO V star: $M=23 M_{0}, R=6.7 R_{0}, T_{\text {aff }}=31,500 \mathrm{~K}, \Gamma-0.044 ;$ I have assumed the wind temperature to be $0.6 \mathrm{~T}_{\text {eff }}$. Note that for $\operatorname{small} \beta, \mathrm{V}(\mathrm{r})$ increases rapidly with $r$, whereas $V(r)$ increases much less rapldly with $r$ for large $\boldsymbol{\beta}$.

\subsubsection{Angular Momentum Conservation}

I have evaluated $F_{x}$ from equation (2-1) for the four velocity distributions with values of $\beta$ given in Figure 2-1, using the values of $v_{0}$ and $V$. given in Figure 2-1 and assuming an isothermal wind which conserves angular momentum in the equatoriai plane. The radial dependence of the various forces in equation (2-1) is qualitatively similar for all values of $\beta$, so I show in Figure 2-2 only the results for $\beta-20$. The pressure term is always two orders of magnitude smaller than $F_{x}$ for all $\beta$. The gravitational and centrifugal terms are the same for all values of $\beta$. Differences in $F_{x}$ reflect directly the differences in the term $\mathrm{VdV} / \mathrm{dr}$. To see how the value of $\beta$ influences $F_{x}$, I show $F_{x}$ normalized in terms of the gravity term, 1.e., $F_{x}(r) / F_{q}(r)$, for the four values of $\beta$ in Figure 2-3. For $\beta=1, F_{x}$ dominates all forces within a short distance from the stellar surface. The $V(r)$ distribution which would result from such a $F_{2}(r)$ 


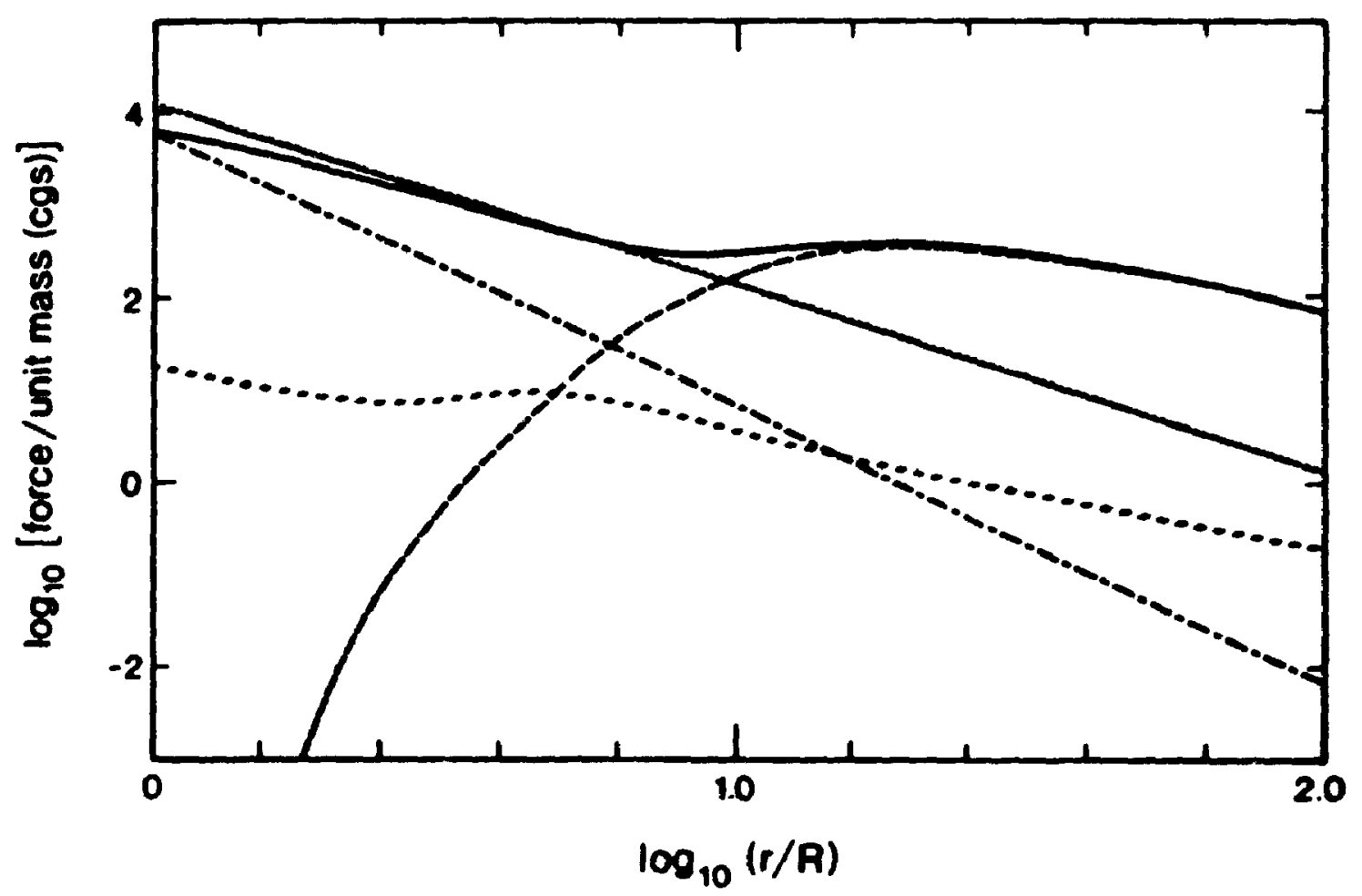

Figure 2-2. The dependence of the force terms on $r / R$ for an isothermal wind under the assumption that angular momentum is conserved. The solid curve is $F_{x}$, the unknown force; the short dashes represent $F_{0}$, the gravitational force; the long dashes repreisent the inertial term Vov/dr; the dot-dash curve is the centrifugal term; and the short dash curve separated by large spaces is the gas pressure term. 


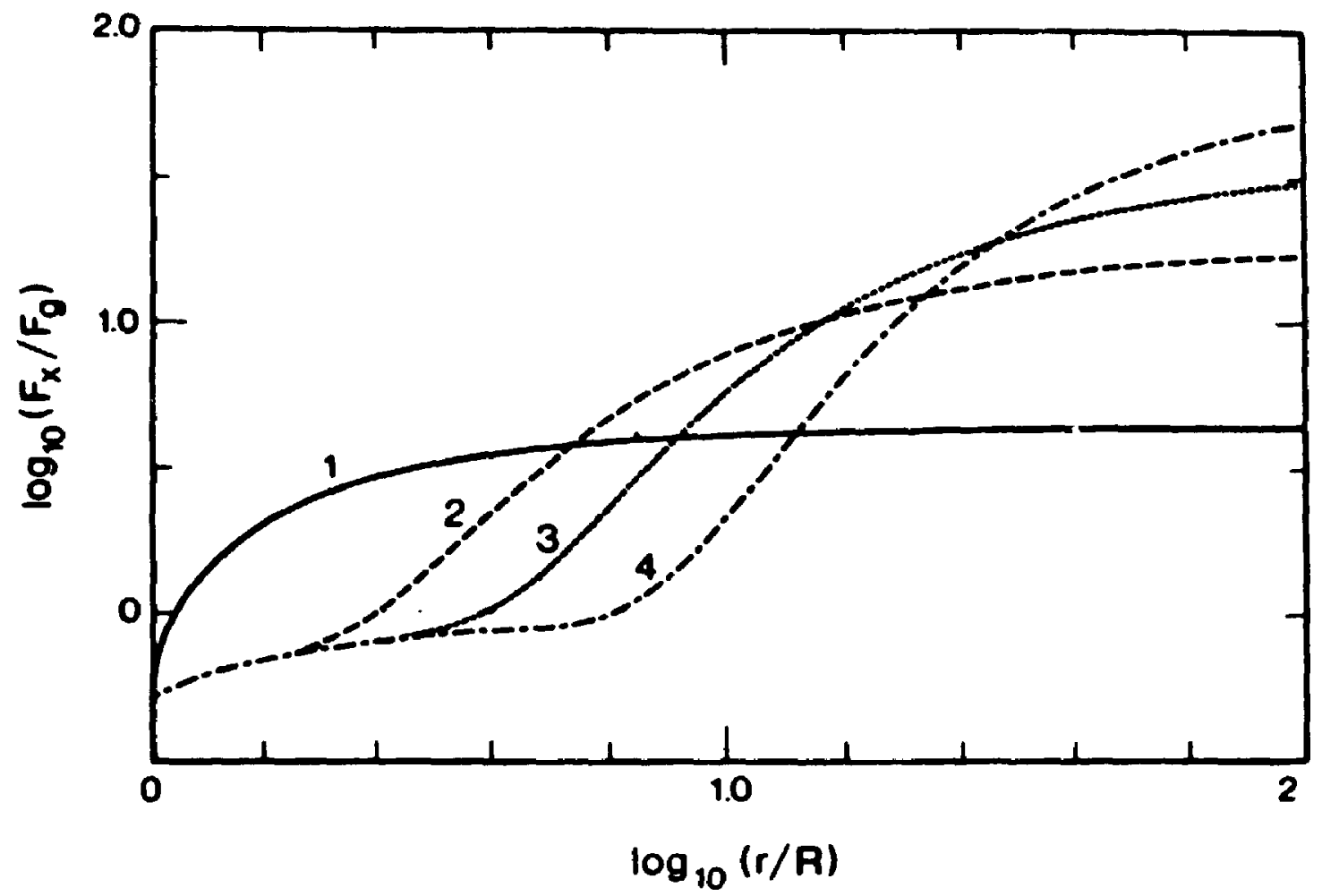

Figure 2-3. The dependence of $F_{2}(r) / F_{3}(r)$ on $r / R$ for different $p$ for the calculations described in the first paragraph of section 4.1.1. Curves labelled $1,2,3$, and 4 correspond respectively to $\beta-1,5,10$, and 20 . 
resembles closely that for a wind driven by strong lines (CAK 1975). As $\beta$ Increases, the distance fron the surface at which $F_{\mathrm{x}}$ first exceeds gravity increases; for $\beta-20, F_{z}(r) / F_{q}(r)>1$ for $r / R>6$.

In the discussion above. I assumed the wind is isothermal. Next I allowed the wind temperature to vary with distance. For cases in which $T(x)$ decreases with increasing $x$, significant differences in $F_{p}$ can occur compared to the isothermal situation. However, because $F_{p} \rightarrow F_{q}, F_{z}$ is essentially unchanged compared to the 1sothermal solution. I then consider how $F_{x}$ would be affected if the wind temperature was larger than the stellar temperature over some interval in the wind. This situation may be relevant because, al though soft $x-r a y$ emission has been detected only for one Be star 5 Oph (Cassinel11 1985), it is possible that some other Be stars also have soft $x$-ray emission which is below current detection limits. A hot corona has been proposed as one explanation for the $x$-ray enission, although there are difficulties with this model (MacFarlane and Cassinel11 1989 and references therein). In order to demonstrate how a region of higher temperature can influence $F_{x}$ compared to the case in which the wind temperature is everywhere as cool as or cooler than the star, I keep all other model parameters constant and assume the temperature distribution in the high temperature region to have a Gaussian shape, increasing from $T_{\text {att }}$ to a maximum of $2 \times 10^{6} \mathrm{~K}$ at $1.5 \mathrm{R}$, decreasing to $T_{\text {otf }}$ at $r-2 R$, and then decreasing as $1 / r^{2}$. The results are shown in Figure 2-4. A comparison of Figures 2-2 and 2-4 shows that the high temperature region affects $F_{x}$ in the particular model studied only near the surface of the star.

\subsubsection{Keplerian Rotation}

So far the rotational velocity is assumed to be determined by angular 


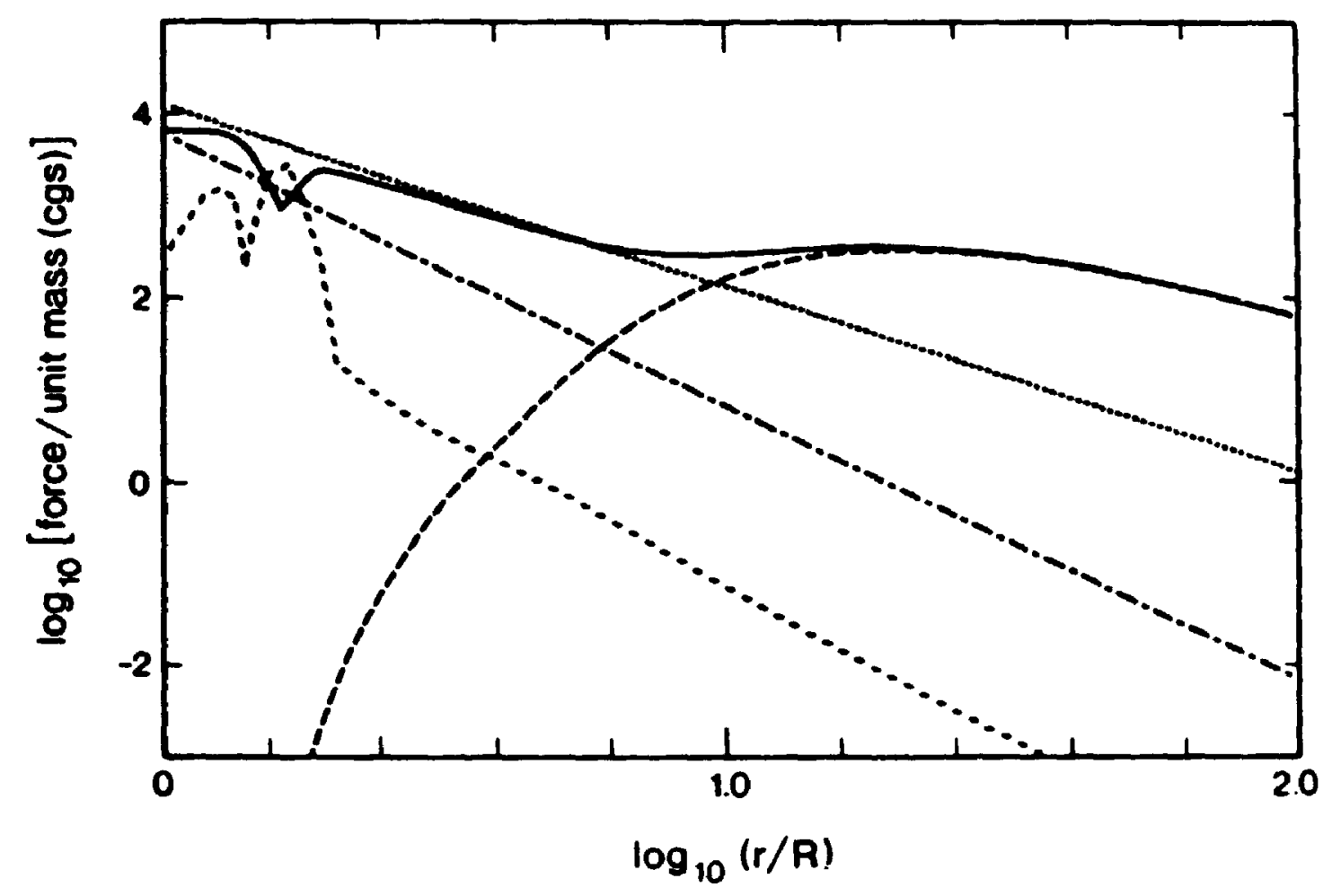

Figure 2.4. The Influence of a corona at the base of the wind on $F_{2}$ for $B-20$ and $V_{0}$ and $V_{0}$ as in Figure 1 . The corona has a saxigur temperature of $2 \times 10^{\circ} \mathrm{K}$ et $\mathrm{r} / \mathrm{R}-1.5$. See the caption of Figure 2 for the Identification of the various force terms. 


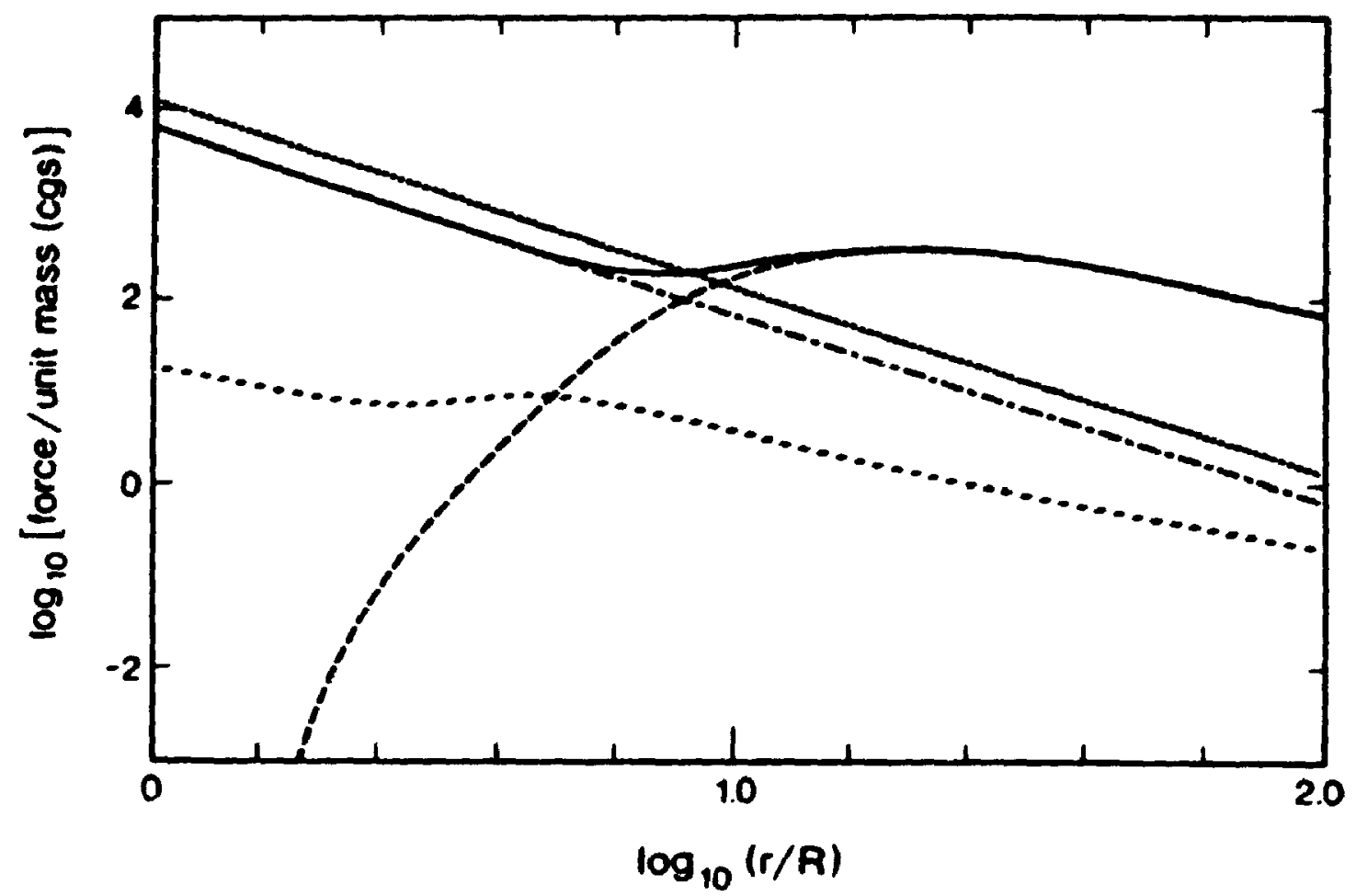

Figure 2.5. The dependence of the force terms on $r / R$ for an isotherwal wind, with $B, V_{0}$, and $v_{0}$ as in Figure 2-2, under the assumption of Keplerian rotation. See the caption of figure 2.2 for the Identification of the varlous force cerms. 
momentum conservation. I have also Investigated one case in which the rotational velocity is assumed to be Keplerian. Limber Marlborough (1968) suggested that the Keplerian rotation could posslbly support Be star envelopes. The results are shown in Figure 2-5. A comparison of Figures 2-2 and 2-5 shows, as expected, that $F_{x}$ is smaller close to the star in the Keplerian case, because the centrifugal force decreases less rapidly with increasing distance compared to the solution in which angular momentum is conserved. Beyond about $16 \mathrm{R}$, the two solutions for $F_{x}$ become indistinguishable, since the contribution from centrifugal force for either case is much smaller than the term $\mathrm{VdV} / \mathrm{dr}$.

\subsubsection{The Power Law}

Waters et al. (1987) have interpreted IRAS data for Be stars using a disk model in which the density $\rho(r)$ is assumed to vary as $r^{2}$ and the opening angle of the disk is assumed to be a constant of $r$. Using the equation of continuity, one will have

$$
V(r)-V_{0}\left(\frac{r}{R}\right)=
$$

a power-law dependence with $m-n-2$. For the stars investigated by Waters et al., $0<m<1.5$. In reality, the opening angle may vary with increasing $x$. This variation in the opening angle will influence the density distribution derived from the continum energy distribution. But one can show that it will not influence the velocity distribution equation (2-10) dramatically.

In Figure 2-6 I show $V(r)$ for three values of $m$; in each case $V_{0}$ is taken to be 0.5 times the sound speed at the surface. For a power-law velocity distribution, the smaller is the power Index $m$, the more siuwly 


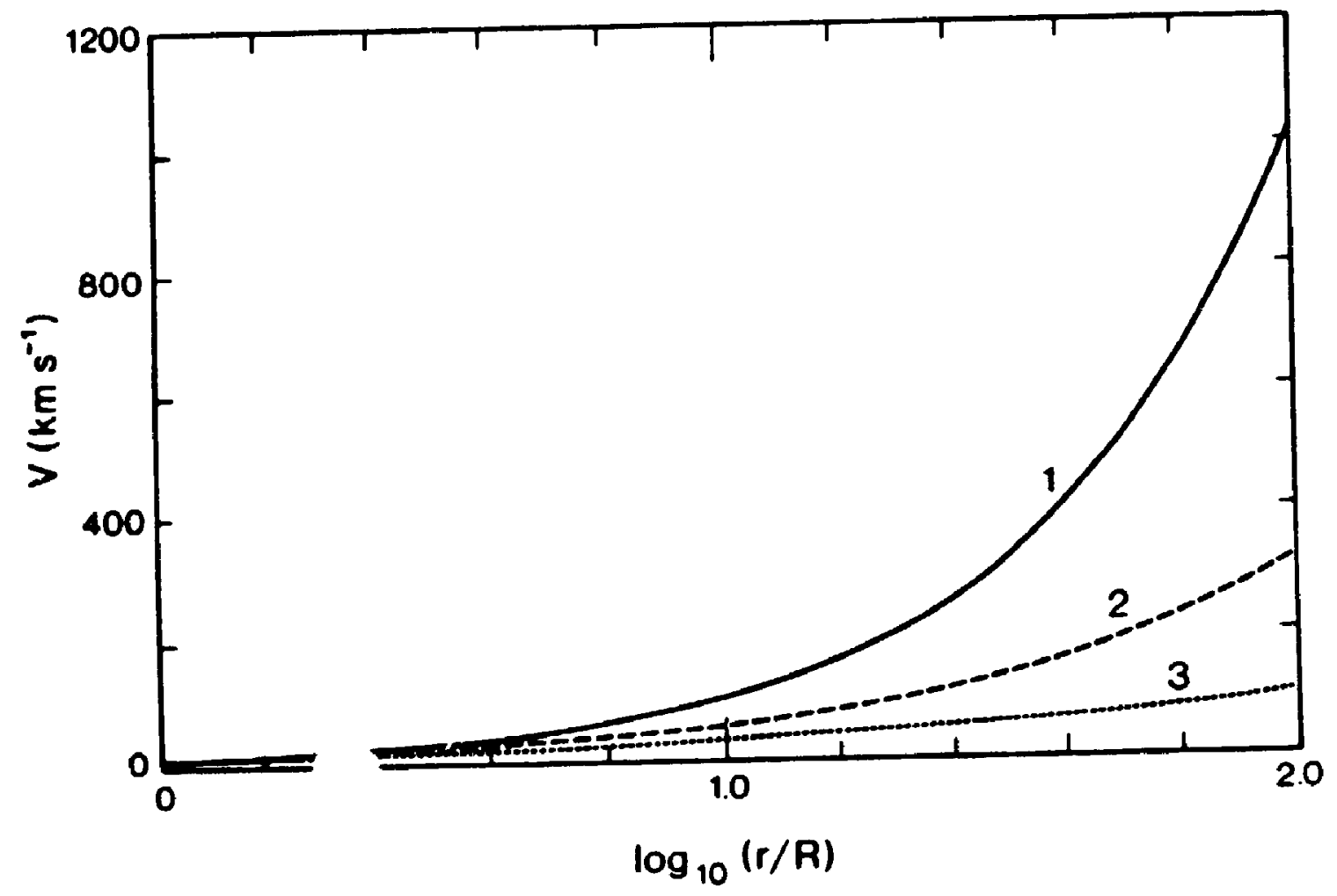

Figure 2-6. The dependence of $V(r)$ on $r / R$ for a pover law velocicy (equ. 10) with $V_{0}=0.5$ times the sound speed at $R$. The curves labelled 1. 2, asd 3 correspond respectively to the power las Index $w-1$. 0.75 , and 0.5 . 


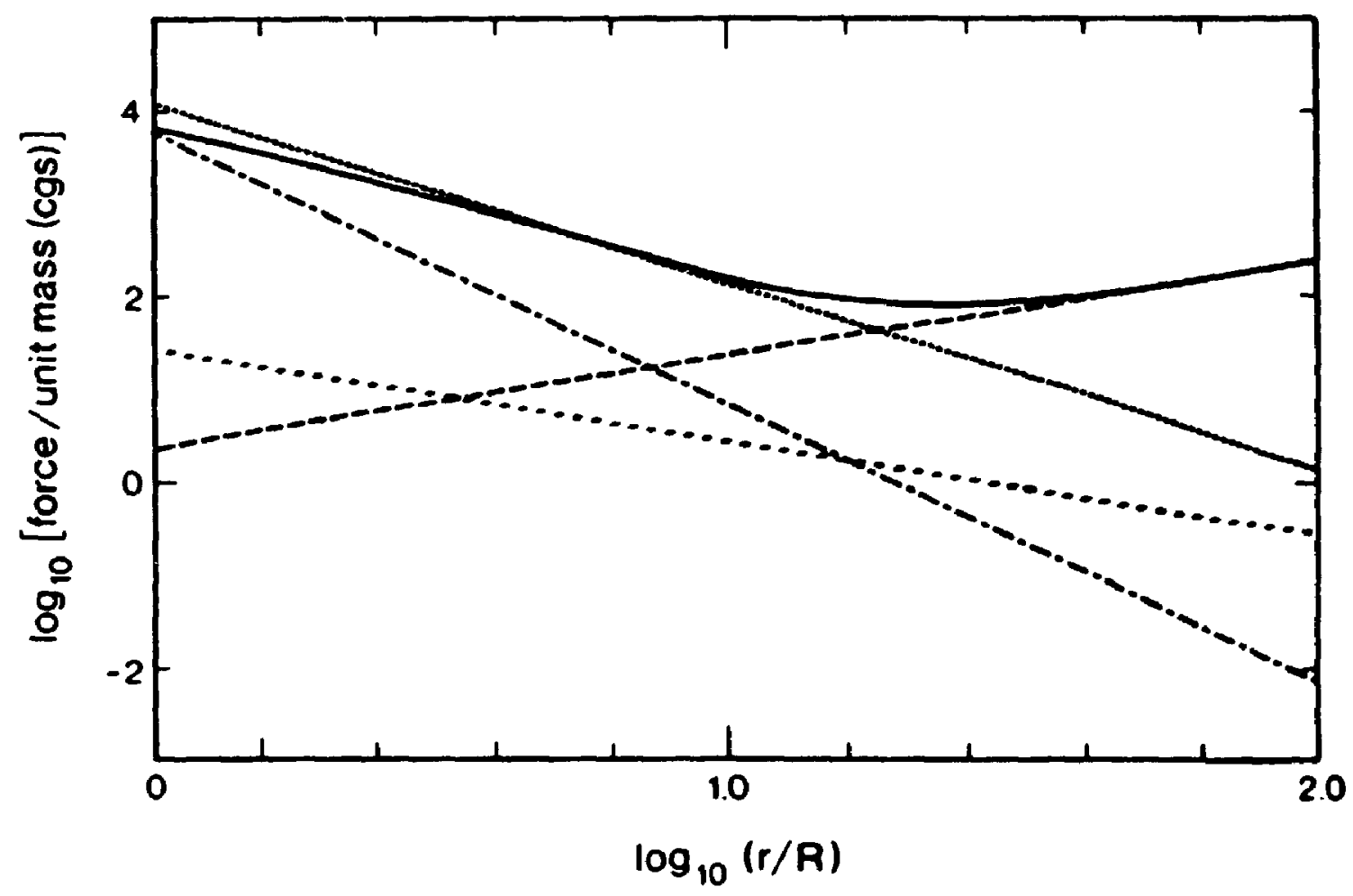

Figure 2.7. The dependence of the force terms on $I / R$ for an isothermal wind with power law index -1 and $V_{0}-0.5$ times the sound speed at $R$, under the assumption that angular momentum is conserved. See the caption of figure $2-2$ for the identification of the varlous force terns. 
TABLE 2-1

Struse Paverotos

\begin{tabular}{|c|c|c|c|c|c|c|}
\hline Star & Sp. Type & $T_{\text {ott }}$ & $M / \mathbb{H}_{0}$ & $R / R_{0}$ & $L / h_{0}$ & - \\
\hline y Cas & Bo.s IVe & 25000 & 16. & 10. & $5.4 \times 10^{4}$ & 1.3 \\
\hline$B$ Mon $A$ & B3 Ve-sheIl & 20000 & 7.5 & 3.5 & $1.9 \times 10^{3}$ & 0.7 \\
\hline EW Lac & B4 III-shell & 16000 & 8.5 & 6. & $3.5 \times 103$ & 0.5 \\
\hline$\downarrow$ Per & B5 IIle-shell & 14500 & 7. & 6. & $1.8 \times 10^{3}$ & 0.7 \\
\hline$\eta$ Tau & B) IIIe & 12000 & 5.5 & 4.5 & $7.0 \times 10^{2}$ & 0.9 \\
\hline B CMI & B8 Ve & 11500 & 3.5 & 2.5 & $1.8 \times 10^{2}$ & 0.9 \\
\hline
\end{tabular}


does the velocity increase. Using the same procedure. I can evaluate $F_{\text {a }}$ corresponding to a power law velocily distribution. In Figure 2-7 I show the radial dependence of the various forces in equation (2-1) with a - 1 . assuming an isothermal wind with angular momentum conservation in the equatorial plane. Note that $F_{\mathrm{z}}$ is less than $F_{\mathrm{g}}$ for $r<10 \mathrm{R}$. For swaller values of $m, F_{x}<F_{q}$ to even larger values of $r$.

\subsection{APPLICATIONS TO SIX Be STARS}

I have applied the methed described above to six well studied Be stars. The parameters of the six star: are given in Table $2-1$. The names of the stars are given in column 1 and tise spectral types in column 2. I put "shell" after the spectral type when the star is classified as a shell star (Slettebak 1982). Columns 3, 4, 5 and 6 give, respectively, the effective temperature, mass, radius and luminosity of the stars.

Waters et al. (1991) have used IR, millimeter and radlo observations of six Be stars (ste Table 2-1) to derive the radial denslty structure of the circumstellar material. They assumed a cone-shaped disc with a power-law density distribution $\left(r^{2}\right)$ and a finite outer radius $R_{d i e c}$. The slope of the IR to radio continum then gives the radial density gradient. Under the assumption of mass continulty, the radial density distribution can be converted to a velocity distribution, which can be used to derive $F_{x}(r)$. I have determined the index in In equation (2-10) from the power law index $n$, given in Table 4 of Waters et al. (1991). Column 7 in Table 2-1 gives the velocity index $m_{0}$. It was found from the analysis that for at least one star ( $\gamma$ Cas) the IR to radio spectrun could not be described with a single value of the density gradient (parameter $n$ ), but that from a certain distance outwards, the density gradient of the lonized material 
Increases significantly. For the other stars, one value of $n$ could describe the IR-radio continuum satisfactorily if the disc has a finite outer radius of the order of 25 to $100 \mathrm{R}$. This in fact means that also for these stars an increase in the density gradient occurs at some distance, but the available data do not allow an accurate determination of the change in density gradient.

I have computed $F_{x}(r)$ for these six stars using the procedure described above. In all cases, I assumed the disk temperature was isothermal and equal to 0.6 of $\mathrm{T}_{\text {otf }}$ In Table $2-1$, that the rotational speed of the star was 0.71 of the critical speed, that angular momentum was conserved in the equatorial plane, and that $v_{0}=$ one half the sound speed at the surface. The resulting values of $F_{x}(r)$ are shown in Ftgures 2-8 to 2-13. Waters et al. (1991) also calculated an improved model for $\gamma$ Cas in which the density index $n$ changes at some distance $R_{e}$ from the star. I have also computed $F_{x}(r)$ for this model but do not show it here because apart from a discontinuity at $R_{c}, F_{x}(r)$ is qualitatively similar in the two cases.

\subsection{DISCUSSION}

Based upon the investigations in the previous sections I can now make some general statements regarding the forces acting in the equatorial regions of the envelopes of Be stars. In all cases the pressure term in equation (2-1) is very small, typically of order 18 of the gravitational force. The only exception to this general trend occurred when a corona was added near the stellar surface. For that case the pressure term can have an important influence upon the dynamics in the region of the corona. The centrifugal force always plays an important role close to the 


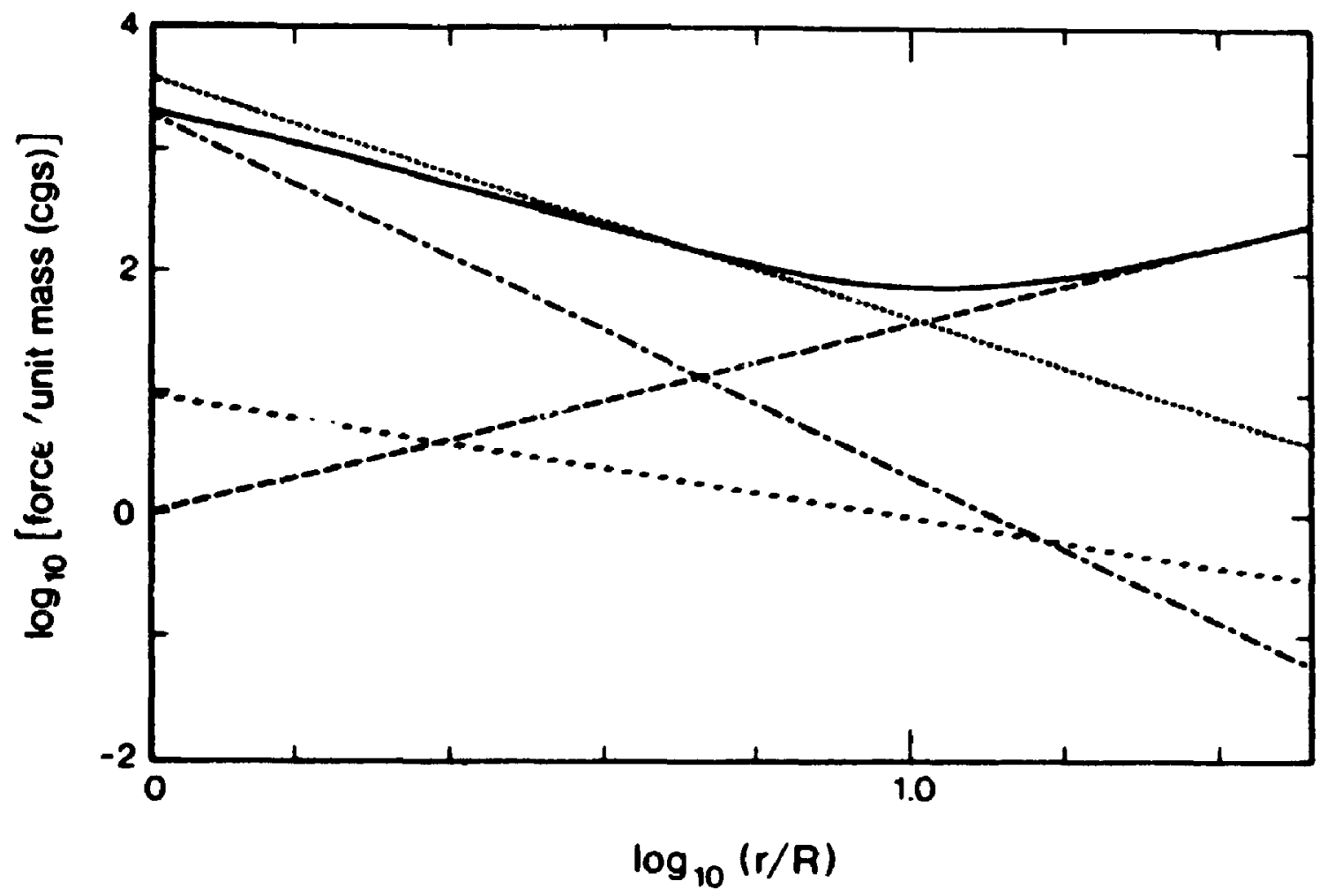

Figure 2.8. The dependence of the force terms on $r / R$ for $y$ Cas with 1.3, where is the power law index in equ. 2-10. The model was assumed to be lsothermal with kinetic temperature 0.6 of $T_{\text {ofse }}$. angular momentur was conserved, and $v_{0}-0.5$ tioes the sound speed at $R$. See the caption of Figure 2.2 for the identification of the various force teres. 


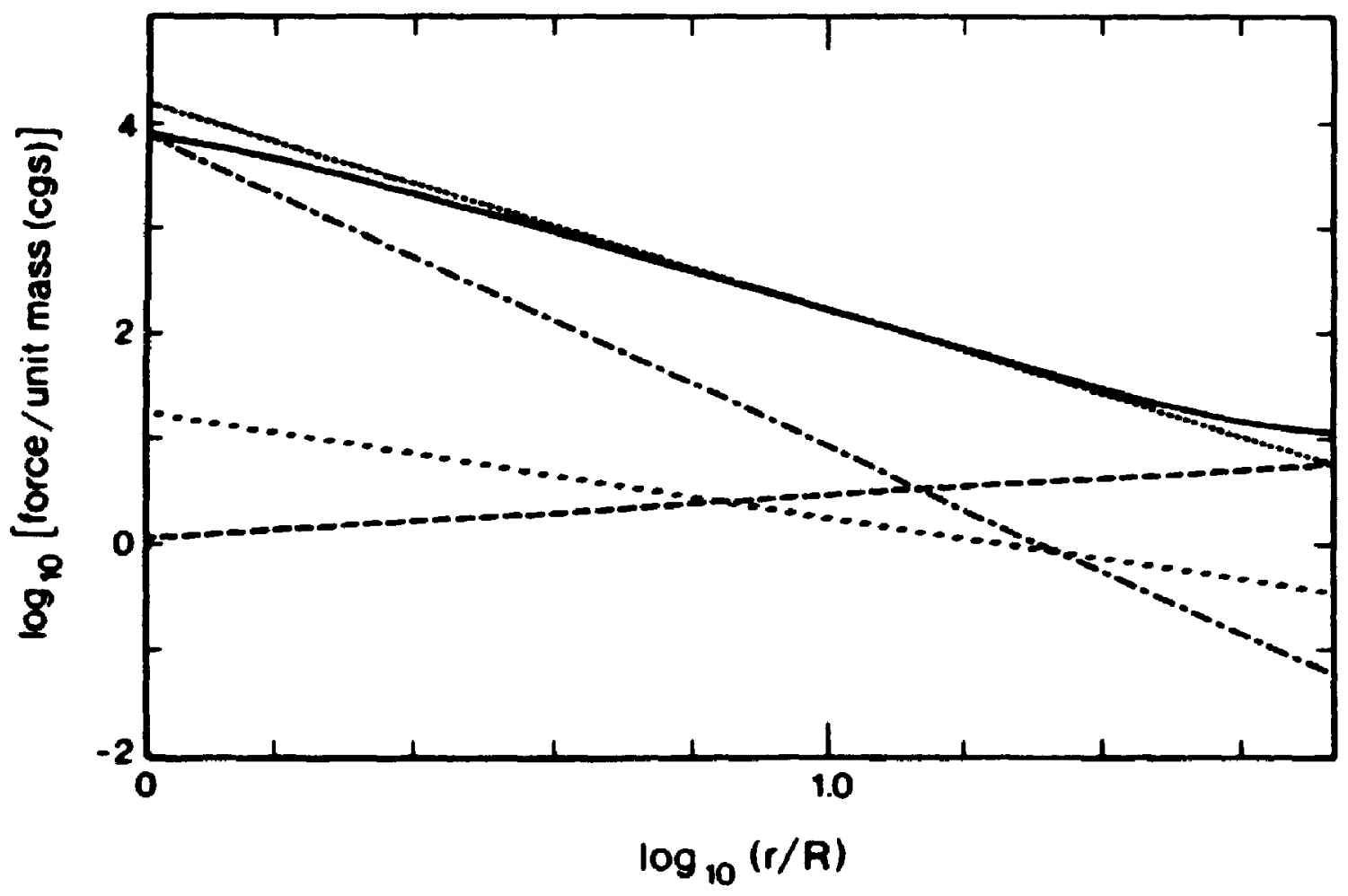

Figure 2.9. The dependence of the force terms on $T / R$ for $\beta$ Mon with $m-$ 0.7. See the captions of Figures 2.2 and 2.8 for additional detalls. 


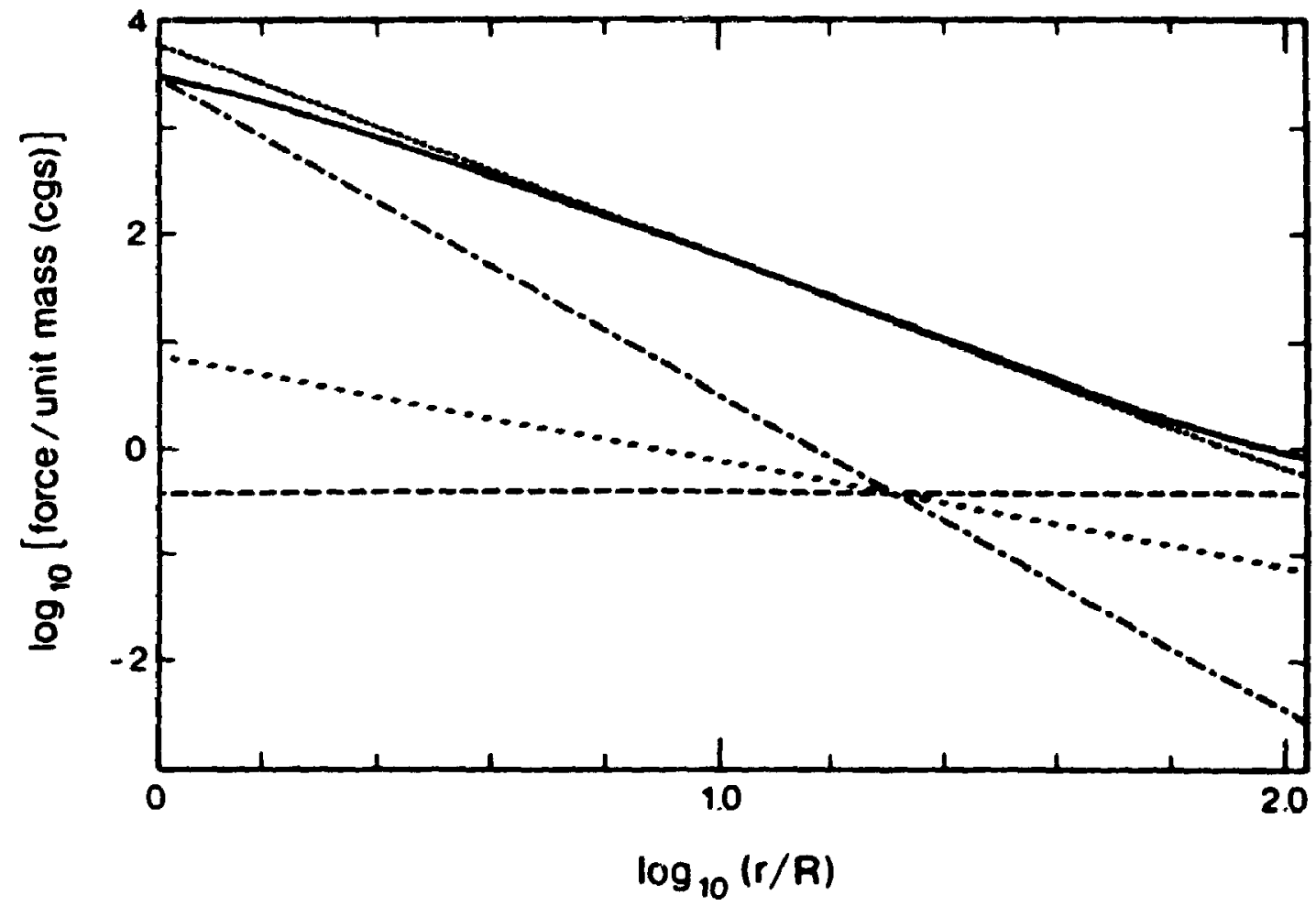

Figure 2-10. The dependence of the force teras on $r / R$ for $E$ Wac with a - 0.5. See the captions of figures 2.2 and $2-8$ for additional detalls. 


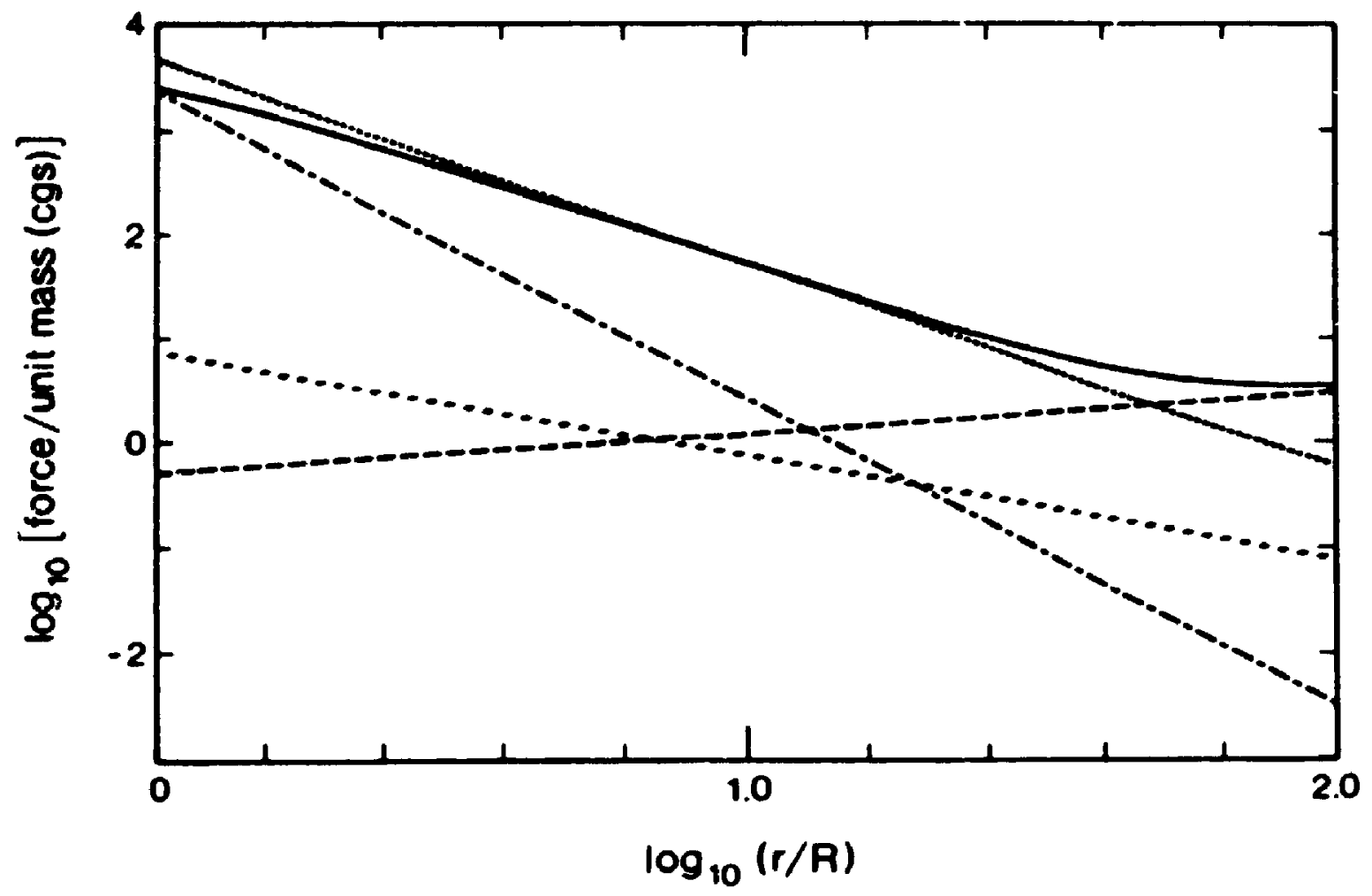

Figure 2-11. The dependence of the force terms on $r / R$ for $\$$ Per with $m$ 0.7. See the captions of Figures 2.2 and 2.8 for additional results. 


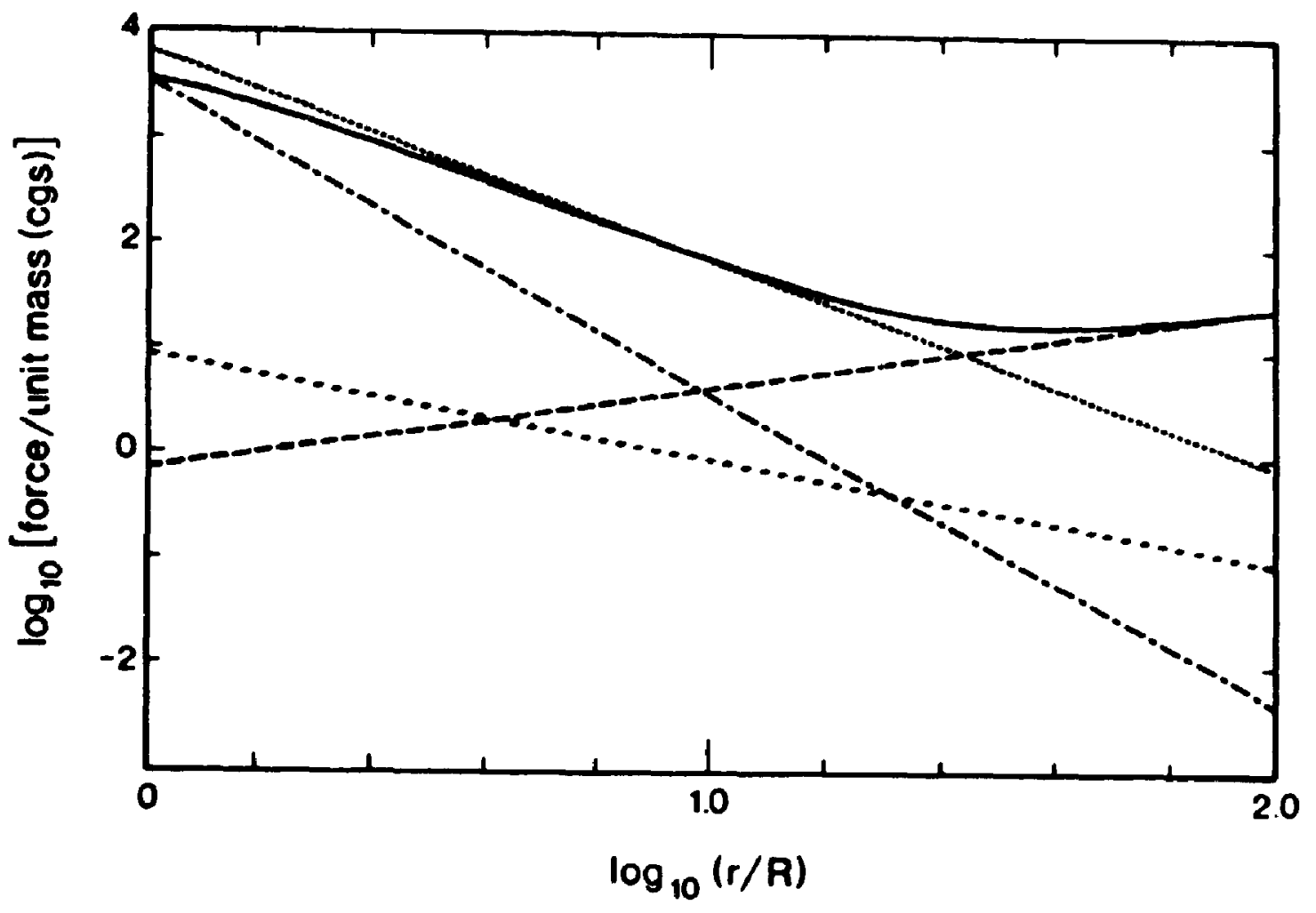

Figure 2-12. The dependence of the force teres on $r / R$ for $\eta$ Tau with a 0.9. See the captions of Figures $2-2$ and 2.8 for additional detasls. 


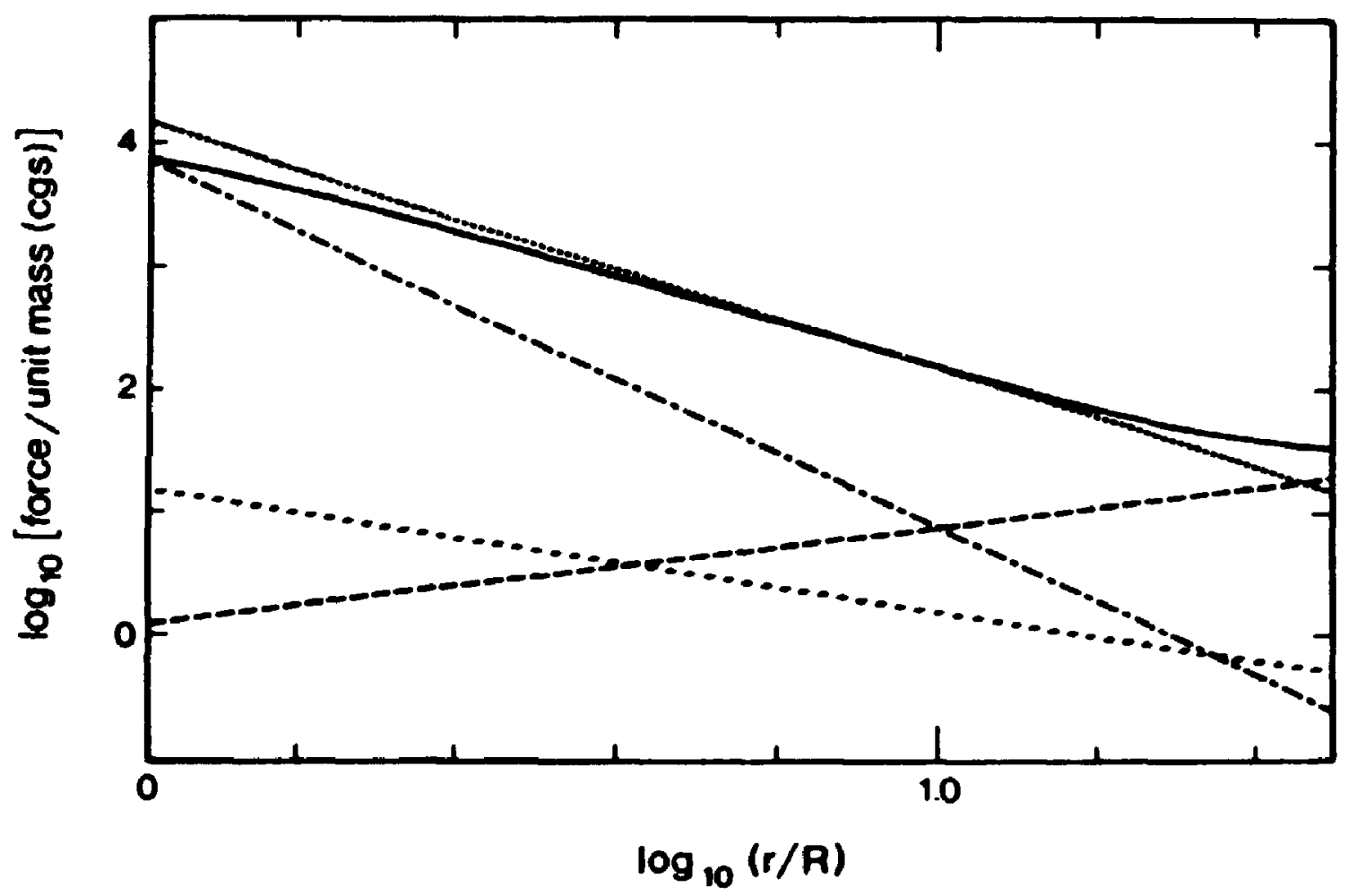

Figure 2-13. The dependence of the force terms on $r / R$ for $\beta$ CHI with 0.9. See the captlons of Figures 2.2 and 2.8 for additional detalls. 
surface of the star if Be stars actually rotate at sone significant fraction of the critical speed. At larger distances from the surface the importance of the centrifugal term depends on the form of $v_{0}(r)$. If angular momentum is conserved the centrifugal term becomes less and less important with increasing distance. On the other hand if $v_{p}(r)$ is Keplerian, the ratio of centrifugal force to gravity is Independent of $r$. Both the beta velocity law with large $\beta$ values and the power velocity law with small w values have the potential to generate velocities of magnitude similar to those deduced from Be star data. For both distributions, VdV/dr is very small near the surface of the star although it does increase outward. Beyond about ten stellar radil, VdV/dr is the major contributor to $F_{x}$. The unknown force, $F_{x}(r)$, clearly depends on the radial velocity distribution, $V(r)$, azimuthal velocity distribution, $V_{\phi}(r)$, and temperature distribution, $T(r)$. However, for different choices of $V(r), V_{\phi}(r)$, and $T(r)$ which are consistent with Be star data, $F_{z}(r)$ has the same qualitative behaviour. Between the surface of the star and a turning point located between 8 and 100 R, F decreases slightly less rapidly than $F_{g}$ so that $F_{x}>F_{q}$ at the turning point. This dependence of $F_{x}$ on $r$ can be approximated by

$$
F_{x}(r)=\frac{C}{r^{2-\varepsilon}}
$$

where $C$ is a constant and $\epsilon$ is a small number. Beyond the turning point $F_{x}$ increases with $r$ rapidly. In some cases this increase continues; in others $F_{x}$ reaches a maximum and then decreases again. The initial rapid increase of $F_{x}$ beyond the turning point makes it dominate all other forces rapidly.

What physical process or processes is/are responsible for $F_{x}$ This 
is the major question to be answered. Since the dependence of $F_{x}$ on distance has been derived, I am certainly in a better position to answer this question. The unknown force $F_{x}(r)$ has a different form between the surface of the star and the turning point compared to its form beyond the turning point. I suggest that two different mechanisms must be considered, one of which dominates interior to the turning point and a different one which dominates the dynamics beyond. Possible candidates for $F_{x}(r)$ are: a) the radiation force from strong lines, b) the radiation force from weak lines, c) a magnetic field, d) wave dissipation, arte) angular momentum transfer by $x$ ans $-f$ a magnetic field or viscous effects; there may well be other candidates. In the following few paragraphs, I shall discuss briefly these possible candidates.

Many years ago Limber \&arlborough (1968) considered the sipport of the envelopes of Be stars and concluded that viscous transfer of angular momentum by a magnetic field is the most likely process by which such support is maintained. Given the fact that Be stars probably do not rotate at the critical speed, it is reasonable to expect that this process may be an important contributor to the dynamics only in the region Interior to the turning point. The anomalously broad $\mathrm{Ca}$ II $\mathrm{K}$ lines in the spectra of some shell stars (Gulliver 1981) inay be evidence that such angular momentum transfer processes operate in the envelopes of Be stars. It seems unlikely that the radiation force due to strong lines operates in the Inner part of Be star envelopes. The dependence of this force on distance from the star is given In Figure 9 of Poe \& Friend (1986). While the magnitude of the force as a function of distance will certainly crange from one star to another, the shape of the force is expected co be qualitatively the same. This shape, specifically a rapid increase to maximun just above the stellar surface and then a decrease similar to the gravitational firce, is decidedly unlike the form of $F_{x}(r)$ 
Interior to the turning point. It does, however, have some resemblance to $F_{x}(r)$ beyond the turning point for the cases in which $F_{x}(r)$ first increases and then decreases with increasing $r$.

The radiation force due to weak lines may be a more promising candidate. Suppose that the total number of weak lines is $\mathbf{N}$ and the mean opacity of each is $x$. The force due to these lines can be expressed as

$$
F_{1}-C \frac{K N}{r^{2}}
$$

where $C$ is a constant which is proportional to the luminosity of the star. This force has the same $r$ dependence as gravity. I assumed

$$
\boldsymbol{N}=\boldsymbol{I} \bullet
$$

where $\epsilon$ is a small number the same as used in equation (2-11), 1.e, the number of weak lines increases only slowly with $r$. Then the foras if $F_{x}$ becomes

$$
F_{x}(r)-C \frac{\kappa N_{0}}{r^{2-e}}
$$

It has the desired dependence on $r$ in the region interior te the turning point.

Wave dissipation has been proposed as a mechanism for generating stellar winds. Hartmann \& MacGregor (1980) discussed the generation of winds in cool stars by Alfven wave dissipation, while Pifpers \& Hearn (1989) suggested that winds in hot stars may also be driven by acoustic waves. In all these cases the source of the waves is some photospheric or subphotospheric phenomenon, e.g., a magnetic field or stellar pulsation. Thus it seems reasonable to expect that wave dissipation may contribute to $F_{x}(r)$ Interior to the turning point, rather than beyond, al though one must first consider the spectrum of waves generated and the damping lengths before ruling out a wave contribution to $F_{z}$ beyond the turning poir:s. 


\section{CHAPTER 3 A POTENTIAL SIMILARITY AND DIFFERENCE BETWEEN Be STARS AND SHELL STARS}

\subsection{INTRODUCTION}

In Chapter 2, I applied my calculation of the unknown force to six well studied Be stars. I noted that among these six Be stars, three were classified by Slettebak (1982) as shell stars and three others as normal Be stars. The spectra of shell stars are systematically different from -hose of Be sicars. Shell stars or Be stars in a shell phase have deep and narrow absorption components in their spectra which nurmal Be stars do not have. This difference suggests that there are differences between the envelopes of Be stars and those of shell stars, either geometrically or physically, or both. However people still do not know what the differences are and what causes these differences. Some consider shell stars to have a more extensive envelope than Be stars (Hutchings 1976). The UV spectra of shell stars and Be stars in shell phases show both a large number of absorption lines and direct evidence for significantly enhanced continuum and line opacity (Slettebak and Carpenter 1983, Beeckmans 1976). Even at optical wavelengths it is sometimes difficult to classify the photospheric spectrum of a shell star due to the large number of metallic absorption lines in the spectrum (Slettebak and Carpenter, 1983). All this evidence suggests that shell stars have more extensive and perhaps denser envelopes than Be stars do. However, Slettebak, Collins and Truax (1991) suggest that shell stars have envelopes which are more compact and have lower electron densities than the envelopes of Be stas based on their recent spectroscopic observations for 41 bright Be stars, among which seven show shell star characteristics. 
The purpose of this chapter is to compare the similarities and differences in $F_{x}(r)$ of these three Be stars and three shell stars so that I may learn more about the dynamical characteristics of the envelopes of these stars.

$3.2 F_{x}$ FOR THREE Be STARS AND THREE SHELL STARS

The parameters of the six stars have already been given in Table 21 The error in determining the indices $m_{0}$ is $\Delta m- \pm 0.1$ (10) for all the stars (Waters 1991).

I have already derived $F_{x}$ for these six stars in Chapter 2 . The force for the six stars are summarized in Figure 3-1. The form of $F_{x}$ of the six stars has similarities as well as differences. Since these six stars have different masses and radii, some of the differences are introduced by the different gravity of the stars. If $F_{x}(r)$ is normalized in terms of gravity for each star, the effect introduced by the different gravitational force will be eliminated. In this case, equation (2-1) becomes

$$
F_{x g}-\frac{F_{x}}{F_{g}}-\frac{V}{F_{g}} \frac{d V}{d r}-1-\frac{F_{\mathrm{p}}}{F_{g}}-\frac{F_{\mathrm{c}}}{F_{\mathrm{g}}}
$$

The unknown force normalized, $F_{x g}$, for the six stars are illustrated in Figure 3-2. The uncertainty in determining $m_{0}$ will introduce some uncertainty in $F_{x}$, and therefore in $F_{x g}$. In order to see how $F_{x g}$ is influenced by the exrors in determining the velocity index $m_{0}$, I have used two vertical bars for each curve in Figure 3-2. The upper end of the error bar corresponds to the normalized $F_{x}$ with $m=m_{0}+0.1$; and the lower end, the normalized $F_{x}$ with $m=m_{0}-0.1$. Since $F_{x}$ depends on both the index $m$ and the distance $r$, the length of the bar varies. The separation 


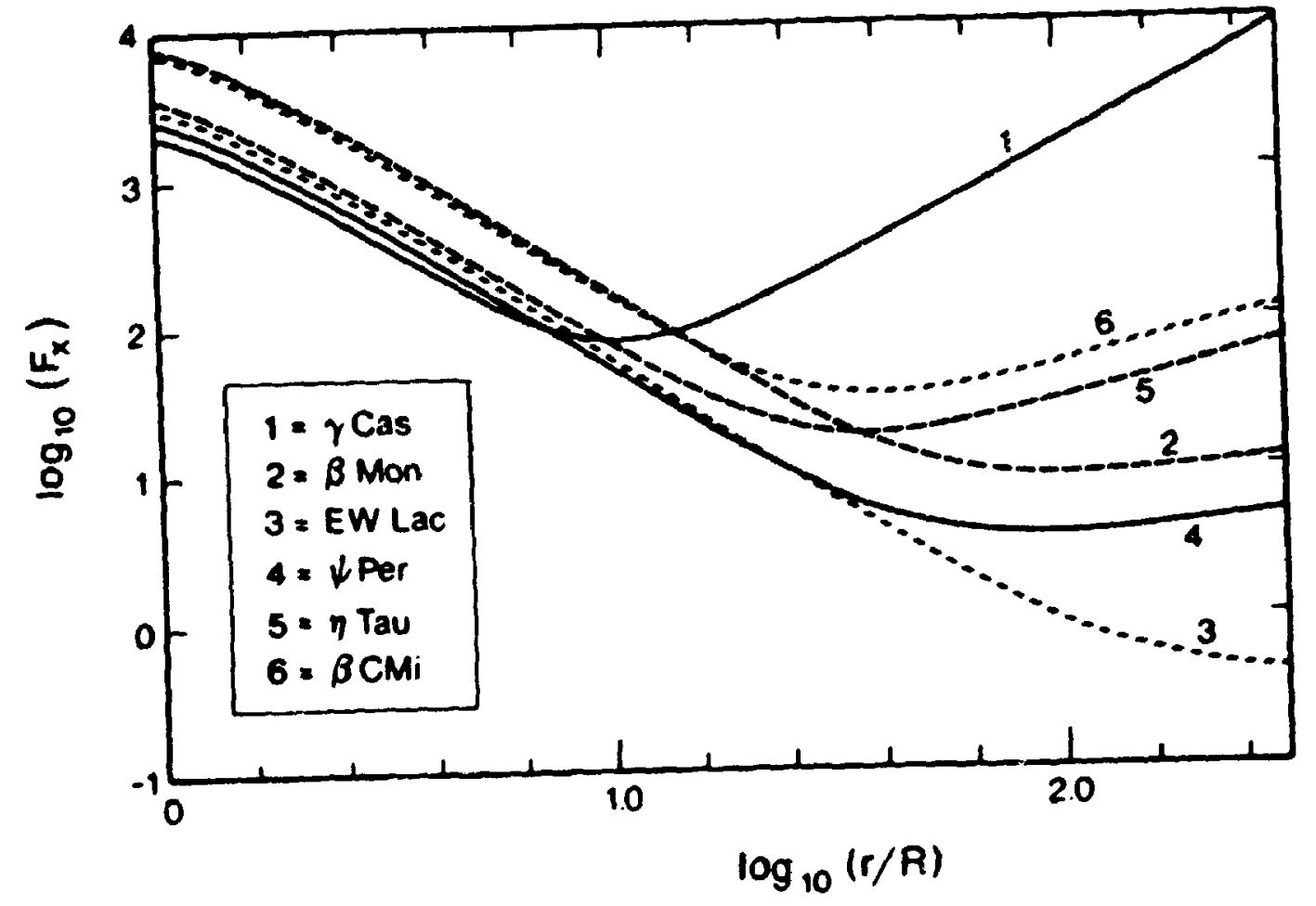

Figure 3-1. $F_{2}(r)$ for the six stars. 


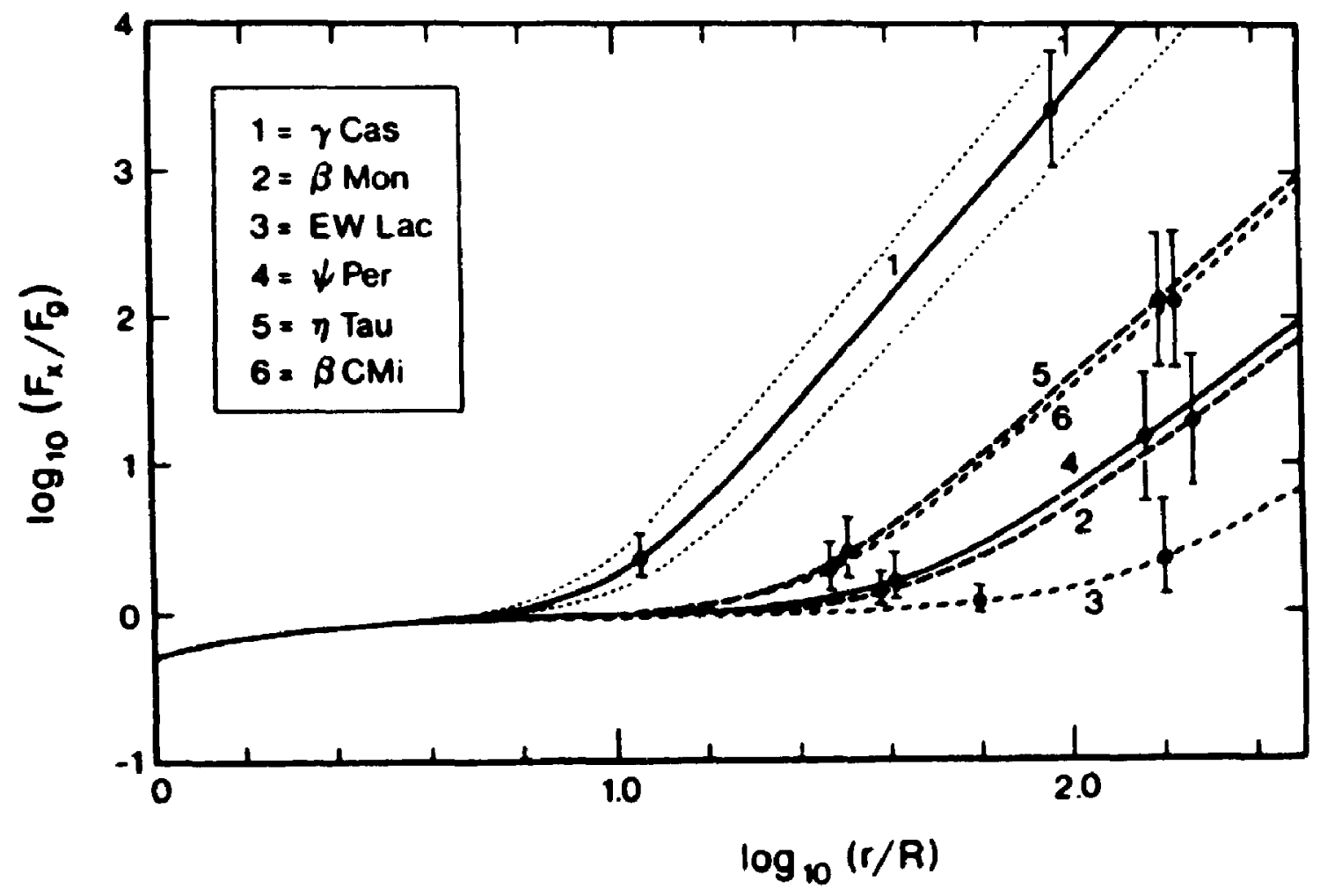

Figure 3-2. $F_{x}(r)$, normalized in terms of gravity, for the six stars. The Be stars are: $\gamma$ Cas, $\eta$ Tau and $\beta$ CMI; the shell stars are: $\beta$ Mon $A$, EW Lac and $\phi$ Per. For $y$ Cas I show the region bounded by two dotted lines in which $F_{x}(r)$ can occur due to the uncertainty. An, in $a_{0}$ : the upper corresponds to $m_{0}+\Delta m$, and the lower corresponds to $n_{0}-\Delta m$. To avoid crowding I use only two vertical bars to represent the extent of this region for the other 5 stars. 
between $F_{x g}(r)$ corresponding to $m-m_{0}+0.1$ and that corresponding to $m$ - $m_{0}-0.1$ decreases as $r$ decreases, and approaches zero as $r$ approaches the turning point.

The normalized $F_{x}$ is essentially the same for all six stars between the star and a particular points which I called the turning point in Chapter 2. They all appear to be flat with only a slow increase with increase in $r$. Beyond the turning point, $F_{z g}$ starts to increase rapidly. From Figure 3-2, the Be star $\gamma$ Cas is the first one to reach the turning point and the shell star EW Lac is the last one to reach it. The normalized $F_{x}$ for the other four stars lies between these two stars, with $F_{x g}$ for the two Be stars, $\eta$ Tau and $\beta$ CAi reaching the turning point earlier than the two she11 stars, $\beta$ Mon $A$ and $\psi$ Per, although the difference is not as significant as the difference between $F_{x g}$ for $\gamma$ Cas and EW Lac.

\section{3 DISCUSSION}

The fact that the normalized $F_{x}$ interior to the turning point is similar for Be stars and shell stars suggests that the mechanism that drives winds in this region is same for both Be and shell stars. It is not clearly known what this mechanism is. I suggested several candidates for it in Chapter 2. Two of them are the presence of a weak magnetic field and the radiation pressure due to weak lines. In Chapter 4, I shall consider how a weak magnetic field ( $B_{0}-10$ gauss) can influence the dynamics in the equatorial plane of the envelopes of Be stars; and in Chapter S, I shall consider how a radiation force due to optically thin lines can drive the envelopes in the equatorial plane. Each of these mechanisms is a potential candidate for the driving mechanism inside the 
turning point. Whataver the mechanism is, it seens to be the same for both Be stars and shell stars.

In Chapter 2, I suggested that a second driving mechanism takes over the first one at the turning point. I do not yet know what this second mechanism is. From both Figures $3-1$ and $3-2$ I note that this force increases very fast and becomes the dominant force. The large $F_{x}$ corresponds to a large acceleration of the wind, therefore producing a rapid drop in density. If I were to define this distance at which the density drops dramatically as the radius of the envelope, the turning point would then be the radius of the effective emitting envelope. The fact that $F_{x}$ for iie Be star $\gamma$ Cas reaches turning point at much smaller distance than that for the shell star EW Lac probably implies that $\boldsymbol{\gamma}$ Cas has a smaller effective emitting envelope than does EW Lac. If the three Be stars are considered as a group and three shell stars as the other, the same conclusion can be drawn.

The turning point for $y$ Cas is only about eight stellar radil from the surface of the star and $F_{x}$ is much higher for $\gamma$ Cas than for other 5 stars. Since $\gamma$ Cas is the hottest star in the sample. It is very likely that the radiation force due to the optically thick lines is responsible for the rapid increase in $F_{\alpha}$ for the star.

The reality of the separation between tine Be and shell stars depends on whether the shell stars were actually in a shell phase when the continuum data were obtained. Recall I am using Slettebak's classification of either $B e$ and/or shell for the six stars, which was based upon the appearance of the spectrum of each in the period 1979-80 (Slettebak 1982). The continum data, however, were obtained at a varlety of times: the IRAS data in 1983, the radio data in 1986 and 1987-88, and 
the millimeter data in August 1989 (see Waters et al, 1991 for details). Fortunately slettebak et al. (1991) have reobserved a number of Be stars, including the six stars considered above, In the vicinity of the first 3 Balmer lines during two perlods January and October, in 1989. The star $\psi$ Per was observed in both January and October. A sharp, central absorption component is visible in each of $\mathrm{Ha}, \mathrm{H} \beta$ and $\mathrm{H \gamma}$, which extends well below the local continuum level in $\mathrm{H} \beta$ and $\mathrm{H} \gamma$. Only small changes in the two spectra were noted by Slettebak et al. The star $\beta$ Mon A was observed only in October. Sharp, central absorption components are present in each Balmer line and those in $\mathrm{H} \beta$ and $\mathrm{H} \gamma$ extend below the local continuum level. EW Lac was observed only in January. Sharp, narrow central absorption components extending below the continum level were present in all 3 Balmer lines. All the Be stars were observed both in January and October. None shows any evidence for shell absorption components in any of the Balmer lines. The data of Taylor et al. (1990) also confirm that the three shell stars did show shell absorption components when the radio observations were obtained. Thus there is evidence that slettebal.'s classification of $\mathrm{Be}$ or shell may still be applicable to the six stars in my sample at the time when some, perhaps all, of the continuum data were obtained.

There are also other factors which influence the location of the turning point, and thus the reality of the separation between the Be and shell stars. The initial velocity is one of these. In my calculation I assumed it to be half of the sound speed at the surface for each star. But its value is poorly known since none of the observations provides direct information about it. I did some experiments in which I let $v_{0}$ vary from 0.1 to 0.9 of the sound speed to see how the choice of $v_{0}$ influenced 
$F_{x g}$. I found that as $V_{0}$ increases the location of the turning point approaches the surface of the star and therefore $F_{x g}$ will be higher. When $V_{0}$ decreases the reverse occurs. When I increased $V_{0}$ for shell stars from 0.5 to 0.9 of the sound speed and at the same time reduced $v_{0}$ for Be stars from 0.5 to 0.1 of the sound speed, the separation between Be and shell stars was greatly reduced. However there is some evidence indicating that the opposite situation is more realistic. The line profile calculations by Marlborough and Cowley (1974) and Poeckert and Marlborough (1978) indicated that the initial velocity has to be very low (the order of 0.1 sound speed) to produce the profiles of shell stars: otherwise the profiles will look like type I P Cyg profiles. This doesn't necessarily mean that the initial velocity of Be stars is higher than that of shell stars. However it does provide some evidence that the opposite situation is unlikely. 
CHAPTER 4 THE INFLUENCE OF A WEAK MAGNETIC FIELD

ON THE AZIMUTHAL MOTION OF A SLOWLY EXPANDING WIND

\subsection{INTRODUCTION}

In Chapter 2, several mechanisms were suggested which could possibly explain the driving of Be star envelopes. One of them is the presence of a weak magnetic fleld.

A magnetic field can play an important role in transferring angular momentum from a rotating star to an extended, expanding envelope. Weber and Davis (1967) constructed a magnetic wind model for the solar wind, and Mestel (1968) showed how centrifugal forces, controlled by a magnetic field, can drive a wind. Limber and Marlborough (1968) considered what roles a magnetic field might have in Be star envelopes and concluded that transfer of angular momentum from the magnetic field to the matter is the most likely process by which support of the envelope is maintained.

A detailed model for Be stars in which both a magnetic field and a radiation force due to optically thick lines are included was first constructed by Poe \& Friend (1986). In their study, they assumed that the radial component of the magnetic field at the surface varied from 25 gauss to 400 gauss. The radial velocity struccure of the wind in their model is basically the same as that of the radiation driven wind model (Friend \& Abbott 1986) in which only thick lines are considered, i.e., the velocity increases so rapidly that a speed of order $1000 \mathrm{~km} \mathrm{~s}^{-1}$ is attained within 1-2 stellar radif. As I discussed in Chapter 2, a velocity structure like this will not be able to explain Ha profiles, Infrared excess etc. In Poe and Friend's model, the azimuthal velocity distribution $v_{\phi}$ is influenced by the presence of the magnetic field. Because of the transfer of angular 


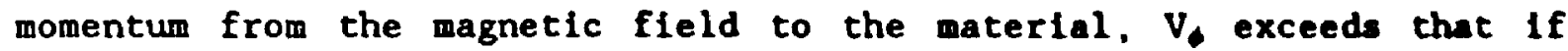
angular momentum is conserved. For a model with $B_{0}-50$ gauss, $V_{0}$ is about twice as high as that if angular momentum is conserved.

The search for the magnetic fields in Be stars was summarized by Barker (1987). Up to now, all observations that have been carried out give null results for both the longitudinal and azimuthal components, with upper limits between 75 to 300 gauss. These results seem to suggest an upper limit for the strength of the magnetic field if one is going to construct a Be star model including a magnetic field.

As I discussed in Chapter 2, the envelopes of Be stars are slowly expanding in the equatorial plane. At the same time, one should renember that the magnetic field, if it does exist, is not a strong one. It is interesting to see if a weak magnetic field will influence a slowly expanding envelope. If yes, what is the influence? In this chapter, I shall investigate how a weak magnetic field ( $B_{0}-10$ gauss) influences a wind, especially a Be star wind, which is assumed to be slowly expanding in the equatorial plane.

The remainder of this chapter is organized as follows. In $\$ 4.2$, I consider the dynamics of Be star envelopes in the equatorial plane when a magnetic field is present. In $\$ 4.3$ I calculate the distribution of the azimuthal velocity and the azimuthal magnetic field for models with various mass loss rates, radial velocity distributions and strengths of the radial component of magnetic field at the surface of the star. And in $\$ 4.4$, I discuss what kind of $F_{x}$ is needed to produce the assuned radial velocity distribution for two models considered in 84.3 . 


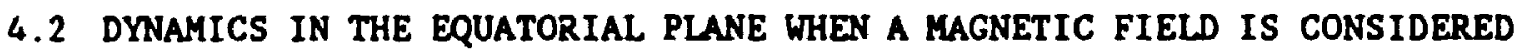

I will use the same system of coordinates as I used in Chapter 2. I also adopt the same assumption about the symmetry of the envelope, 1.e., the envelope is symmetrlc about $z$ axis and the equatorial plane.

Following Weber \& Davis (1967), I assume the meridion 1 components of all variables and the meridional gradient of all variables to be zero in the equatorial plane. The velocity and magnetic field will be

$$
\vec{V}=V_{r}(r)+V_{0}(x) \phi
$$

and

$$
\vec{B}-B_{r}(r) P+B_{\phi}(r) \Phi
$$

Under these assumptions, the radial component and azimuthal component of the equation of motion in the equatorial plane can be written as

$$
V_{x} \frac{d}{d r} V_{z}-F_{\mathrm{q}}+F_{\mathrm{p}}+F_{\mathrm{c}}+F_{\mathrm{ax}}+F_{\mathrm{x}}
$$

and

$$
\frac{V_{x}}{r} \frac{d}{d r}\left(r V_{0}\right)-F_{n}
$$

where $F_{g}, F_{p}, F_{c}$ and $F_{x}$, as in Chapter 2 , are respectively the forces arising from gravity, gas pressure, rotation and some unknown force/forces that could be responsible for driving Be star envelopes; and

$$
F_{m}=-\frac{B_{\phi}}{4 \pi \rho r} \frac{d}{d r}\left(r B_{\phi}\right)
$$


and

$$
F_{m}-\frac{B_{x}}{4 \pi \rho r} \frac{d}{d r}\left(r B_{\phi}\right)
$$

are the radial and azimuthal components of the magnetic force, respectively.

Assuming that the wind is a perfect conductor and in a steady state. I can obtain the following expressions (see Barker Marlborough 1982 for details):

$$
\begin{gathered}
B_{x}(r)-B_{0}\left(\frac{R}{r}\right)^{2}, \\
B_{\phi}(r)-B_{0} \frac{\lambda V_{c}\left(1-\frac{r^{2}}{r_{A}^{2}}\right) R}{r V_{A}\left(1-\frac{r^{2} V_{x}}{r_{A}^{2} V_{\lambda}}(1+f),\right.} \\
V_{\phi}(r)=\frac{\lambda V_{c} r\left(1-\frac{V_{x}}{V_{A}}\right)}{R_{0}\left(1-\frac{V_{x} r^{2}}{V_{A} r_{A}^{2}}\right)}(1+f),
\end{gathered}
$$

where $B_{0}$ is the radial component of magnetic field at the surface of the star; $V_{e}$ is the critical rotational velocity; $\lambda$ is the ratio of the rotational velocity $V_{r o t}$ to the critical rotational velocity; and $r_{\lambda}$ and $V_{\lambda}$ are the Alfven radius and the Alfven velocity, respectively, which are related by the equation 


$$
V_{\lambda} r_{\lambda}^{2}-\frac{B_{0}^{2} R^{2}}{\dot{M}}
$$

Once $B_{0}$. the strength of the magnetic field at the surface, $\dot{M}$, the mass loss rate and radial velocity distribution are given, $r_{\lambda}$ can be deterrined using equation (4-10) using the assumed form for $V_{v}$. In equations (4-8) and (4-9) $f$ is the dimensionless parameter introduced by Barker \& Marlborough (1982) for the situation when the radial velocity at the reference level is not zero. In this work it is chosen as the surface of the star. Barker Marlborough show that

$$
f=\frac{V_{x, 0^{1 B}} V_{t, 0}}{V_{t, 0_{0}}}
$$

where $v_{z, 0}$ and $v_{\phi, 0}$ are, respectively, the radial and azimuthal components of the velocity at the surface; and $B_{\diamond .0}$ is the azimuthal component of the magnetic field there.

I introduce a dimensionless distance

$$
x-\frac{r}{R}
$$

and velocity

$$
y=\frac{v}{a}
$$

where $R$ is the radius of the star and a is the isothermal sound speed of the wind. Equations $(4-7),(4-8)$ and (4-9) can be rewritten as

$$
B_{z}(x)-B_{0} \frac{1}{x^{2}}
$$




$$
B_{\phi}(x)=-B_{0} \frac{\lambda y_{\text {exi }}\left(1-\frac{x^{2}}{x_{A}^{2}}\right)}{x y_{\lambda}\left(1-\frac{x^{2} y}{x_{A}^{2} y_{\lambda}}\right)}(1+f)
$$

and

$$
y_{\phi}=\frac{\lambda y_{\mathrm{cr} 1} x\left(1-\frac{y}{y_{\lambda}}\right)}{1-\frac{x^{2} y}{x_{\lambda}^{2} y_{\lambda}}}(1+f)
$$

Once the radial velocity distribution, the mass loss rate and the magnetic field at the surface are given, I can then cetermine $B_{\phi}:$ ad $y_{\phi}$ from equations $(4-10),(4-15)$ and $(4-16)$.

4.3 Distribution of the Azimuthal Velocity and the Azimuthal Magnetic Field

I shall use the $\beta$ law velocity distribution (Equ. 2-9) and the power law velocity distribution (Equ. 2-10) for my study in this chapter. Although neither of them may be correct, they can describe the characteristics of the slow expansion of Be star envelopes.

In a1l of the following calculations, the stellar par. ters used are assumed to be similar to a star like $\gamma$ Cas: $M-16 M_{0}, R-10 R_{0}, L=$ $5.4 \times 10^{\circ} \mathrm{L}_{0}, \mathrm{~T}_{\text {eff }}-25,000^{\circ} \mathrm{K}$. I also assume the rotational velocity at the equator of the star to be 0.7 of the critical velocity and the wind temperature, $0.6 \mathrm{~T}_{\text {off }}$ - 


\subsubsection{The Beta Law}

In equation (2-9) for the beta velocity law, I assume that $v_{0}-1500$ $\mathrm{km} \mathrm{s} \mathrm{s}^{-1}$ and $V_{c}-0.5$ times he sound speed of the wind. The parameters of the four models are given ir. Table 4-1. Column 1 gives the model numbers; column 2 gives the $\beta$ value; column 3 gives the mass loss rate; column 4 , the radial component of the magnetic field at the surface; column 5 , the Alfven radius; and column 6 , the total angular momentum per unit mass (in units of the sound speed times stellar radina). Three models have large $\beta$ values (models 1,3 and 4 with $\beta-15$ ). For comparison, I also consider a model with small $\beta$ (model 2 with $\beta-1$ ). A $\beta$-law velocity distribution with $\beta-1$ is a typical velocity distribution for a radiation driven wind model arising from scattering in optically thick lines (Friend \& Abbott, 1986).

In Figure 4-1 I show the distribution of azimuthal velor-ty for the models in Table 4-1. The azimuthal velocity distribution for angular momentum conservation is also given (curve 5). The straight line in Figure 4-1 is the azimuthal velocity distribution of solid body rotation. Surprisingly, the azimuthal velocities of models 1,3 and 4 increase faster than that for solid body rotation. There is little difference between the azimuthal velocity distribution of model $2(\beta-1)$ and that for angular momentum conservation (model 5 ). This neas that for a wind with a rapidly increasing radial veloc:ty, the presence of a weak magnetic field does not influence the azimuthal motion of the wind significantly. On the contrary, for a wind with a large $\beta$, i.e., a slowly expanding wind, even a weak magnetic fleld can influence the azimuthal velocity dramaticaliy. For models with $\beta-15$, the azimuthal velocity can reach as high as 4 times (model 1), 24 times (model 3) and 12 times (model 4) the 
TABLE 4-1

MOOE Paraheters - $\beta$-Lans

\begin{tabular}{|c|c|c|c|c|c|}
\hline & $\beta$ & $\dot{\boldsymbol{M}}$ & $B_{0}$ (gauss) & $\mathbf{r} \sqrt{ } \mathbf{R}$ & Ltotel \\
\hline & & $\left(10^{-8} \mathrm{Mo} /\right.$ year $)$ & & & $(c . g \cdot s / 8)$ \\
\hline model 1 & 15 & 1.0 & 5.0 & 3.46 & 556 \\
\hline model 2 & 1 & 1.0 & 5.0 & 1.11 & 33 \\
\hline model 3 & 15 & 5.0 & 5.0 & 2.28 & 3197 \\
\hline $\begin{array}{l}\text { model } 4 \\
\text { model } 5 *\end{array}$ & 15 & 1.0 & 2.5 & 2.49 & 1657 \\
\hline
\end{tabular}

* In this model the azimuthal velocity distrlibution is calculated assuming angular momentum is conserved. Therefore it is Independent of $\dot{H}, B_{0}$ and $v_{\mathbf{x}}$. 
rotational velocity.at the surface of the star, respectively. The curve indicated by crosses is the local escape speed. It can be seen that the azimuthal velocity for models with large $\beta$ (models 1,3 and 4 ) exceeds the local escape speed very soon while that for the model with a small $\beta$ (model 2) is always below the local escape speed.

The distribution of tho azimuthal component of the magnetic field is given in Figure 4-2. For a wind with a rapidly increasing radial velocity (model 2), the azimuthal component of the magnetic field is very small. But for a slowly expanding wind (models 1, 3 and 4), a strong azimuthal component of the magnetic field is induced. Compared with the radial component of the magnetic field, the azimuthal component is so large that the magnetic field is essentially a toroidal one.

In Figure 4-3, I illustrate the transfer process of the angular momentum from the magnetic field to the material. At the surface of the star most argular momentum is in the material for a wind with a rapidy increasing radial velocity; while for a slowly expanding wind most angular momentum is in the magnetic field. With increasing distance, the angular momentum is transforred from the magnetic field to the material for both the rapidly expanding and slowly expanding winds. For the former, the angular momentum in the material does not increase much since most angular momentum is already in the material. For the later, however, the material gains a significant amount of angular momentum in the first a few stellar radii. At about three stellar radii, the material has the same amount of angular momentum as does the magnetic field. With continued transfer, over ninety percent of the total angular momentum is in the material at ten stellar radil.

To understand what makes the azimuthal velocity of a $\beta$-law velocity 


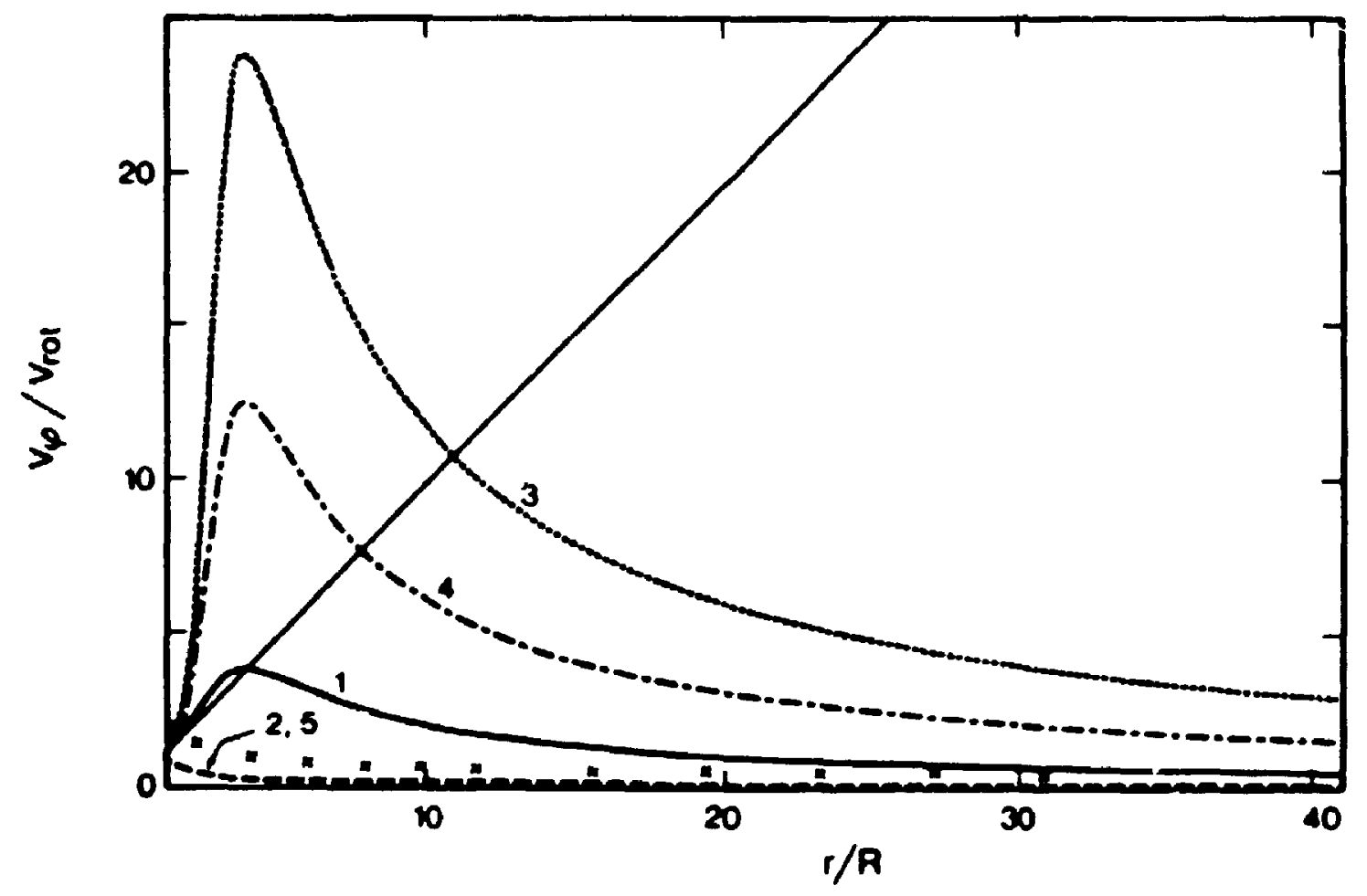

Figure 4-1. The distribution of azimehal velocity for four $\beta$-lav velocity distributions given in Table 4-1. The numbers above the curves Indicate the corresponding nodel numbers in Table 4-1. The azlnuthal velocity distribution for angular montus conservation is given by curve 5 . The stralght line is the aziauthal velocity distribution for solid body rotetion. The curve indiceted by crosses is the ratio of the local escape speed to the stellar rocation speed. 


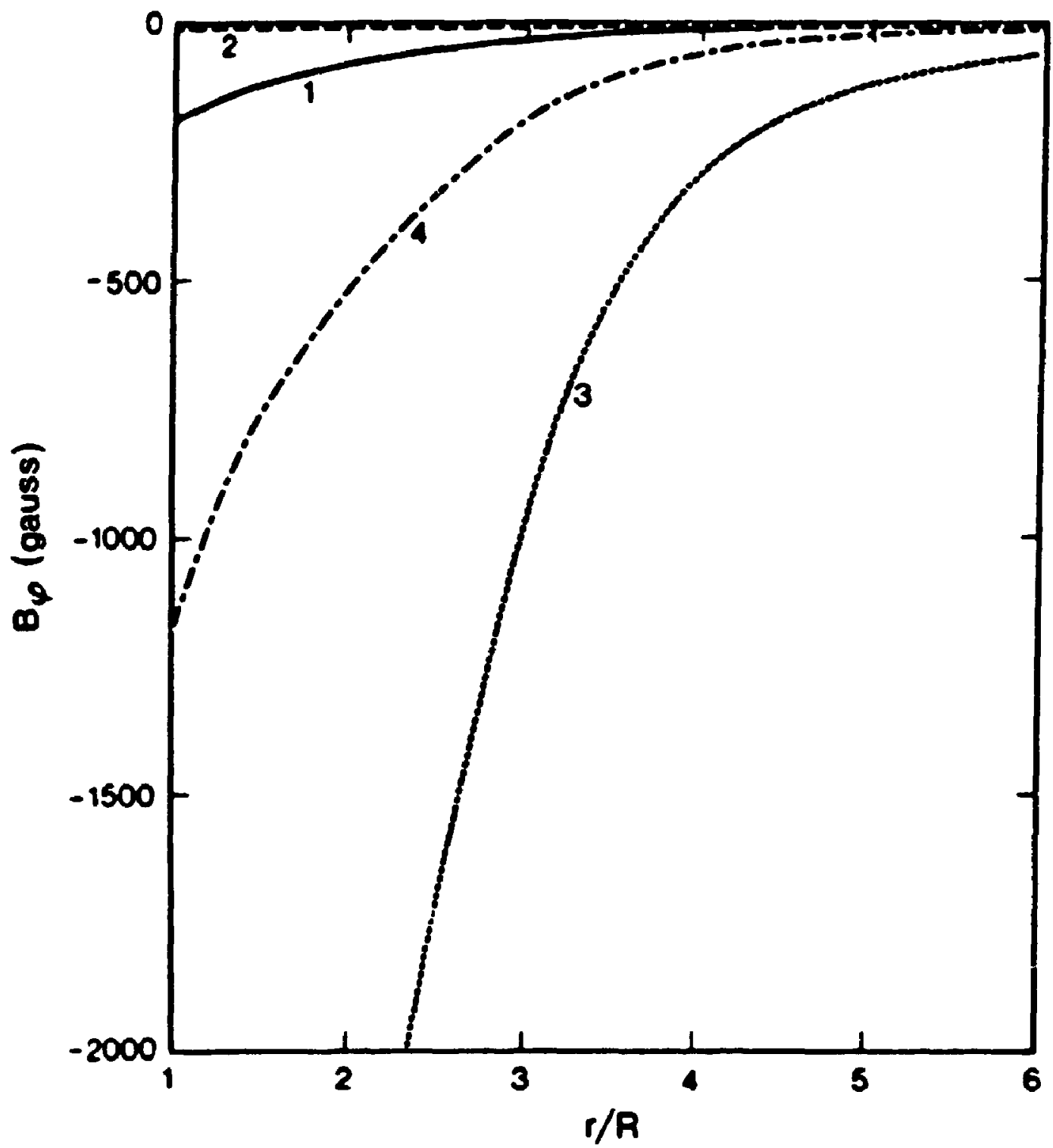

Figure 4-2. The distribution of the azimuthal component of the magnetic field for four $\beta$-law velocity distributions given in Table 4-1. The numbers above the curves indicate the corresponding model numbers in Table 4-1. 


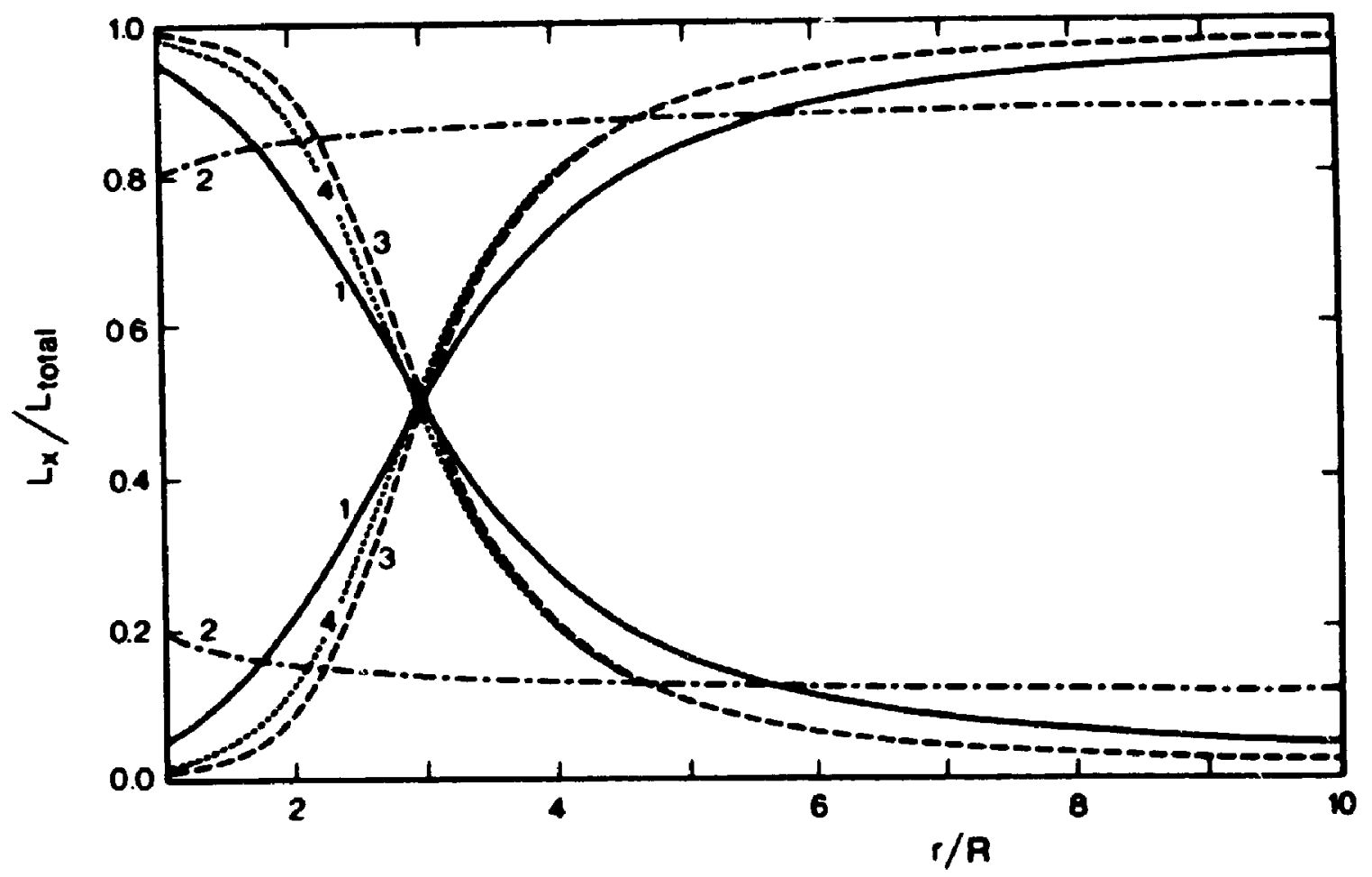

Figure 4-3. The transfer process of angular momentun frod the magnetic fleld to the material for four models given in Table 4-1. The numbers indicate the corresponding nodel numbers in Table $4 \cdot 1$. The increasing curves give the percentage of the angular monentun in the matter over the total angular momentum; and the decreasing curves give that of the angular momentu. in the magnetic fleld over the rotal angular monentun. 
so large, I investigate the value of $f$ in equations (4-8) and (4-9). Substituting equations $(4-8)$ and $(4-9)$ to the definition of $f$, equation (4-11) and using the dimensionless varlables, I have

$$
f=\frac{y_{0}\left(1-\frac{1}{x_{\lambda}^{2}}\right)}{y_{A}\left(1-\frac{y_{0}}{y_{A}}\right)}
$$

where $y_{0}$ and $y_{A}$ are, respectively, the dimensionless radial velocity at the surface and Alfven point. For model 3 , with $\beta=15, B_{0}-5$ gauss and $\dot{M}=$ $5 \times 10^{-8} M_{0} y^{-1}$. I have $x_{n}=2.28$, and surprisingly

$$
\begin{gathered}
y_{\lambda}-y_{0}+\left(y_{0}-y_{0}\right)\left(1-\frac{1}{x_{\lambda}}\right)^{\beta} \\
=0.518
\end{gathered}
$$

i.e.

$$
\frac{y_{\lambda}}{y_{0}}-1.036
$$

Equation (4-21) tells us that over 1.28 stellar radii (from the surface to the Alfven point) the radial velocity increases only 3.68 from the its surface value. This may be one of the unrealistic points of the $\beta$-law velocity. Substituting the value of $y_{\lambda}$ into equation (4-19), I obtain a value of $f$ as large as 22 . This large value of $f$ leads to both a large azimuthal velocity and a large azimuthal component of magnetic field (see equations $4-15$ and $4-16)$.

\subsubsection{The Power Law Velocity}

The power law velocity is given in equation (2-10). I assume $v_{0}$ to 
TABLE 4-2

Moon Parureters - Pongr Lans

\begin{tabular}{|c|c|c|c|c|c|c|}
\hline & $\mathbf{m}$ & & $\dot{\boldsymbol{M}}$ & $B_{0}$ (gauss) & $r_{\mathbb{N}} \mathbf{R}_{\mathbf{.}}$ & Ltotel \\
\hline & & $\left(10^{-8}\right.$ & H /year) & & & \\
\hline model 6 & 0.7 & & 1.0 & 1.0 & 1.03 & 107 \\
\hline model 7 & 0.7 & & 1.0 & 2.0 & 1.72 & 194 \\
\hline model 8 & 0.7 & & 1.0 & 5.0 & 3.39 & 517 \\
\hline model 9 & 0.7 & & 1.0 & 10.0 & 5.67 & 1216 \\
\hline model 10 & 0.7 & & 1.0 & 20.0 & 9.47 & 3033 \\
\hline
\end{tabular}

TABLE $4-3$

Mooel Parafeters - Pondr laus

\begin{tabular}{|c|c|c|c|c|c|}
\hline & $\mathbf{m}$ & $\dot{\boldsymbol{M}}$ & $B_{0}$ (gauss) & $\mathbf{r} \boldsymbol{R}$ & Leotal \\
\hline & & Mo /year) & & & \\
\hline model 11 & 0.7 & 0.1 & 5.0 & 7.96 & 2213 \\
\hline model 12 & 0.7 & 0.5 & 5.0 & 4.38 & 786 \\
\hline model 13 & 0.7 & 1.0 & 5.0 & 3.39 & 518 \\
\hline model 14 & 0.7 & 2.0 & 5.0 & 2.62 & 349 \\
\hline model 15 & 0.7 & 5.0 & 5.0 & 1.87 & 216 \\
\hline model 16 & 0.7 & 20.0 & 5.0 & 1.12 & 116 \\
\hline
\end{tabular}


TABLE 4.4

Model Parneters - Pondr Las

\begin{tabular}{|c|c|c|c|c|c|c|}
\hline & $\mathbf{a}$ & & $\dot{\mu}$ & $B_{0}$ (gauss) & $r_{\boldsymbol{N}} \mathbf{R}_{\boldsymbol{*}}$ & $L_{\text {totel }}$ \\
\hline & & $\left(10^{-8}\right.$ & No /year) & & & \\
\hline model 17 & 0.3 & & 1.0 & 5.0 & 4.19 & 1302 \\
\hline model 18 & 0.5 & & 1.0 & 5.0 & 3.74 & 749 \\
\hline model 19 & 0.7 & & 1.0 & 5.0 & 3.39 & 518 \\
\hline model 20 & 1.0 & & 1.0 & 5.0 & 3.00 & 350 \\
\hline model 21 & 1.2 & & 1.0 & 5.0 & 2.80 & 286 \\
\hline model 22 & 1.5 & & 1.0 & 5.0 & 2.57 & 225 \\
\hline
\end{tabular}


be 0.5 times the sound speed of the wind. The parameters for the d:fferent models are given in Tables $4-2,4-3$ and $4-4$. The structure of Tables 4-2, 4-3 and $4-4$ is basically the same as Table 4-1 except that column 2 now gives velocity indices for the power law Instead of $\beta$ for the $\beta$ velocity law. In order to study how each of the three parameters. $H$, $B_{0}$ and $m$ influences the dynamics systematically, I only change one parameter at one time and keep the other two constant. In Table 4-2, $B_{0}$ varies while $m$ and $\dot{\mu}$ remain the same; in Table 4-3, $\dot{\mu}$ varies while $m$ and $B_{0}$ remain the same; and in Table 4-4, m varies while $\dot{M}$ and $B_{0}$ remain the same. Figures $4-4$ and $4-5,4-6$ and $4-7$, and 4-8 and 4-9 give the azimuthal velocity distribution and the azimuthal component of the magnetic field, respectively, for models in Tables 4-2, 4-3 and 4-4.

Results for the models with fixed m -0.7 and $\dot{M}-10^{-8} \mathrm{M}_{0} \mathrm{y}^{-1}$ and different $B_{0}$ are given in Table $4-2$ and Figures $4-4$ and $4-5$. As $B_{0}$ increases, both the Alfven radius and the total angular momentum per unit mass increase. The distribution of the azimuthal velocity is quite sensitive to the value of $B_{0}$. In general, the larger is $B_{0}$, the higher is $v_{\phi}$. The behavior of $v_{0}$ also depends on $B_{0}$. When $B_{0} \geq 2$ gauss, $v_{\phi}$ increases at first to its maximum at about the Alfven point and then

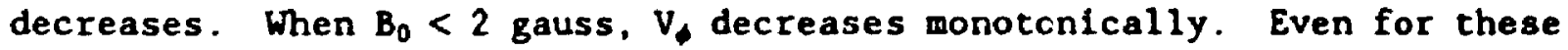
case;, $V_{0}$ is still higher than the azimuthal velocity for angular momentum conservation ( curve 5 of Figure 4-4). The distribution of the azimuthal component of the magnetic field is given in Figure 4-5. The absolute

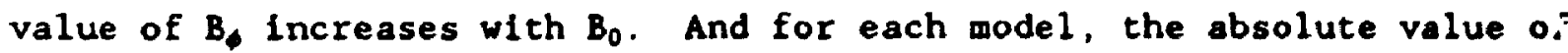
$B_{\phi}$ decreases monotonically with distance. When $B_{0}-20$ gauss, $\left|B_{\phi}\right|$ at the surface is 250 gauss, comparable to the upper limit quoted by Barker (1987). 


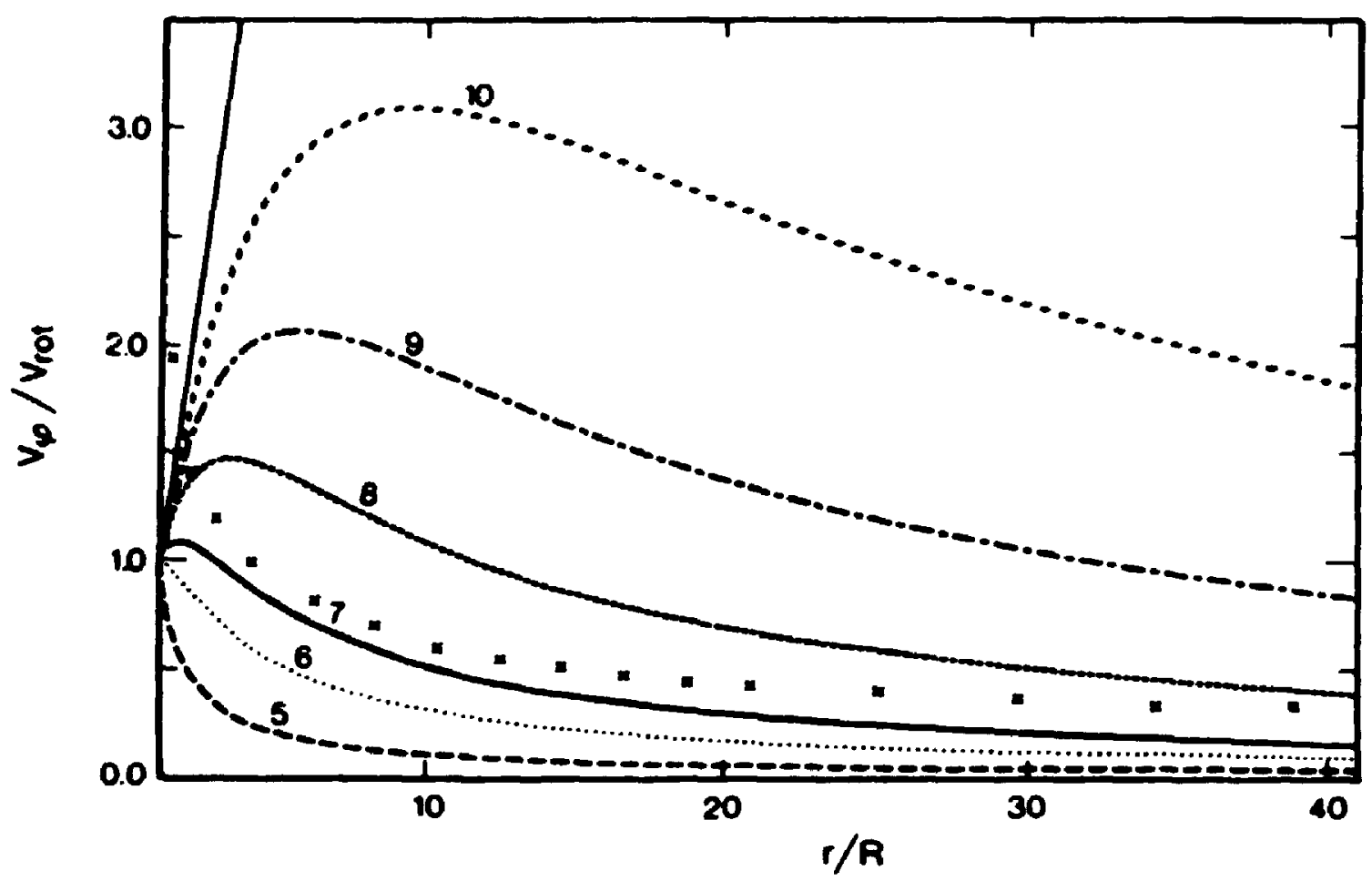

Figure 4-4. The distribution of the azlauthal velocity for the nodels glven in Table 4-2. The numbers Indicate the corresponding nodel numbers in Table 4.2 . The stralght line is the aziouthal velocity distribution for solid body rotation. The neaning of curve 5 is the sane as that in Flgure 4-1. The curve indicated by crosses is the ratio of the local escape speed to the stellar rotation speed. 


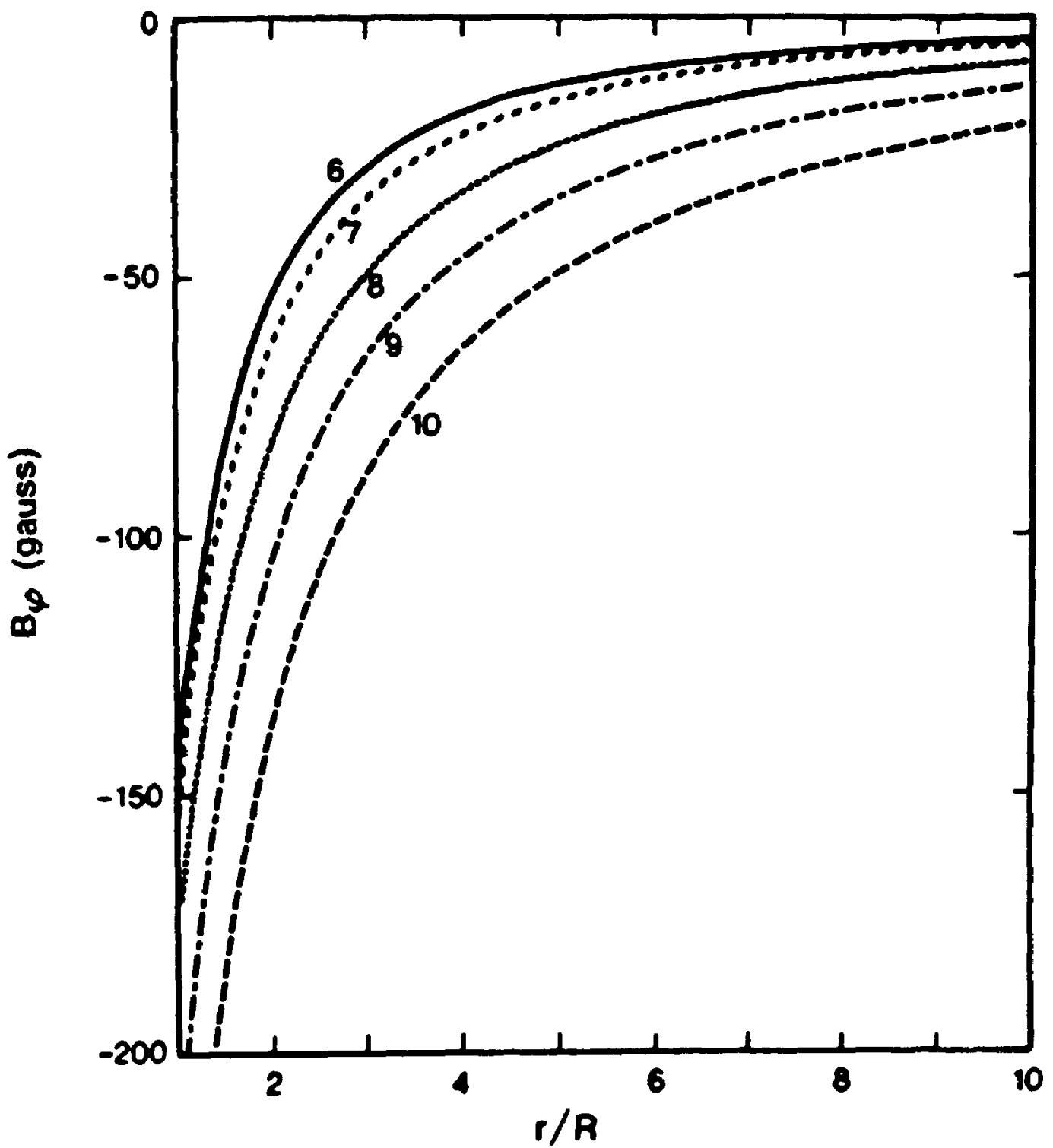

Figure 4-5. The distribution of the azinuthal component of the magnetic field for the model: given in Table 4-2. The numbers indicate the corresponding model numbers in Table 4-2. 


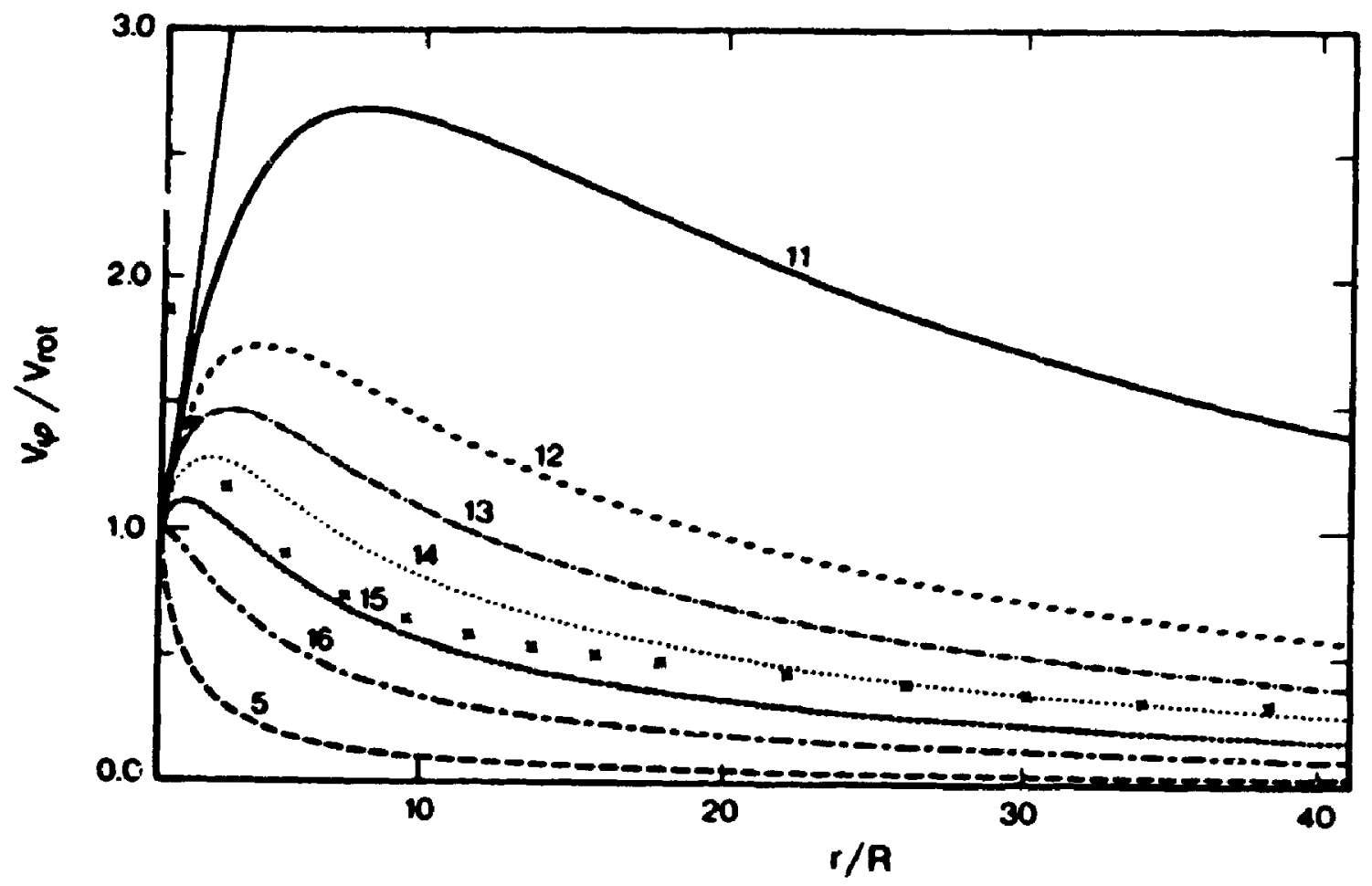

Figure 4-6. The distribution of the azlouthal veloclty for the models given In Iable 4-3. The numbers Indicate the corresponding nodel numbers in Table 4.3 . The strafght line is the azinuthal velocity discribution of solid body rotation. The neasing of Curve 5 is the sane as that in Figure 4-1. The curve indicated by crosses is the rat1o of the local escape speed to the stellar rotation speed. 


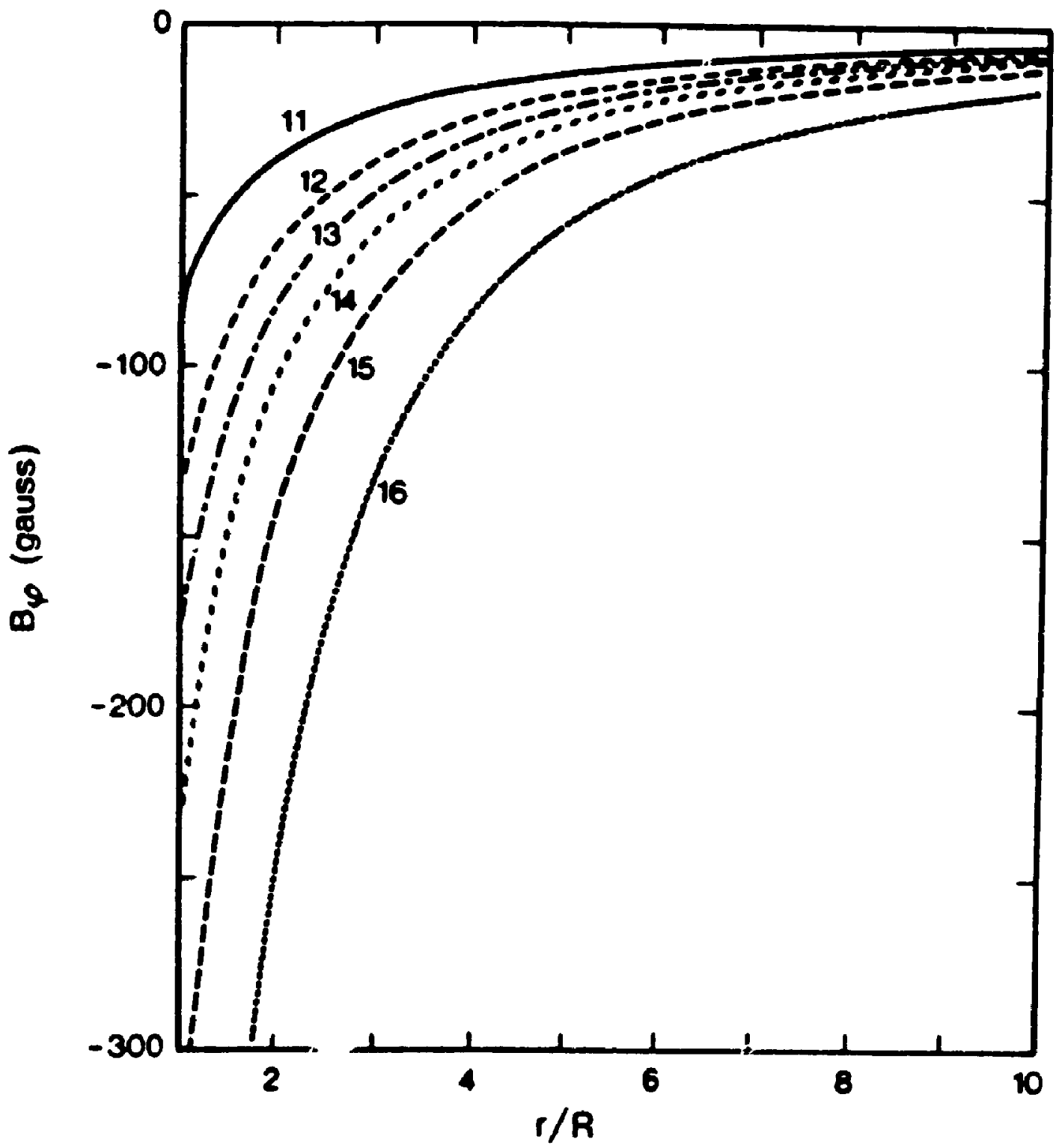

Figure 4.7. The distribution of the azimuthal component of the magnetic field for the models given In Table 4-3. The numbers indlcate the corresponting model numbers in table 4.3. 
The results for the models with $m-J .7$ and $B_{0}-5$ gauss but differe $\because$ mass loss rate are given in Table $4-3$ and Figures 4-6 and 4-7. As the $\cdots . . . s$ loss irte increases, both the Alfven radius and the total angular momentum per unit mass decrease. The distribution of azimuthal velocity depends on the mass loss rate. The smaller is the mass loss rate, the higher is the azimuthal velocity. When the mass loss rate $\leq 10^{-7}$ $M_{0} y^{-1}, V_{0}$ increases to its maximum at about the Alfven point and then decreases. When the mass loss rate is larger than $10^{-7} \mathrm{M}_{0} y^{-1}$, the azimuthal velocity decreases munotonicaliy with increasing distance. Because of the transfer of the angular momentum, the azimutha] velocity of all models is much higher tinan that for the case of angular momentum conservation (curve 5 of Figure 4-6). The distribution of the azimuthal component of the magnetic field is illustrated in Figure 4-7. The absolute value of $B_{\phi}$ increases with increasing mass loss rate and for each model it decreases monotonically with the distance.

The results for the models with $B,-5$ gauss and $\dot{M}=10^{-2} M_{\odot} y^{-1}$ but different velocity index $m$ are illustrated in Table 4-4 and Figures 4-8 and 4-9. The larger is the value of $m$, the steeper is the radial velocity curve. As $m$ increases, both the Alfven radius and the total angular momentum per unit mass decrease. For all $\mathrm{m}$ in the range of interest, the azimuthal velocity curves have similar behaviour. They all in:rease to a maximum and then decrease. The azimuthal velocity corresponding to smaller m reaches a higher miximum and decreases less rapidly. Again, for comparison. the azimuthal velocity for angular momentum conservation is plotted (curve 5 of Figure 4-8). The distribution of the azimuthal component of the magnetic field is given in Figure 4-9. The absolute value of $B_{0}$ decreases with increasing $m$. And for earh model, the absclute 


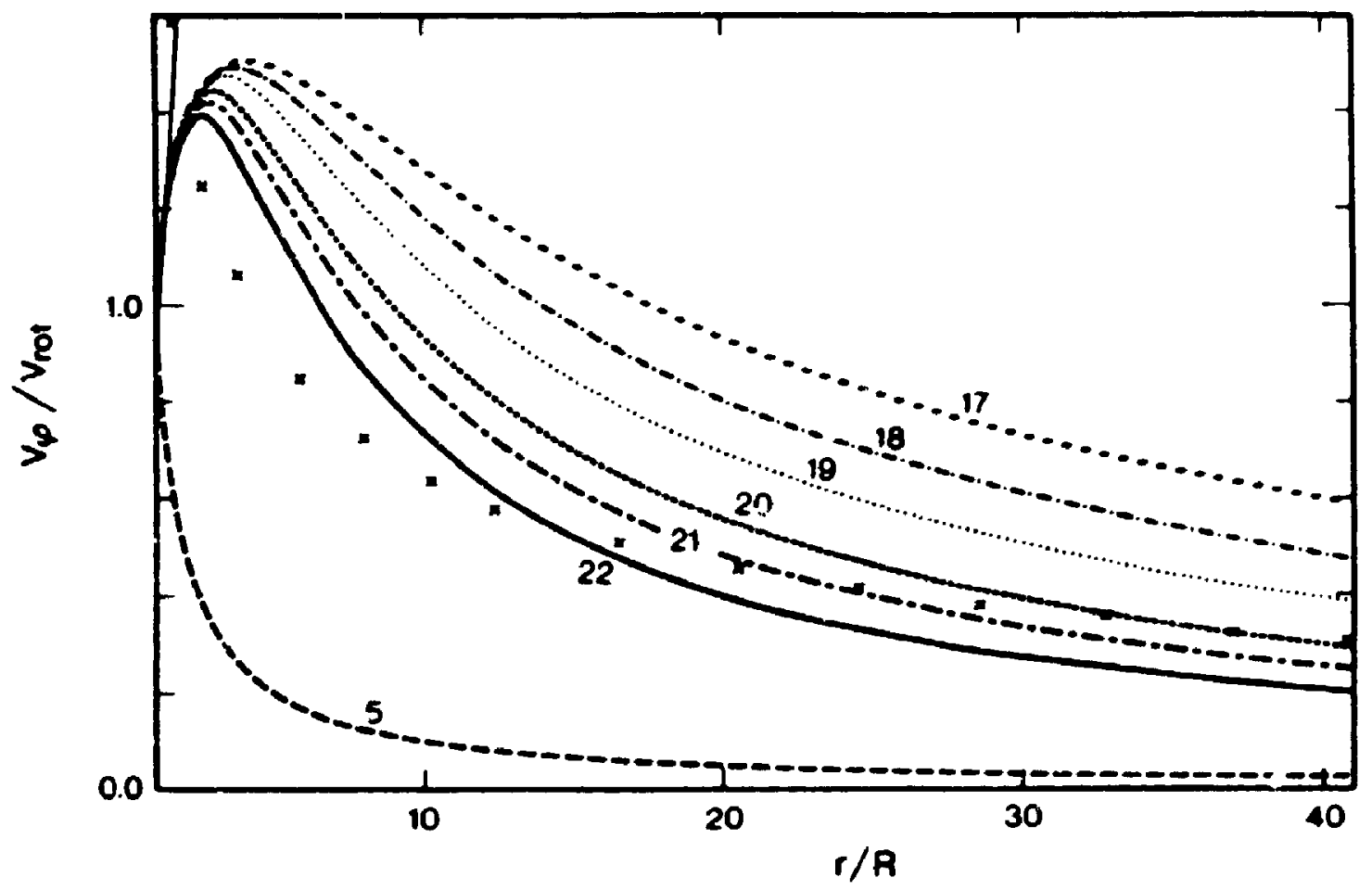

Figure 4-8. The distribution of the azlmuthal velocity for the models given in Table 4-4. The numbers Indicate the corresponding model numbers in Table 4.4. The stralght line is the azimuthal veioctsy distribution for solld body rotation. The meaning of Curve 5 is the same as that in Figure 4-1. The curve indicated by crosses is the ratio of the local escape speed to the stellar rotation speed. 


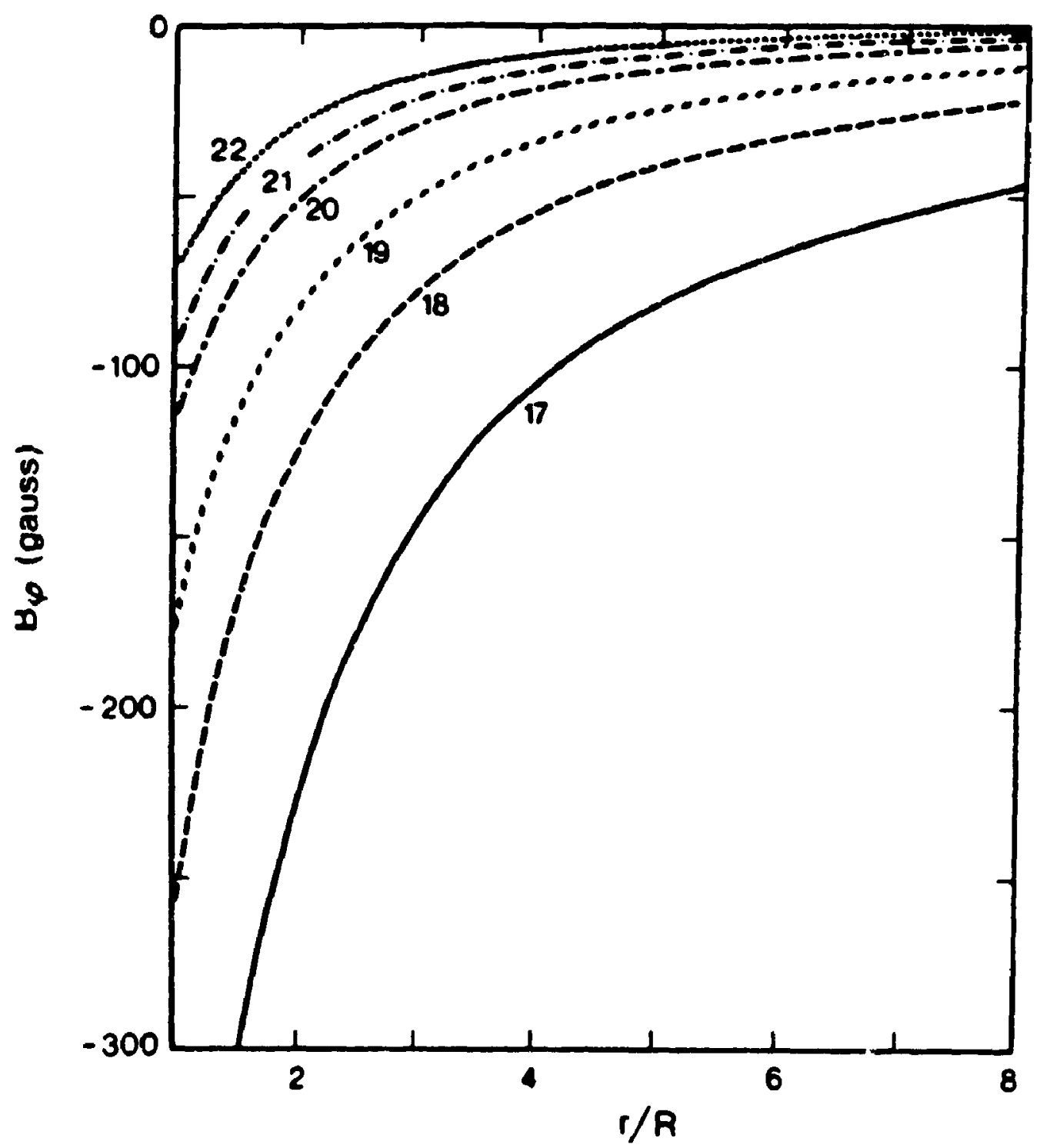

Figure 4-9. The distribution of the azimuthal component of the magnetic field for the models given in Table 4-4. The numbers indicate the corresponding model numbers in Table 4-4. 
value of $B_{\phi}$ decreases monotonically with distance.

The azimuthal velocity distribution for solid body rocation is also given in Figures $4-4,4-6$ and 4-8 (the straight line in each case). For all models considered, the azimuthal velocity distribution is below that of solid body rotation, in contrast with the results for a $\beta$ velocity distribution.

\subsection{WHAT $F_{x}$ IS NEEDED?}

I have shown how a weak radial magnetic field influences the azimuthal velocity and the azimuthal magnetic fleld of a slowly expanding wind. The magnetic field also influences the radial motion of the wind in two ways. First, the magnetic field exerts a force on the materiol in the radial direction. And second, the centrifugal force is enhanced as the result of the increase of the azimuthal velocity. The expression for the radial component of the magnetic force is given by equation (4-5). The force is given in Figure 4-10 for two models, models 15 and 16 . I note that the force is several orders of magnicude smaller than boch gravity and the centrifugal force ( see Figures 2-11 and 2-12). This implies that the radial component of the magnetic force of a weaic field does not make a significant contribution to the driving of the wind in the radial direction.

By inverting the equation of motion, the same approach that I used in Chapter 2, I can obtain the unknown force if one is actually required, in addition to those known forces arising from gravitation, rotation, the gas pressure gradient and the magnetic field, required to produce a radial component of velccity having an assumed functional form. The unknown force $\ddot{r}_{x}$ as well as the forces arising from gravity, rotation and gas 


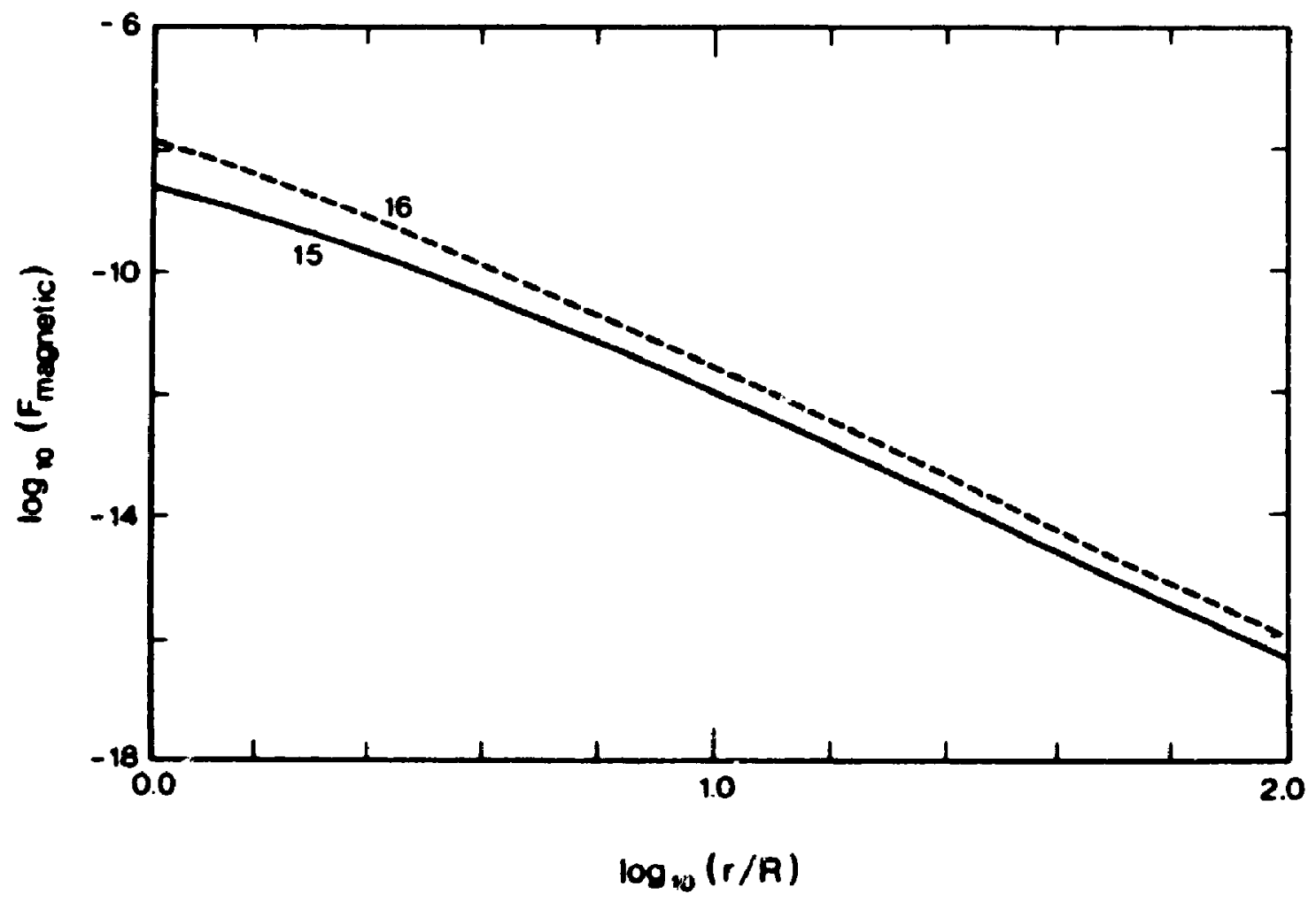

Figure 4-10. The radial component of the magnetic force for models 15 and 16. 


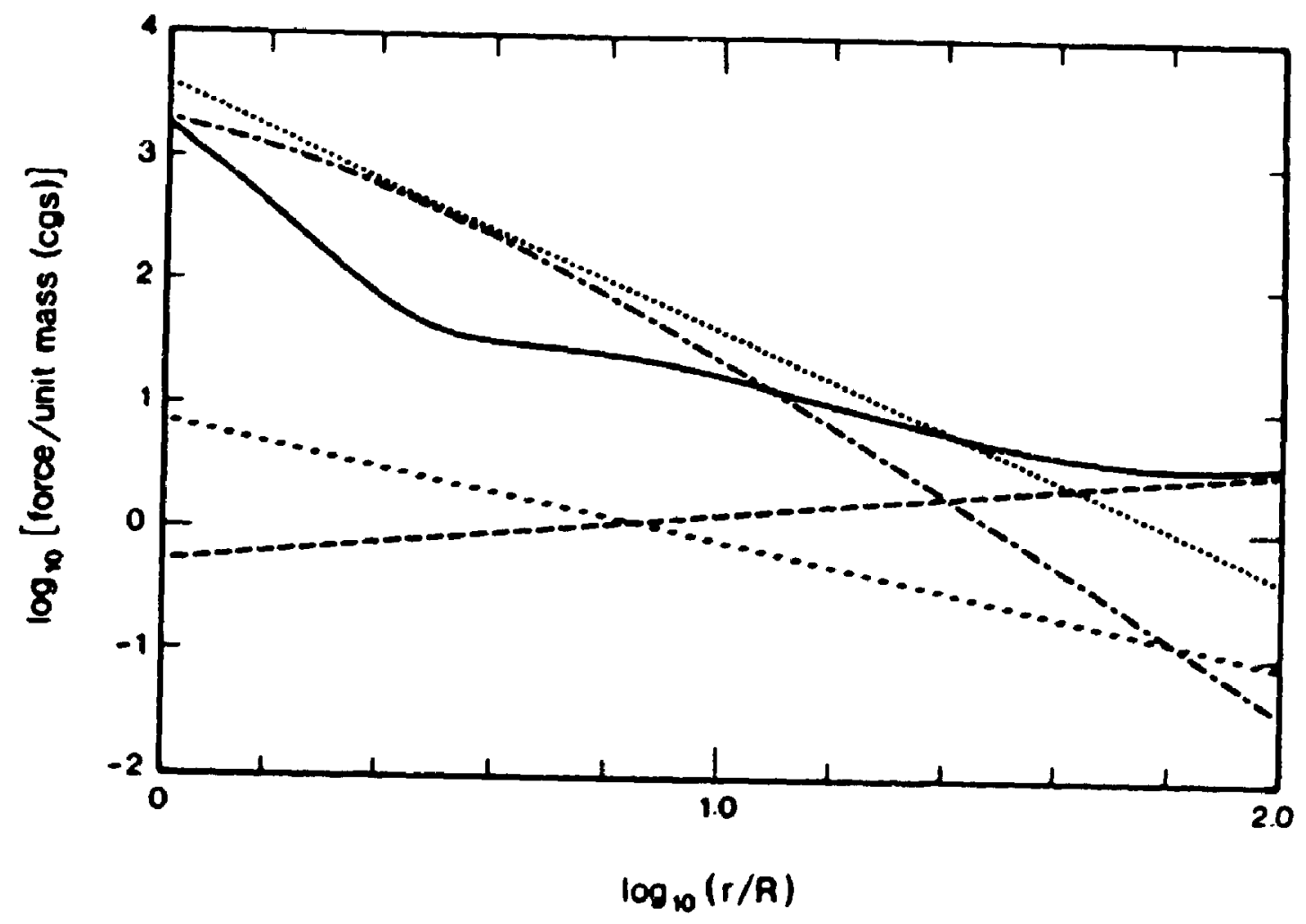

Figure 4-11. The dependence of the force terus on $r / R$ for model 16 . The solid curve is $F_{2}$, the unknown force; the short dashed curve is $F_{8}$, the gravitational force; the long dashed curve is the inertal terd VdV/dr; the long dashes separated by shorter dashes represent the centrlfugal term; and the short dashes separated by larger spaces represent the gas pressure term. 
pressure gradient for model 16 are given in Figure 4-11. I do not include the radial component of the magnetic force in the graph since it is several orders of magnitude smaller than gravity. Because of the transfer of the angular momentum from the magnetic field, the azimuthal velocity is increased, therefore the centrifugal force is much larger than that when angular momentum conservation of material is considered. In the case of angular momentum conservation, the centrifugal force decreases more rapidly than does the gravity. In contrist, the centrifugal force now decreases less rapidly than does gravity. The centrifugal force almost balances gravity. As a result, the additional force required to produce the assumed radial velocity law is smaller than that when no magnetic field is present.

Various forces for model 15 are given in Figure 4-12. In this case, which is of academic interest only because of the parameters chosen, the azimuthal velocity is so large (see curve 15 of Figure 3-6) that the centrifugal force exceeds gravity in the region between 1.6 to 15 stellar radil. If I were to formally assert that the assumed form of radial velocity is correct, the unknown for would be negative, i.e., the force would act in the same direction as gravity does. 


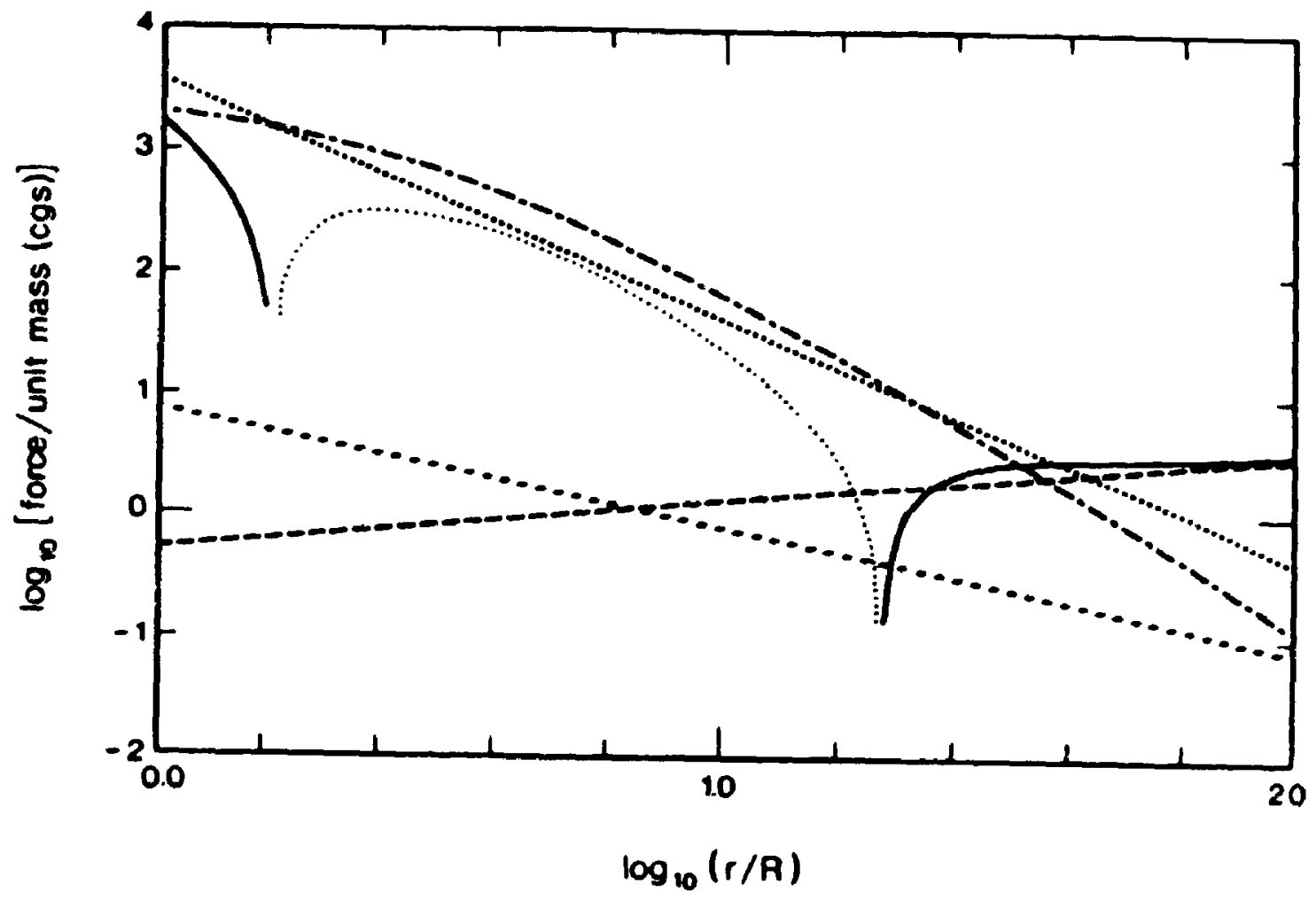

Flgure 4-12. The dependence of the force terms on $r / R$ for model 15 . The meanling of each curve is the same as that in figure 4.11 except that the solid line now is separated by dots, which shows the range of $r$ In which the force is polnting inwards. 
CHAPTER 5. WINDS DRIVEN BY OPTICALLY THIN LINES

\subsection{INTRODUCTION}

In Chapter 2, I discussed the form of the unknown driving force/forces, $F_{x}$, of Be star envelopes. According the form of $F_{x}$, I excluded radiation pressure due to optically thick 1 ines as an important driving force for Be star envelopes. I then discussed several other mechanisms which could possibly explain the form of $F_{x}$. Two of then are the presence of a weak magnetic field and the radiation force due to the optically thin lines.

In Chapter 4, I then investigated how a weak radial magnetic field $\left(B_{0} \sim 10\right.$ gauss) influences the dynamics in the equatorial plane of the envelopes of Be stars. My investigation shows that in a slowly expanding envelope, a weak magneic field can influence the rotational velocity distribution quite effectively by transferring angular momentum from the magnetic field to the material. The radial component of the magnetic field forse is negligible for the strength of the magnetic field considered. But the centrifugal force is enhanced due to the transfer of angular momentum to the material. For some cases the centrifugal force is so large that it can halance gravity.

In this chapter, I am going to discuss another possible mechanism that I proposed in Chapter 2, i.e., the radiation force due to optically thin lines or, in short, the weak line force.

The importance of a weak line force in driving stellar winds has been discussed by Lamers (1986). After investigating the dynamics of the wind of $P$ Cygni, he concluded that the wind was best explained by radiation force arising from a large number of optically thin lines. 
Although no detalled calculation has ever been done to show how large the weak line force could be, the presence of a very large number of optlcally thin lines in IUE spectra for Be stars provides evidence that this force could play an important role in driving the envelopes of Be stars.

The remainder of this chapter is organized as follows. In $\$ 5.2$ I discuss the assumptions about the weak line force and derive the form of the weak line force; in $\$ 5.3$ I consider the equation of motion in the equatorial plane when the weak line force is considered; in $\$ 5.4 \mathrm{I}$ discuss the dynamical equation analytically; in $\$ 5.5$ I calculate the wind model driven by the weak line force for a Be star, $\gamma$ Cas; and In \$5.6, I discuss the application of my results to the envelope of Be stars.

\subsection{THE FORCE DUE TO OPTICALLY THIN LINES}

The radiation force due to an optically thin 1 ine can be expressed as

$$
f_{-}=\frac{\kappa_{1} F_{1}}{c}-\frac{\kappa_{1} L_{2}}{4 \pi c r^{2}}
$$

where $c$ is the speed of light; $\kappa_{1}$, the integral of line opacity over the line profile; $F_{3}$, the monochromatic flux per unit wavelength at the wavelength of the weak 1 ine; and $L_{1}$, the monochromatic luminosity per unit wavelength of the star at the corresponding wavelength.

If the total number of optically thin lines is $\mathrm{N}$, the force due to all weak lines will be

$$
F_{\nabla}-\sum^{n} \frac{\kappa_{1} L_{1}}{4 \pi c r^{2}}
$$


where $\Sigma$ sums over all $N$ weak lines. If I express the line opacity $\kappa_{1}$ in terms of the electron scattering coefficient, 0 , i.e.,

$$
\kappa_{1}-\delta_{1} \sigma
$$

the force $F_{\text {v }}$ can be expressed as

$$
F_{v}=\frac{a}{4 \pi c r^{2}} \sum^{u} \delta_{1} I_{2}
$$

I introduce an average

$$
<\delta>-\frac{1}{N} \sum^{n} \frac{L_{1}}{L_{0}} \delta_{1}
$$

where $L$. is the total luminosity of the star. The average $\langle\delta\rangle$ is an average of opacity of all weak lines weighted by local monochromatic luminosity. Equation $(5-4)$ becomes

$$
F_{-}-\frac{o L, N<\delta>}{4 \pi c r^{2}}
$$

Using $\Gamma-\frac{\sigma L .}{4 \pi c G M}$, equation $(5-6)$ becomes

$$
F_{-}=\frac{G M \Gamma}{r^{2}} N\langle\delta>
$$

I define

$$
W-N<\delta>\frac{\Gamma}{\Gamma-\Gamma}
$$

In general, the total number of weak 1ines, $N$, is a function of distance since it depends on the local excitation and lonization equilibrium. And so is the average opacity $\langle\delta\rangle$. In this work I am not going to investigate the dependence of $N$ and $\langle\delta\rangle$ on $r$ from first principles. Instead I assume that the product of $N$ and $\langle\delta\rangle$ is a Eunction of $r$, therefore, 


$$
W(r)-W-N<\delta>\frac{\Gamma}{I-\Gamma}
$$

Equation (5-7), the expression for the force due to the optically thin Iines, becomes

$$
F_{\nabla}-\frac{G M(1-\Gamma)}{r^{2}} H(r)
$$

The physical meaning of $W(r)$ can be seen from equation (5-10). It is the ratio of the force due to the optically thin lines over the gravitational term.

\subsection{THE DYNAMICS IN THE EQUATORIAL PLANE}

\subsubsection{The Equation of the Motion}

As in Chapter 2, the circumstellar envelope is assumed to be symmetric about the rotation axis and the equatorial plane. The equation of motion describing the dynamics in the radial direction in the equatorial plane is given by Equation (2-1). In this chapter, I consider the force due to optically thin lines as the driving force. The unknown force there, $F_{x}$, is replaced by the weak line force $F_{w}$. Equation (2-1) can be rewritten as

$$
v_{x} \frac{d v_{x}}{d r}-F_{q}+F_{p}+F_{c}+F_{v} \text {. }
$$

where $F_{q}, F_{p}$, and $F_{c}$, same as in Equation (2-1), are the gravitational force per unit mass reduced for electron scattering, the force due to the gas pressure gradient, and the centrifugal force respectively. And $F_{*}$ is the force due to the optically thin lines.

If I substitute the expressions for each term in equation (5-11), it becomes 


$$
\left(1-\frac{a^{2}}{v_{r}^{2}}\right) v_{\mathrm{r}} \frac{d v_{\mathrm{s}}}{d r}=-\frac{G M\left(1-\Gamma_{\bullet}\right)}{r^{2}}+\frac{2 a^{2}}{r}-\frac{d a^{2}}{d r}+\frac{v_{t}^{2}}{r}+\frac{G M\left(1-\Gamma_{\bullet}\right)}{r^{2}} H(r),
$$

where a is the sound speed, and other terms have their normal meaning. In the following discussion I will only consider an isothermal wind, so the term $\frac{d a^{2}}{d r}$ vanishes. Equation (5-12) becomes

$$
\left(1-\frac{a^{2}}{v_{z}^{2}}\right) v_{x} \frac{d v_{x}}{d r}--\frac{: M\left(1-\Gamma_{\bullet}\right)}{r^{2}}+2 \frac{a^{2}}{r}+\frac{v_{\phi}^{2}}{r}+\frac{G M\left(1-\Gamma_{\bullet}\right)}{r^{2}} W(r)
$$

Because of the assumption of an isothermal wind, the left-hand side of equation (5-13) is the function of $v_{z}$ and $d v_{z} / d r$ only. It can be integrated in terms of $x$ to obtain $1 / 2 v_{z}{ }^{2}-a^{2} l n v_{z}$ plus a constant. On the right-hand side, the first and second terms are obviously a function of $r$ only. According to my assumption about $W(r)$, the forth term is also a function of $r$ only. The behavior of the third term depends on the form of $v_{4}$. In the following, I assume that it is either given by angular momentum conservation or is Kepleriar. In either case, the third term of equation (5-13) is the function of $r$ only. So the right-hand side can be integrated in terms of $r$ to give:

$$
\frac{1}{2} v_{z}^{2}-a^{2} \ln v_{r}-\frac{G M\left(1-\Gamma_{0}\right)}{r}+a^{2} \ln r+R I(r)+W I(r)+C
$$

where $C$ is the constant of integration and $R I(r)$ and $W I(r)$ are the integral of the rocational term and the weak line force term, respectively. Their expressions are

$$
R I(r)-\int \frac{v_{i}^{2}}{r} d r,
$$


and

$$
W I(r)-G M(1-\Gamma) \int \frac{W(r)}{r^{2}} d r
$$

The characteristic of equation (5-13) is similar to that of the solar wind equation (Parker 1958). The solution I an interested in is the one that starts from a subsonic speed at the surface of the star and passes through the sonic point, at which both sides of equation (5-13) vanish, and continues to increase to supersonic speeds beyond the sonic point.

To solve equation (5-14) numerically, one has to evaluate the constant of integration. I choose the sonic point as the reference point to determine its value. At the sonic point, the left-hard side of equation (5-12) vanishes. So by setting the right-hand side of the equation to be zero, I can determine the sonic point:

$$
2 a^{2} r_{a}+v_{b}^{2}\left(r_{e}\right) r_{a}-G M(1-\Gamma)+G M(1-\Gamma) W\left(r_{e}\right)-0
$$

Once the constant of integration in equation (5-14) is determined, the velocity can be obtained at each $r$ by solving equation (5-14). By substituting the values of speed and radius at the sonic point, I have

$$
C-\frac{1}{2} a^{2}-a^{2} \ln a-\frac{G M(1-\Gamma)}{r_{c}}-2 a^{2} \ln r_{c}-R I\left(r_{c}\right)-W I\left(r_{c}\right)
$$

If I use the dimensionless distance

$$
x-\frac{r}{R}
$$


and velocity

$$
y=\frac{v_{x}}{a}
$$

equation (5-13) can be written as

$$
\frac{1}{2} y^{2}-1 n y+H-0
$$

where

$$
\begin{gathered}
H=-(E+D), \\
E-\frac{y_{c x i}^{2}}{x}+2 \ln x x+R I(x)+W I(x), \\
D=\frac{1}{2}-\frac{y_{c r i}^{2}}{x_{c}}-2 \ln x_{c}-R I\left(x_{c}\right)-W I\left(x_{c}\right)
\end{gathered}
$$

and equation (5-17) becomes

$$
2 x_{a}^{2}-y_{b}^{2} x_{a}+y_{b}^{2} W\left(x_{a}\right)+y_{b}^{2} x_{a}^{2}-0
$$

where $y_{b}$ is the dimensionless break-up speed

$$
y_{b}=\frac{V_{b s o a k-u p}}{a}-\frac{1}{a}\left(\frac{G M(1-\Gamma)}{R}\right)^{1 / 2}
$$

\subsubsection{The Determination of $y_{*}$ and $R I(x)$}

To solve the equations I need to know the form of $y_{0}$, the dimensionless rotational velocity and $R I(x)$, the integral of $y_{\text {, defined in }}$ equation (5-15). In my discussion, I assume the dependence of the rotational velocity on $r$ either is given by angular momentum conservation or is Keplerian. For angular momentum conservation

$$
y_{\downarrow}-\frac{y_{\text {rot }}}{x}
$$




$$
R I(x)=-\frac{y_{10 t}}{2 x}
$$

where $y_{r o t}$ is dimensionless rotational velocity:

$$
y_{\text {rot }}-\frac{v_{\text {rot }}}{a}
$$

On the other hand, if the rotational velocity is Koplerian, I will have

$$
y_{+}-\frac{y_{1 o t}}{\sqrt{x}}
$$

and

$$
R I(x)=-\frac{y_{\text {rot }}^{2}}{x}
$$

In the following, a Keplertan rotation velocity is assumed u.nless it is specified otherwise.

5.3.3. The Determination of $W(x)$ and $W I(x)$

The weak line force factor $W(r)$ is defined by equation (5-8). It may have a very complicated dependence on $r$. At present, I am not able to find its form from first principles. And since it is not my intention in this work to do the detailed computation of $W(r)$, I will assume $W(r)$ to have a simple dependence on $r$. I will adjust free parameters so that a reasonable radial velocity distribution will be produced. I assume

$$
W(x)-\eta x^{\varepsilon}
$$

where $\eta$ and $c$ are constants. Under this assumption, the weak line force will have the same dependence upon $r$ as I proposed for $F_{x}$ In Chapter 2 (see 
equations 2-11 and 5-9). In equation (5-32), 7 weasures the strength of the force due to the optically thin lines at the surface of the star, and c measures the dependence of the force on $r$. For $W(r)$ described in equation $(5-32)$,

$$
W I(x)=-\frac{\eta}{I-c} \frac{y_{\text {eri }}^{2}}{x^{1-c}}
$$

5.4. Analytic Analysis of the Dynamical Equation

5.4.1. The Restriction for the Values of $c$ and $\eta$

Substituting equation (5-30), the Keplerian velocity law, and equation (5-32), the weak line force factor, into equation (5-25), which determines the sonic point, I have

$$
2 x_{a}+y_{b}^{2} \eta x_{a}^{e}+y_{b}^{2}\left(\lambda^{2}-1\right)-0
$$

or in another form

$$
\frac{2}{y_{b}^{2}} x_{-}+\eta x_{e}^{e}-1-\lambda^{2}
$$

If I define

$$
f(x)-\frac{2}{y_{b}^{2}} x
$$

and

$$
g(x)-\eta x^{e}
$$

equation (5-35) becomes

$$
f(x)+g(x)-1-\lambda^{2} \text {. }
$$


For a star the value of $\lambda$, the ratio of rotational velocity of the star over the break-up velocity, is a constant, which $I$ assume to be 0.7 in my research. The function $f(x)$ is a linear function of $x$ with a slope of $2 / y_{b}{ }^{2}$. Once the star is given, $y_{b}$, and therefore, $f(x)$ is given. For a star like $\gamma$ Cas, $y_{b}{ }^{2}-1500$, the slope, $2 / y_{b}{ }^{2}-0.0013 \ll 1$. The function $g(x)$ is power law function with index $c$. The value of $g(x)$ at given $x$ depends both on $\eta$ and $\epsilon$. The behavior of $f(x)$ and $g(x)$ is illustrated in Figure

5-1. Since the slope, $2 / y_{b}{ }^{2}$, of function $f(x)$ is very small, the dependence of $f(x)$ on $x$ is very hard to see. To overcome this, I enlarge the slope artificially by a factor of ten. The value of $1-\lambda^{2}$ is illustrated as a straight horizontal line in Figure 5-1. I can be shown that for a given $x$, if $f(x)+g(x)<1-\lambda^{2}, x<x$; if $f(x)+g(x)>1-\lambda^{2}$, $x$ $>x_{0}$. Since $g(x)$ depends on the value of $\eta$ and $\epsilon$, the sonic point will depend on the values of $\eta$ and $\epsilon$ for a given rotational rate.

Since I want the solution of the wind to start from a subsonic speed and pass through the sonic point and then reach supersonic speed, the sonic point should be outside the star, 1.e., $x_{2}>1$. From Figure 5-1 I san sec that the condition of $x_{0}>1$ requires

$$
\frac{2}{y_{b}^{2}} x+\eta x^{e} b_{x-1}<1-\lambda^{2}
$$

or

$$
\eta<1-\lambda^{2}-\frac{2}{y_{b}^{2}}
$$

Compared with 1 and $\lambda^{2}$, the third term is wuch smaller and can be ignored.

The region below the sonic point is a subsonic region. For Be 


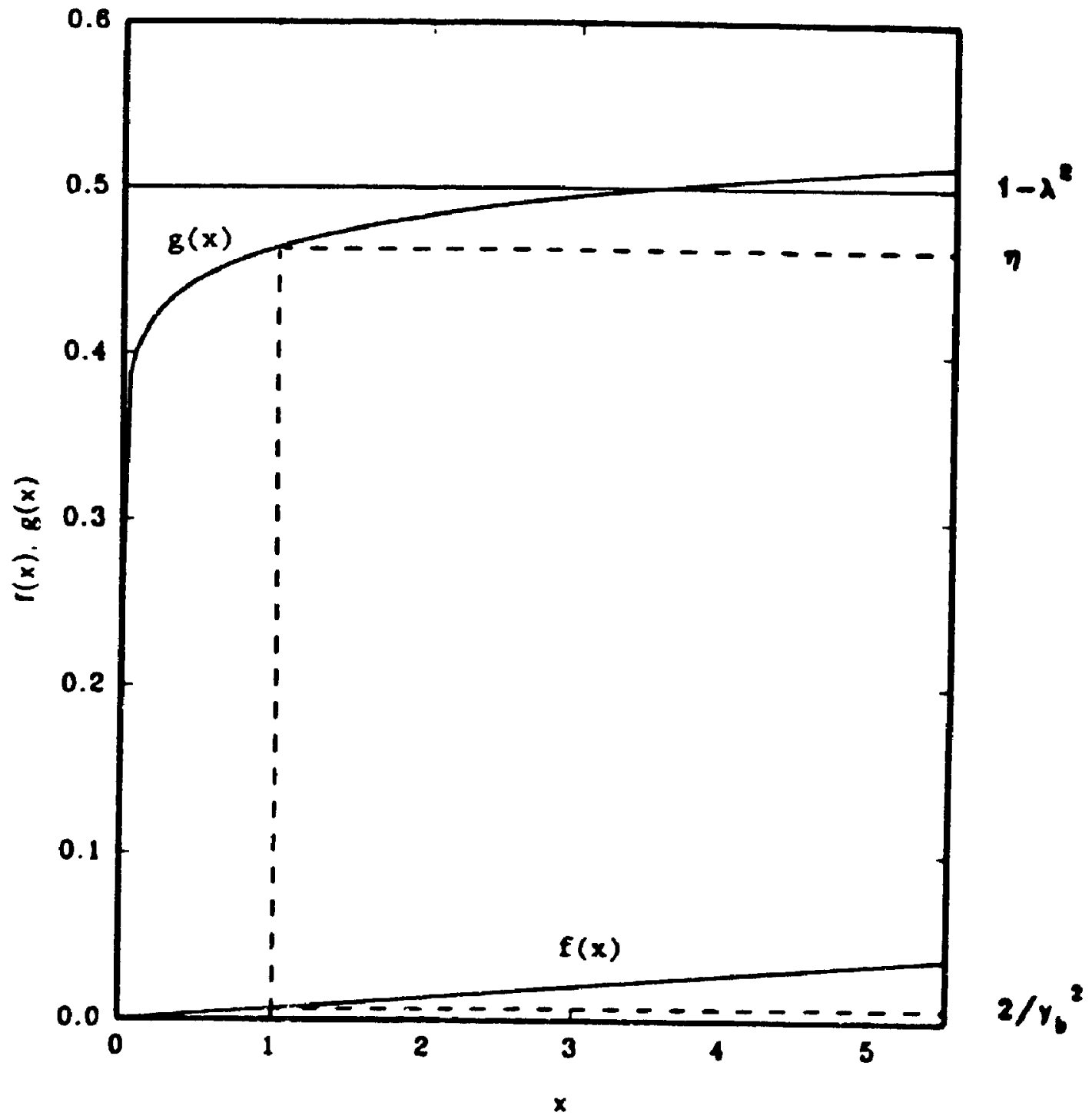

Figure 5-1. The behaviour of $f(x)$ and $g(x)$. The slope of $f(x)$ has been enlarged a factor of 10 for 1llustration. 
stars, there are no direct observations showing how big this region could be. However, if it is very big.e.g., 100R in radius, it is not likely that we are able to see the surface of the stars because of the high column density in the subsonic region. So it is reasonable to assume an upper limit for the size of the subsonic region. Assuming the maximum value of the radius of the sonic point is $x_{n}$. I can find the relation:

$$
\frac{2}{y_{b}^{2}} x+\eta x^{e} b_{x-x_{m}} \geq 1-\lambda^{2}
$$

or

$$
\eta x_{i}^{e} \geq 1-\lambda^{2}-\frac{2}{y_{b}^{2}} x_{i}
$$

The equal sign in equation $(5-42)$ holds when $x_{*}=x_{3}$. In Figure 5-2, I summarize the restriction on the values of $c$ and $\eta$ from the discussion above. From the requirement of $x_{*}>1$, the valid value of $\eta$ is below the straight line $\eta-1-\lambda^{2}-2 / y_{b}{ }^{2}$. On the other hand, from the requirement of $x_{1} \leq x_{n}$, the values of $c$ and $\eta$ should be above the curve corresponding to equation (5-42). In the figure, the curves corresponding to $x_{\mathbf{m}}-2,3,5$ and 10 are given.

\subsubsection{The Terminal Velocity}

The terminal velocity of a wind is a fixed value the wind speed approaches when $x$ approaches infinity. To see if the wind determined by equation (5-21) has a terminal velocity, I differentiate equation (5-21) to have:

$$
\left(y-\frac{1}{y}\right) \Delta y-\left[-\left(1-\lambda^{2}\right) \frac{y_{b}^{2}}{x^{2}}+\eta \frac{y_{b}^{2}}{x^{2-e}}+\frac{2}{x}\right] \Delta x,
$$




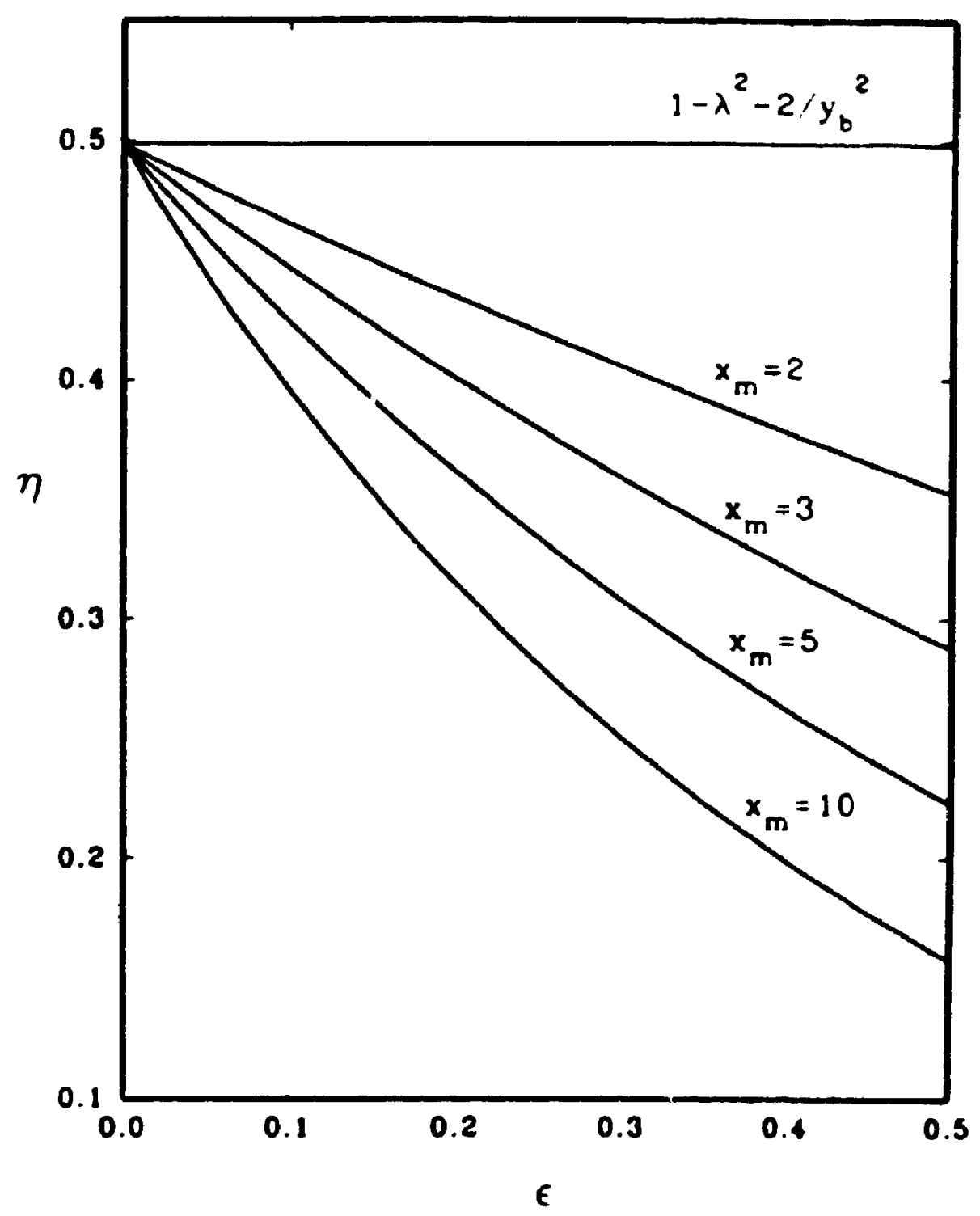

Figure 5-2. The 11 mitation on and $\eta$. The requirement that $x_{c}$ should greater than 1 Indicates that the value of $\eta$ should lie below the horizontal 1 ine. And the requirement that $x_{*}$ be smaller than $x_{0}$ indicates that the values of $c$ and $\eta$ should lie above the corresponding curve. 
or

$$
\Delta y=\frac{1}{y-\frac{1}{y}} h(x) \Delta x
$$

where $h(x)$ is a function with the same order of magnitude as $1 / x$. In equation (5-44), when $x \rightarrow \infty, \Delta y \rightarrow 0$. In other words, $y$ approaches a fixed value when $x$ increases to a large number.

\subsubsection{Intial Velocity}

The initial velocity, $y_{0}$ is determined by setting $x-1$ in equation (5-21). I have

$$
\frac{1}{2} y_{0}^{2}-\ln y_{0}-y_{b}^{2}\left[\left(1-\lambda^{2}\right)\left(1-\frac{1}{x_{0}}\right)-\frac{\eta}{1-\epsilon}\left(1-\frac{1}{x_{0}^{1-e}}\right)\right]-2 \ln x_{0}+\frac{1}{2}
$$

\subsection{VELOCITY DISTRIBUTION CALCULATIONS}

The procedure for the calculation is the following: I choose values of $\epsilon$ and $\eta$ and solve equation (5-25) to obtain the sonic point, $x_{a}$. Then I solve equation (5-21) to obtain $y$ for each $x$. In the following the calculation is for a star like $\gamma$ Cas: $M-16 M_{0}, R-10 R_{0}, T-25,000 \mathrm{~K}$, $L-5.4 \times 10^{4} L_{0}$.

Since both $\eta$ and $c$ are free parameters, I first choose $\epsilon$ values and then calculate the wind model for different $\eta$ values. Models for $c=$ $0.01,0.05,0.10,0.20$ and 0.30 are given in Tables 5-1,5-2,5-3, 5-4 and 5-5, respectively. In the tables, column 1 and column 2 give $\epsilon$ and $\eta$ values respectively. Column 3 gives the sonic point in dimensionless variables. Column 4 gives initial velocities in $\mathrm{km} / \mathrm{s}$. Column 5 gives $V_{100}$, the velocity at 100R. Although $v_{100}$ id not the terminal velocity, $V_{0}$, 
TABLE 5-1

MODELS FOR $€-0.01$

\begin{tabular}{cccccc}
\hline$c$ & $\eta$ & $x_{c}$ & $V_{0}(\mathrm{~km} / \mathrm{s})$ & $v_{100}(\mathrm{~km} / \mathrm{s})$ & $R_{\mathrm{c}}(R)$ \\
\hline 0.01 & 0.495 & 1.71 & 2.6 & 69.7 & 4.3 \\
0.01 & 0.490 & 3.16 & 0.04 & 59.6 & 7.2 \\
0.01 & 0.480 & 7.46 & $<10^{-6}$ & 47.9 & 13.5 \\
\hline
\end{tabular}

TABLE 5-2

MODELS FOR $<-0.05$

\begin{tabular}{cccccc}
\hline$c$ & $\eta$ & $x_{c}$ & $V_{0}(\mathrm{~km} / \mathrm{s})$ & $V_{100}(\mathrm{~km} / \mathrm{s})$ & $R_{\mathrm{t}}(R)$ \\
\hline 0.05 & 0.495 & 1.15 & 6.8 & 127.6 & 2.9 \\
0.05 & 0.490 & 1.39 & 1.3 & 117.5 & 3.4 \\
0.05 & 0.480 & 2.02 & 0.004 & 100.1 & 4.5 \\
0.05 & 0.460 & 4.20 & $<10^{-6}$ & 74.7 & 9.1 \\
0.05 & 0.440 & 8.18 & $<10^{-6}$ & 57.5 & 15.2 \\
\hline
\end{tabular}


Table 5-3

MODELS FOR $<-0.10$

\begin{tabular}{cccccc}
\hline$c$ & $\eta$ & $x_{c}$ & $V_{0}(\mathrm{~km} / \mathrm{s})$ & $V_{100}(\mathrm{~km} / \mathrm{s})$ & $R_{\mathrm{b}}(\mathrm{R})$ \\
\hline 0.10 & 0.495 & 1.07 & 86 & 178.7 & 2.8 \\
0.10 & 0.490 & 1.18 & 3.2 & 170.4 & 3.1 \\
0.10 & 0.480 & 1.45 & 0.1 & 155.0 & 3.7 \\
0.10 & 0.460 & 2.17 & $<10^{-6}$ & 128.0 & 5.2 \\
0.10 & 0.440 & 3.28 & $<10^{-6}$ & 105.4 & 7.6 \\
\hline
\end{tabular}

TABLE 5-4

MODELS FOR $<-0.20$

\begin{tabular}{cccccc}
\hline$c$ & $\eta$ & $x_{c}$ & $V_{0}(\mathrm{~km} / \mathrm{s})$ & $V_{100}(\mathrm{~km} / \mathrm{s})$ & $R_{\mathrm{b}}(\mathrm{R})$ \\
\hline 0.20 & 0.495 & 1.04 & 10.1 & 261.0 & 2.8 \\
0.20 & 0.490 & 1.09 & 5.5 & 254.5 & 2.9 \\
0.20 & 0.480 & 1.21 & 0.8 & 241.5 & 3.3 \\
0.20 & 0.460 & 1.49 & 0.0004 & 217.1 & 4.0 \\
0.20 & 0.440 & 1.85 & $<10^{-6}$ & 194.2 & 4.8 \\
0.20 & 0.400 & 2.93 & $<10^{-6}$ & 153.1 & 7.3 \\
\hline
\end{tabular}


TABLE 5-5

MODELS FOR $=0.30$

\begin{tabular}{cccccc}
\hline$c$ & $\eta$ & $x_{c}$ & $V_{0}(\mathrm{~km} / \mathrm{s})$ & $V_{100}(\mathrm{~km} / \mathrm{s})$ & $R_{\mathrm{t}}(R)$ \\
\hline 0.30 & 0.495 & 1.02 & 10.8 & 335.2 & 2.9 \\
0.30 & 0.490 & 1.06 & 6.7 & 329.4 & 3.0 \\
0.30 & 0.480 & 1.13 & 1.7 & 317.9 & 3.2 \\
0.30 & 0.460 & 1.30 & 0.008 & 295.1 & 3.7 \\
0.30 & 0.440 & 1.51 & $1.2 \times 10^{-6}$ & 273.1 & 4.2 \\
0.30 & 0.400 & 2.06 & $<10^{-6}$ & 231.0 & 5.5 \\
\hline
\end{tabular}


It is a good Indication of $v_{0}$. The Increase of $V_{8}$ beyond 100R is small. The velocity at 200R. $v_{200}$, Is about $18-5$ higher than $v_{100}$. Columa 6 glves $R_{b}$. the radius at which $v_{100} / 2$ is reached. It is an Indication of how fast the radial velocity increases. If I approximate the velocity distribution by $\beta$ law veloc1ty, $V-V_{0}+\left(V_{0}-V_{0}\right)(1-l / x)^{\circ}$, there is a relationship betieen $R_{t}$ and $\beta$. A larger $R_{t}$ corresponds to a larger $\beta$ value and therefore a more slowly increasing velocity distribution.

It can be seer from the cables that in general for a fixed $e$, the sonic point Increases with decreasing $\eta$. The smaller the value of $c$, the faster the sonic point increases with the decrease in $\eta$. This can also be seen in Figure 5-2. The intelal velocity depends on both $c$ and $\eta$. For a fixed $C$. $V_{0}$ decreases with decreasing $\eta$; and for a fixed $\eta$. $V_{0}$ increases with increasing $\eta$. But $V_{0}$ is more sensitive to the variations of $t$ than to varlations of $\eta$. When changes from 0.01 to 0.03 for fixed $\eta-0.495$, $V_{0}$ changes from 2.6 to $10.8 \mathrm{~km} / \mathrm{s}$. In comparison, when $\eta$ changes from 0.495 to 0.480 for fixed $-0.01, V_{0}$ decreases sharply from 2.6 to below $10^{-}$ ${ }^{6}\left(3.9 \times 10^{-7}\right) \mathrm{km} / \mathrm{s}$. The velocity at $100 \mathrm{R}, V_{100}$. depends also on both 6 and $\eta$. As $\eta$ decreases while $C$ remains constant, $v_{100}$ decreases; and as $C$ increases while $\eta$ remalns the same, $V_{100}$ increases. In the ranges of $c$ and $\eta I$ considered, $v_{100}$ is more sensitive to the change of $\eta$. It can be seen that for a given $\epsilon, R_{b}$ decreases with the increase in $\eta$; and for a given $\eta$. $R_{b}$ increases with the decrease in $C$. For the range of 6 and $\eta$ considered, $R_{b}$ varies in the range of $2.9 R$ to $15 R$. For comparison, the typical value of $R_{\text {f }}$ fon the radiation driven wind model by optically thick lines is $2 R$.

The radial velocity distributions for $-0.01,0.05,0.10,0.20$ and 0.30 and different $\eta$ values are given in Figures $5-3,5-4,5-5,5-6$ and $5-$ 7 respectively. I can see fron these figures that some velocity curves 


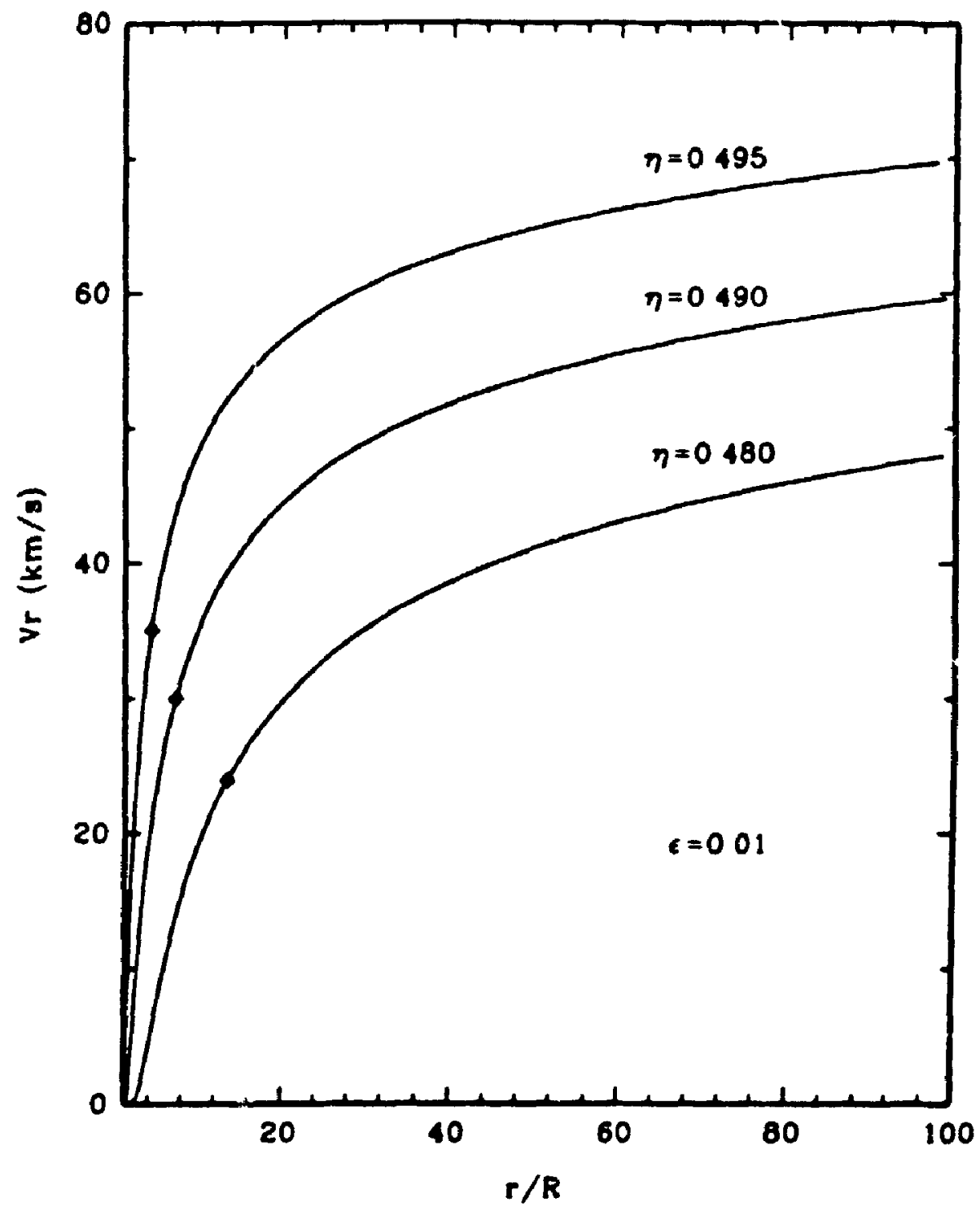

Figure 5-3. The radial veloctey distribution for $c-0.01$ and $n-0.495$, 0.490 and 0.480 . The diasonds Indicate the location of $R_{4}$, at which the velocity reaches half of $v_{100}$. 


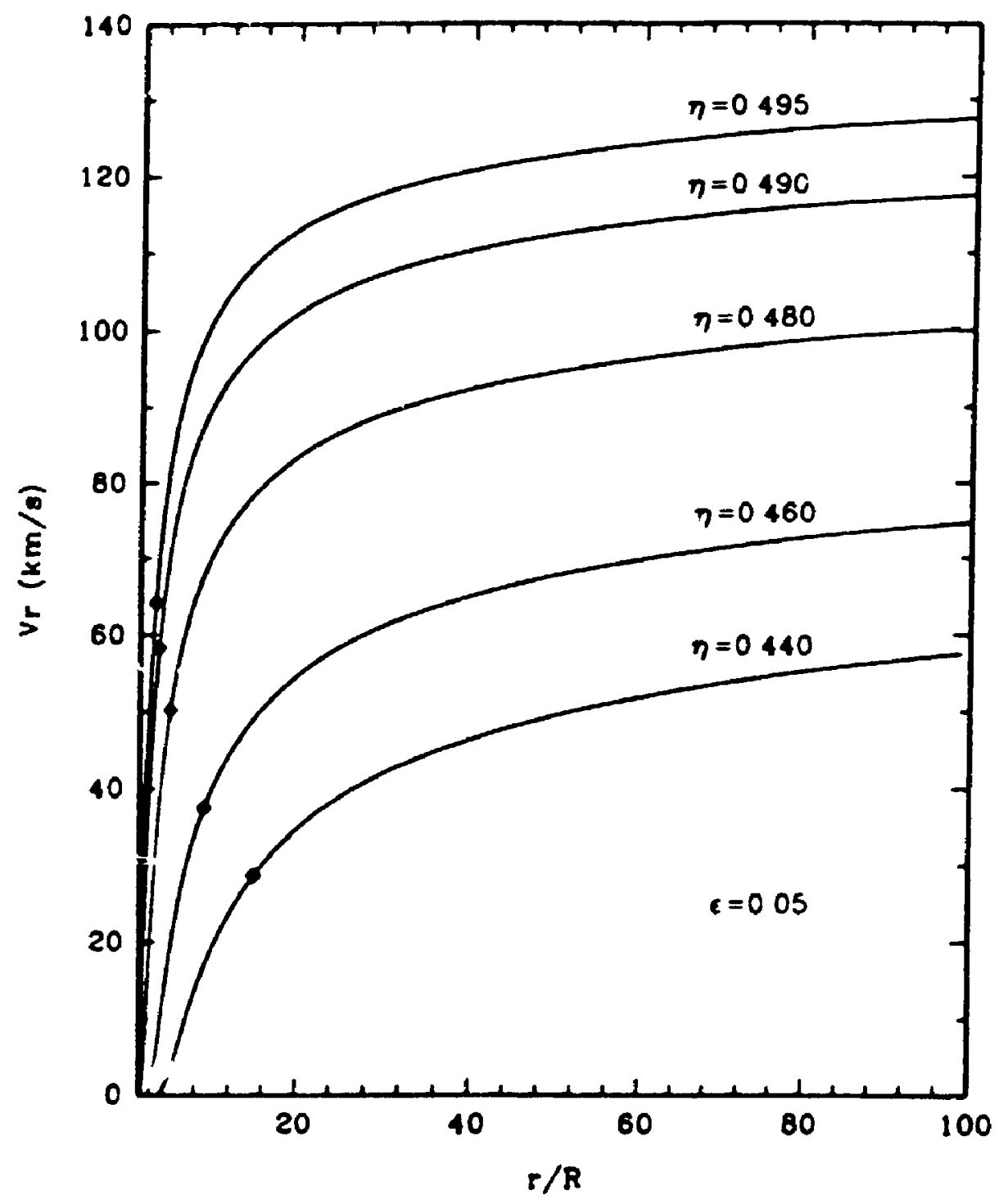

Figure $5-4$. The radial velocity distribution for $c-0.05$ and $\eta-$ $0.495,0.490,0.480,0.460$ and 0.440 . The diamonds indicate the location of $\mathrm{Rh}$, at which the velocity reaches half of $\mathrm{V}_{100}$. 


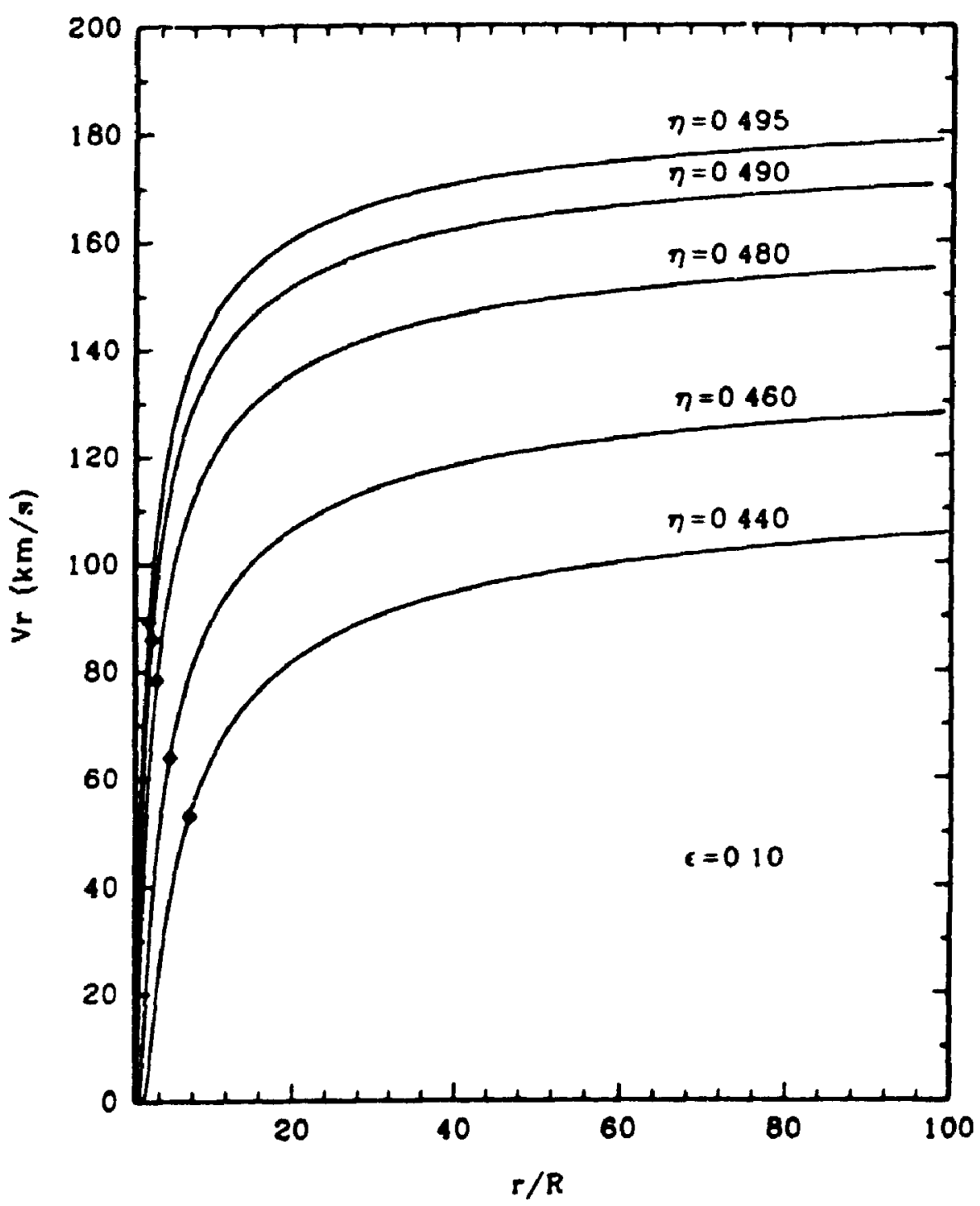

Figure 5-5. The radial velocity distribution for $c-0.10$ and $\eta-0.495$, $0.490,0.480,0.460$ and 0.440 . The diamonds Indicate the location of $R_{b}$, at whlch che velocity reaches half of $V_{100}$. 


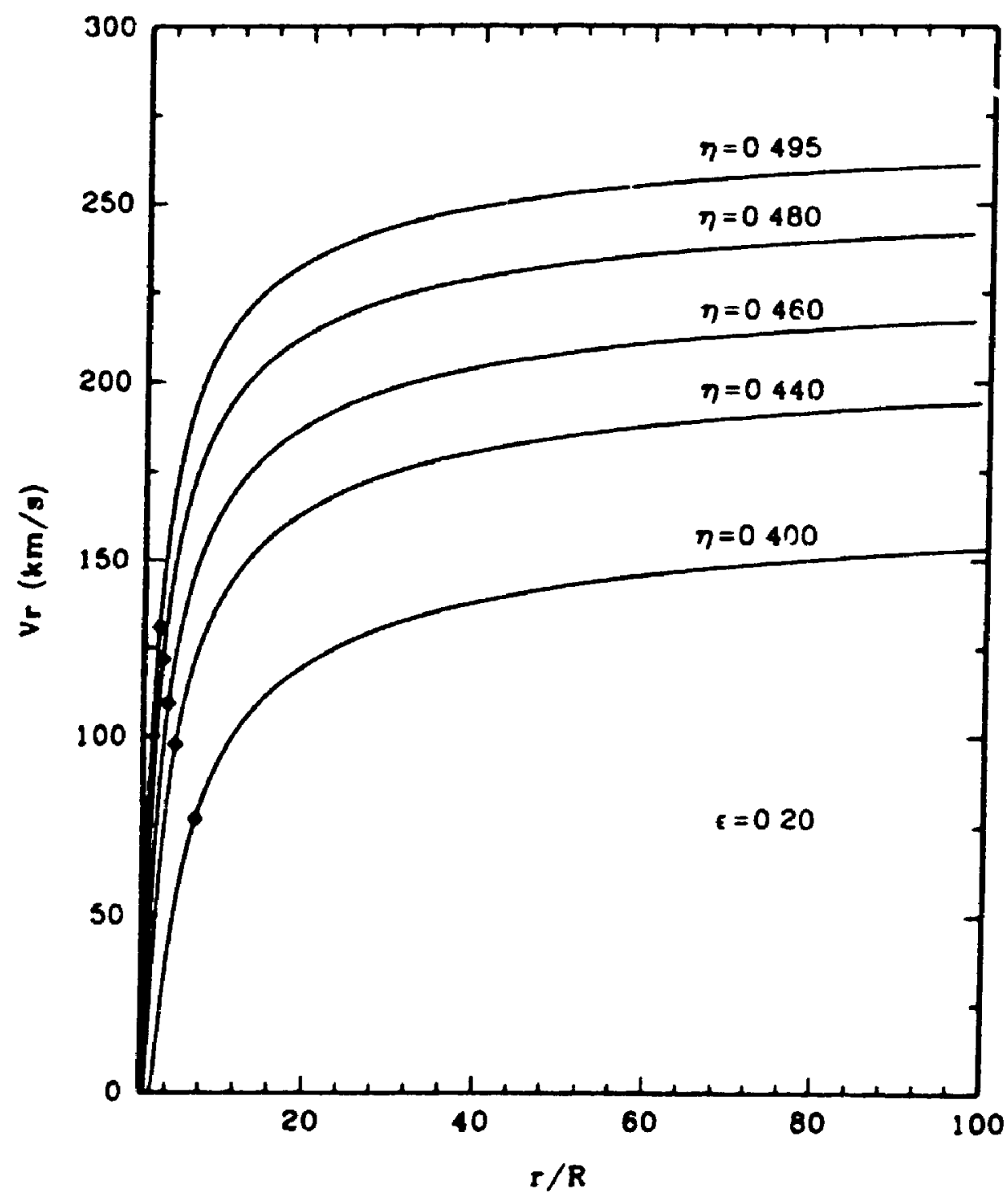

Figure 5-6. The radial velocley distribution for $c-0.20$ and $\eta-0.495$, $0.480,0.460,0.440$ and 0.400 . The dlamonds indicate the location

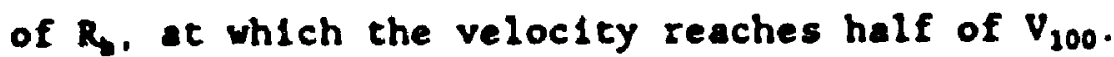




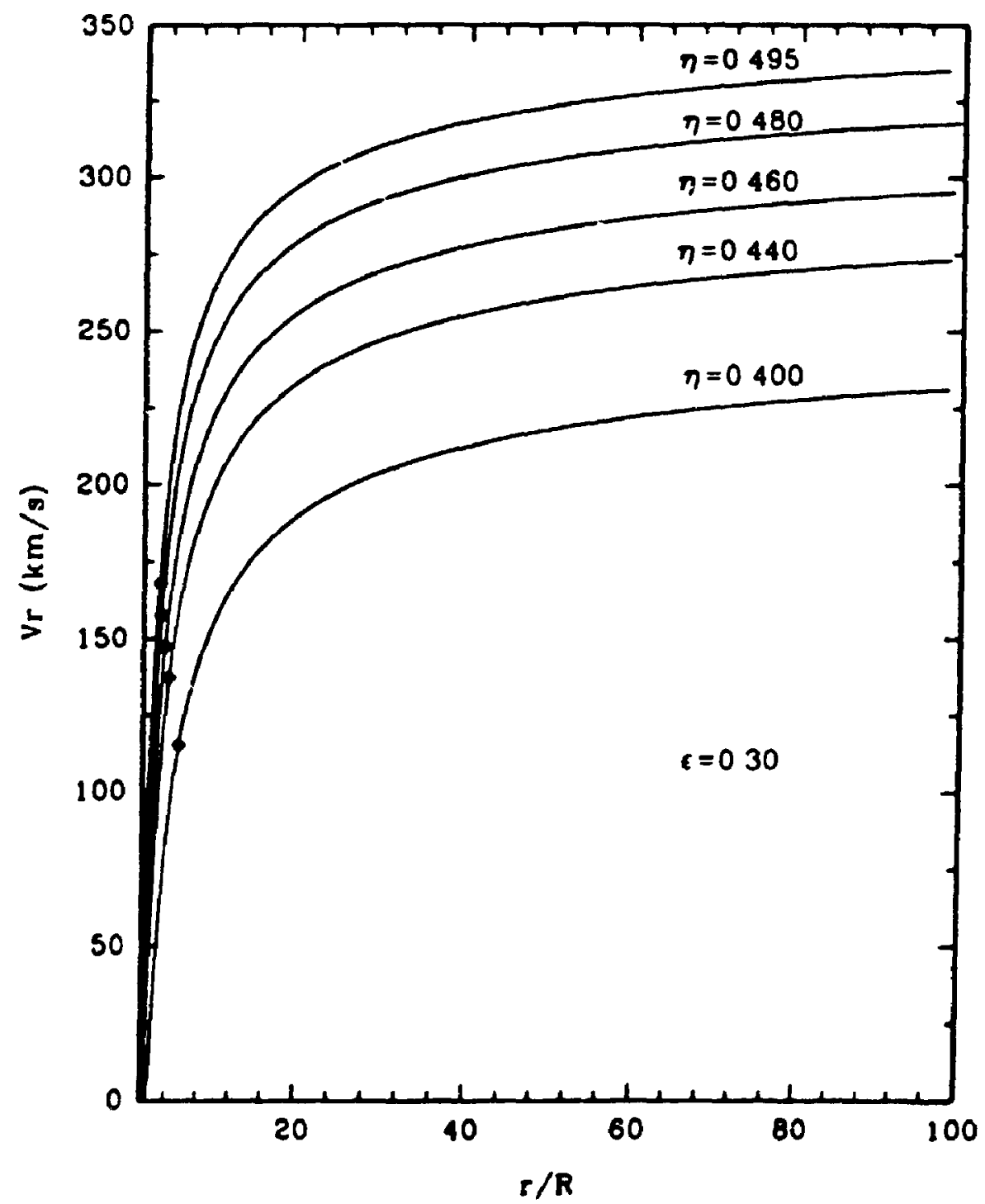

Figure 5-7. The radial velocity distribution for,-0.30 and $\eta-0.495$, $0.480,0.460,0.440$ and 0.400 . The diamonds Indlcate the location of $R_{0}$. at which the velocity reaches half of $v_{100}$. 
rises more rapidly than others. I use diamonds for the location of $R_{b}$.

\subsection{APPLICATION TO Be STARS}

As I mentioned before, one of the major difficulties in applying the strong line radiation driven model to Be stars is that the radial velocity from this wodel increases too rapidly. The emission lines produced in this wind will have $P$ Cyg type profiles. In contrast, the weak line driven model produces a wind with a much smaller velocity increase and terminal velocity if the values of $\epsilon$ and $\eta$ are chosen carefully.

In Figure 5-8, I give the velocity distribution produced by a strong line radiation driven model for $\gamma$ Cas with rotational velocity of $0.7 V_{b x a k-}$ up (indicated by "strong line" above the curve). In comparison, I also give the velocity distribution produced by a weak line model with $\epsilon=0.01$ and $\eta-0.495$. Since the region within the first 15 stellar radii contributes the most of emission (see PM), I only give the velocity distribution in the first 25 stellar radii. In fact, the radial velocity from a strong line radiation driven model has reached its terminal velocity at 25 stellar radii; and that from a weak line model is also close to its $V_{\bullet}$. The diamonds indicate the location of $R_{b}$ for both distributions. The velocity distribution for a strong line radiation driven model reaches half of its cerminal velocity at only 1.5 stellar radii compared with 4.4 for the weak line model.

Although the velocity distribution from a weak 1 ine model has a very low terminal velority, it still increases faster than that used in the PM model. The velocity distribution they used can be represented by $\beta$ law, described by equation (2-9), with $v_{0}-7.5 \mathrm{~km} / \mathrm{s}, V_{0}-253 \mathrm{~km} / \mathrm{s}$ and $\beta=$ 14.5. If I represent the velocity distribution from a weak line model 


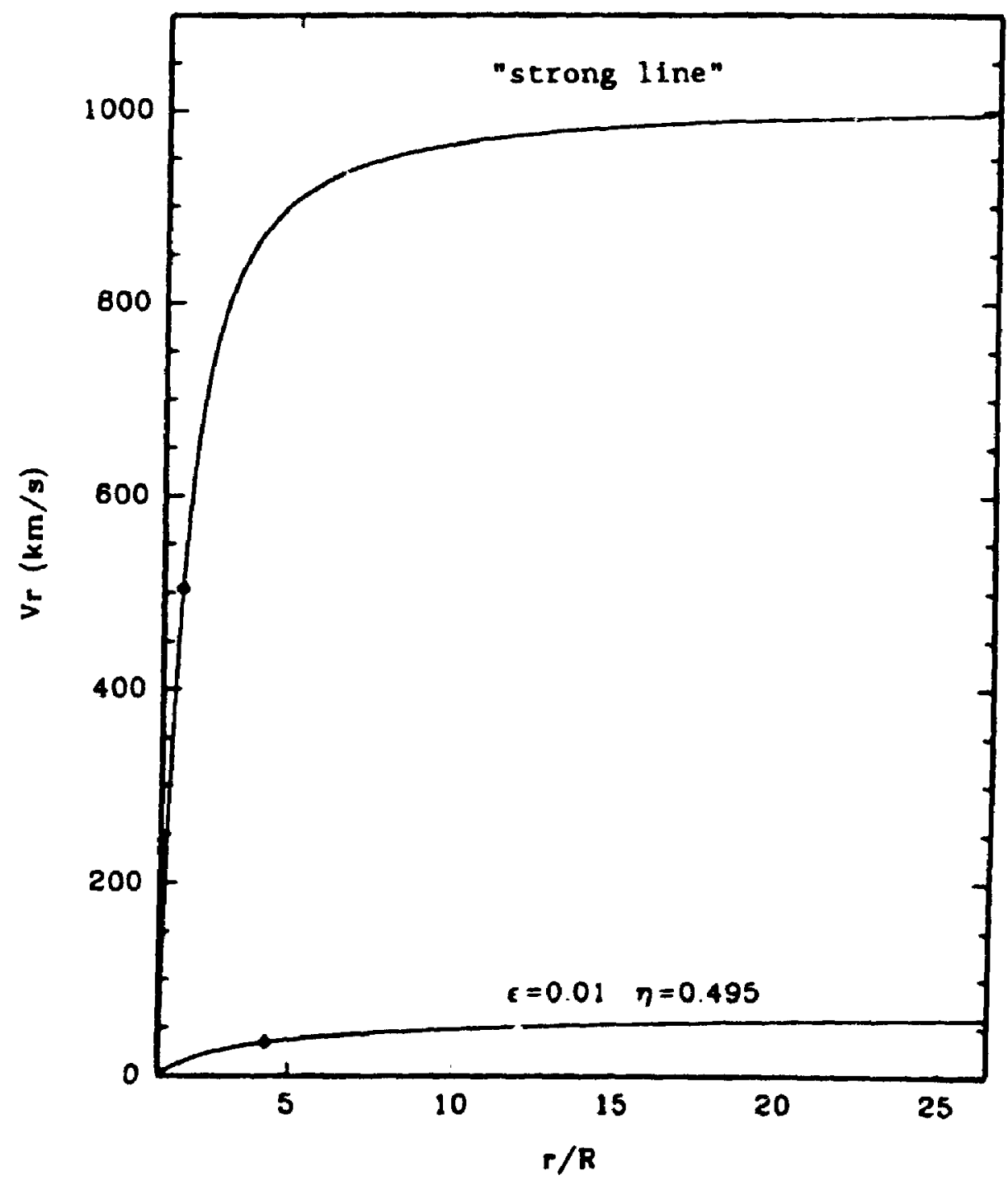

Figure 5-8. The comparison of radial velocity distribution from stong line driven wind model and that from our model with $c-0.01$ and $\eta=0.495$. The diamonds indicate the location of $R_{b}$, at whlch the velocity reaches $v_{100}$. 
with $C-0.01$ and $\eta-0.495$ by $\beta$ law, the value of $\beta$ is only 2.5 . I do not know whether a velocity distribution with a blgger $\beta$ but smaller terminal velocity is capable of reproducing the Ha emission profile as does the PM nodel.

To test if my model has the potential to account for Ha profiles, I calculate Ho profiles using the computer code developed by PM but replacing the velocity distribution of the PM model by that from my model with $\epsilon-0.01$ and $\eta-0.495$. The Ha profile is given in Figure 5-9 for the surface density $n_{0}-1.8 \times 10^{14}$. The equivalent width is $6.0 \mathrm{~A}$. The profile is a little narrower than that of the PM model. This is very likely due to the difference in rotational velocities adopted by the two models, 1.e., $569 \mathrm{~km} / \mathrm{s}$ in $\mathrm{PM}$ model and $392 \mathrm{~km} / \mathrm{s}$ in my model.

The observation of linear polarization of Be stars suggest that the envelopes of Be stars are of a disk-like shape, i.e., the material is concentrated toward the equatorial plane. Remembering that a strong line model produces a much higher velocity distribution, therefore, a lower density distribution compared with a weak IIne model, I assume that the weak line driving mechanism is the dominant one in the equatorial plane while a strong line driving mechanism is the dominant one at the polar regions. An overall density distribution is drawn in Figure 5-10 under this assumption. In this figure, the density on the equatorial plane is from a weak line model with $c-0.01$ and $\eta-0.495$; the density at the poles is frow a strong line driven model with zero rotation. The density at other values is derlved using the relation

$$
\rho(r, \theta)-\rho_{D}(r)-\left[\rho_{0}(r)-\rho_{p}(r)\right] \sin n^{0} \theta
$$




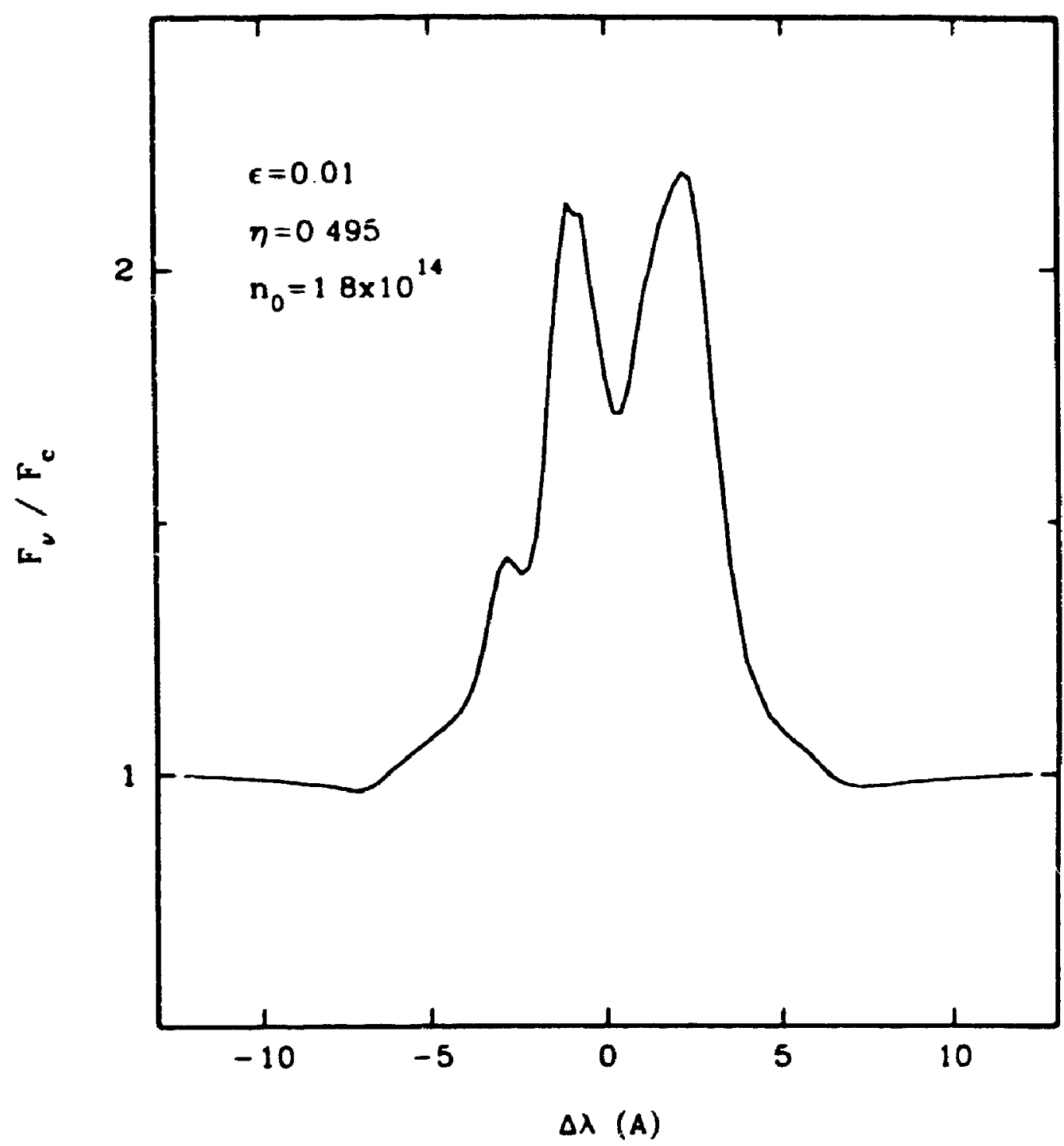

Figure 5-9. Ha profiles from PM model but the velocity distribution from my model with $c-0.01$ and $\eta-0.495$. The equivalent width is $6.0 \mathrm{~A}$ corresponding to the surface number density $1.8 \times 10^{14}$. 


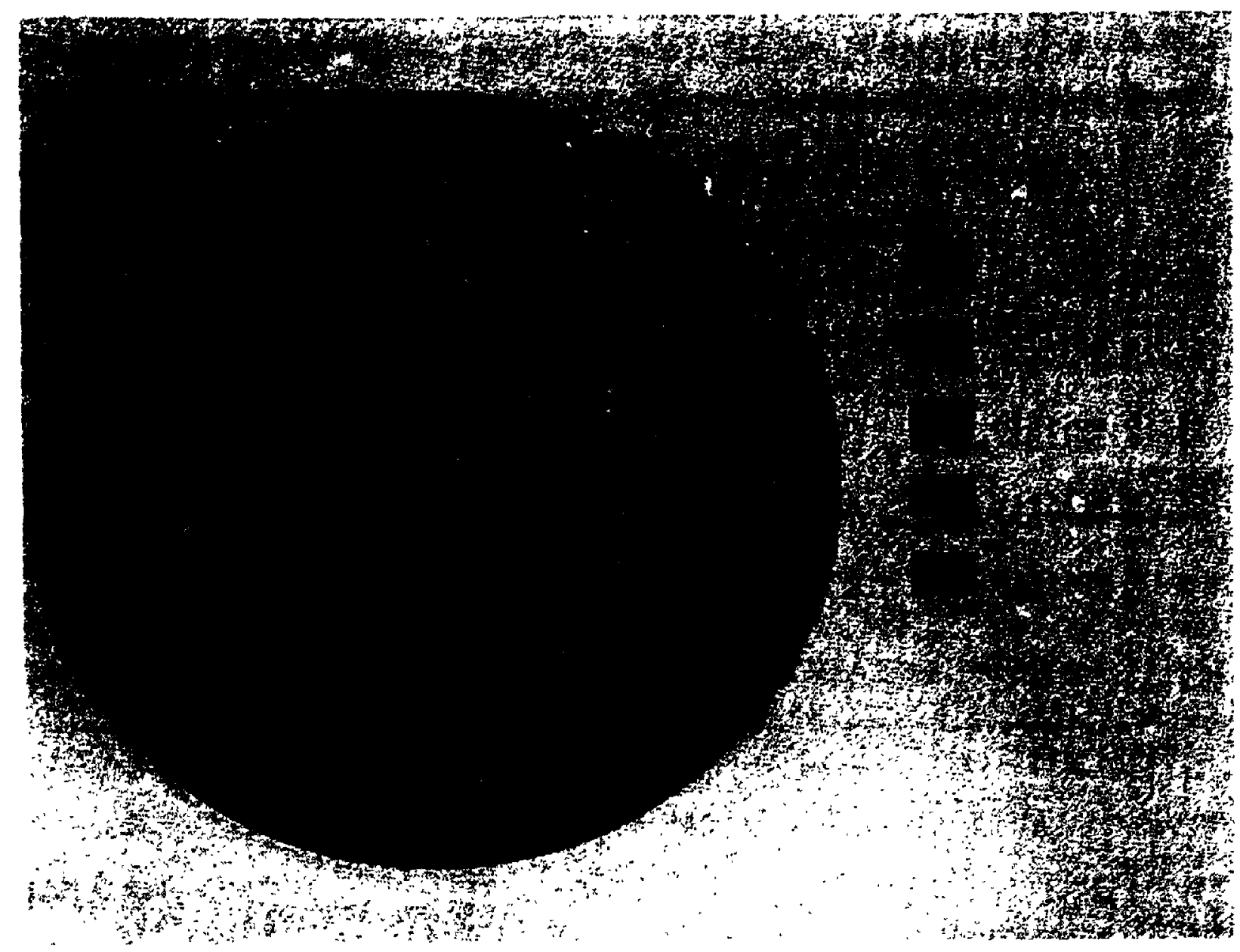

Figure 5-10. The cross section of a Be star envelope. The density distribution at equatorial plane, $\rho_{a}(r)$, is from weak line mode1 with $\epsilon=0.01$ and $\eta=0.495$ and the surface density is $1.8 \times 10^{14}$. The density at the pole, $p_{p}(r)$, is from strong line radiation driven mode1. The density at other places, $\rho(x, \theta)$, is determined by relation $\rho(r, \theta)-\rho_{p}(r)+\left[\rho_{b}(r)-\rho_{p}(r)\right] \sin ^{n} \theta$ (Boyd \& Marlborough 1991). In this picture, $n-6$. The letter $d$ in the figure has the same meaning as $f$ does. 
which was first used by Boyd \& Marlborough (1991). A disk-11ke envelope can be seen clearly from Figure 5-10. Although equation (5-46) might be too simple, the real situation will not differ too much as long as one believes the density changes continuously from the equatorial plane to the poles. 


\section{CHAPTER 6 CONCLUSIONS AND FUTURE WORK}

\subsection{CONCLUSIONS}

In this thesis, I have Investigated the dynamics of the envelopes of Be stars in the equatorial plane. Currently there is no general agreement as to what are the most important forces controlling the dynamics in and near the equatorial plane. In my approach, I Inverted the equation of motion and solved for the unknown force or forces, $F_{x}(r)$, in addition to gravity, rotation, and gas pressure gradient, required to produce a radial component of velocity having a functional form consistent with the observations.

All my investigations have indicated that $F_{x}(r)$ has a similar characteristic shape. Beginning at the surface of the star, $F_{x}(r)$ inf $=$ lally decreases with increasing $r$, but less rapidly than does gravity, reaches a minimum at 10-100 stellar radil, and increases again. It may continue to increase or reach a maximum and then decrease again, depending upon the detailed form of the velocity distribution used. For a $\beta$ law velocity distribution it increases again; and for a power law velocity distribution it continues to increase.

I then apply this scheme to six well studied Be stars using the results of Waters et al. (1991), among which three are Be stars and three are shell stars. The unknown force $F_{x}(r)$ for these six stars, normalized In terms of gravity, has two characteristics: a) between the surface of the star and the turning point, the normalized $F_{x}(r)$ is identical for all six stars; and b) the turning point occurs at smaller $r$ for Be stars than for shell stars. The first aspect suggests that the same driving 
mechanism dominates the dynamics interlor to the turning point. The second aspect implies that second driving wechanism takes over from the first one at smaller $r$ for Be stars than for shell stars. This difference In location of the onset of the second driving mechanism might be an explanation for why shell stars have more extensive envelopes than do Be stars.

I am hesitant to express this in a stronger way, not only because of the small sample of stars, but alse because of the well known variablifty of Be stars and of potential difficulty with transitions between Be and shell phases over the period in which the continum data were obtained.

I have discussed two possibilities for $F_{x}(r)$. One is the existence of a magnetic field. In this case, the angular momentum is transferred from the magnetic field to the material to support the envelope. And the other is the radiation force arising from a large number of optically thin lines.

In Chapter 4, I investigated the influence of a weak magnetic field on the Be envelopes, a possible driving mechanism proposed in Chapter 2. My investigations have shown that a weak magnetic field can influence the rotational velocity distribution quite effectively in a slowly expanding envelope by transferring angular momentum from the magnetic field to the material. For a $\beta-1$ aw velocity distribution with a large $\beta(-15)$, this transfer is so substantial that the wind is rotating at a speed faster than that of solid body rotation in the first few stellar radil.

For a power law velocity distribution, the azimuthal component of velocity is much higher than that for angular momentum conservation but lower than that for solid body rotation for all cases considered. When 
the velocity index and the mass loss rates are fixed, the azimuthal velocity Increases with increasing $B_{0}$; when the velocity index and $B_{0}$ are fixed, the azimuthal velocity increases with decreasing mass loss rate: and when $B_{0}$ and the wass loss rate are fixed the azimuthal velocity increases with decreasing velocity index.

In the radial direction, the radial component of the magnetic force is negligible for the strength of the magnetic field considered, But the centrifugal force is enhanced due to the transfer of the angular momentum to the material. For some cases, It is so large that the force itself can balance gravity.

In summary, a weak magnetic fleld can boost up dramatically the rotational velocity distribution of a slowly expanding wind; in some cases the rotational velocity becomes Keplerian so that the centrifugal force itself can support the envelope.

In chapter 5, I investigated the driving of the envelopes by the force arising from a large number of optically thin lines. I introduced two parameters $\epsilon$ and $\eta$ to describe the force, the former is a measure of the strength of the force at the surface of the star and the latter describes the dependence of the force on the distance. The requirement for the location of the sonic point provided some restriction on the values of and $n$.

I calculated the velocity distribution for different values of $\epsilon$ and $\eta$. Values of 6 and $\eta$ can be found so that the velocity distribution increases much more slowly than does the velocity for the strong line driven wind model; and the terminal velocity 1 les between 60 to $300 \mathrm{~km} / \mathrm{s}$ compared with over $1000 \mathrm{~km} / \mathrm{s}$ fron the strong line driven wind model. The 
calculations for the model with $\epsilon-0.01$ and $\eta-0.495$ and surface number density $\mathrm{n}-1.8 \times 10^{14} / \mathrm{cm}^{-3}$ produce a grossly symmetric profile with an equivalent width $-6 A$. This demonstrates that the weak Iine driven wind model is capable of reproducing the Ha emission profile as does the PM model.

I then assumed that the weak line driving mechanism is the dominant one in the equatorial plane while a strong line driving mechanism is the dominant one at the polar region. Under this assumption I calculated the overall density distribution. And a disk-like envelope was produced.

\subsection{FUTURE WORK}

My investigation concerning the unknown driving force of the envelopes of Be stars indicates that between the surface of the star and a turning point of 10-100 stellar radil, this force decreases with increasing $r$ but less rapidly than does gravity. Beyond the turning point this force increases with increasing $r$. The change in the force suggests that different forces dominate inside and outside the turning point. The investigation of the observed continuous energy distribution of Be stars by Taylor et al. (1987) shows that a turn-over in the continuum energy distribution occurs between the IR and radjo region. Is there any connection between the turning point in the unknown force and the change of slope of continum energy distribution? I Intend to calculate the continuous energy distribution of Be stars in order to see how the change in slope of energy distribution is related to the turning point of the unknown force. Understanding the connection between the turning point of the unknown force and the change in the continuum slope should lead to a 
better understanding of the circumstellar envelopes of Be stars.

The radiation force due to the optically thin lines is a very promising candidate for the unknown force responsible for the driving of Be star envelopes. In chapter 5, I constructed a model in which the radiation pressure due the optically thin lines is the maia driving force. My model can produce winds with terminal velocities around $100 \mathrm{~km} / \mathrm{s}$. The Ha profile from this wind for a star like $\gamma$ Cas agrees well with observations obtalned in 1973-1977. However the parameter, $W(r)$, which measures the strength of the optically thin line force is not obtained from first principles; instead it is empirical. I intend to consider the radiation processes in the envelope in more detail so that I will be able to derive a better expression for this parameter.

The CAK, strong-line driven wind model, encounters difficulties when applied to iapidly rotating stars. When the rotational velocity is higher than 708 of the break-up velocity, the numerical solution cannot be extended beyond a certain distance from the star. For a $\gamma$ Cas like star with $V_{\text {rat }}=0.75 V_{\text {breakup }}$, this point is located at about 10R. The higher the rotational velocity is, the closer is this point to the stellar surface. Current observations suggests that Be stars don't rotate faster than about 708 of the break-up speed. However gravity darkening complicates the picture. Becalt.e of gravity darkening, the observed $v$ sin $i$ are lower Iimits to the actual values of $v$ sin $i$; hence some Be stars may actually be rotating closer the break-up speed. My investigation about the locus of the singular points reveals that an additional branch of this locus appears when high rotation occurs. It is this new locus which stops the numerical solution. Also I find that there is one point on the new branch 
of the locus of singular points that could be chosen as the critical point on the second locus of singular points. It is likely that a wind with a low velocity distribution could be found, which could explain the dynanics Be star envelopes. I am going to carry out the detalled calculation to see if this approach does work. 
REFERENCES

Abbott, D. C. 1979, in IAU Symposium 83. Mass Loss and Evolution of o.Type Stars, eds. P. Cont1 \& C. de Loore (Dordrecht:Reidel), p. 317 .

Abbott, D. C. 1985, in Relations between Chromospheric-coronal Heating and Mass Loss in Stars, eds. R. Stalio \& J. Zirker, Trieste: Observatorio Astroriomico di Trieste, p. 265.

Bahng, J. D. 1976, In IAU Symposium No. 70, Be and Shell stars, ed. A. Slettebak, p. 41 .

Barker, P. K. 1987, In IAU Colloquium 92, Physics of Be Stars, eds. A. Slettebak \& T. P. Snow (Cambridge: Cambridge University Press), p. 38 .

Barker, P. K., \& Marlborough, J. M. 1982, Astrophy. J., 254, 297.

Beals, C. S. 1951, Pub. Dom. Ast. Obs., IX, 1.

Beeckmans, Fr. 1976, Astron. Astrophys., 52, 465.

Boyd, C. J. Marlborough, J. M. 1991, Astrophys. J., 369, 191.

Cassinel1i, J. P. 1985, in The Origin of Non-radiative Heating/Momentum in the Hot Stars, NASA CP 2358, ed, A. B. Underhill \& A. G. Michalitsianos, p. 2 .

Castor, J. I., Abbott, D. C., \& Klein, R. I. 1975, Astrophys. J., 19y 157.

Chen, H. \&uang, L. 1986, Acta Astrophysica Sinica, Vol, 6, No. 4, 271. Chen, H., Marlborough, J. M., \& Waters L. B. F. M. 1992, Astrophys. J., 384,605 .

Chen, H., \& Marlborough, J. M. 1992a, Astrophys. J., September 20.

Chen, H., \&arlborough, J.M. 1992b, In preparation. 
Corbet, R.H.D., Smale, A.P., Menzles, J.W., Branduard1-Raymont, G., Charles, P.A., Mason, K.O., Booth, L. 1986, Mon. Not. R. Astr. Soc., $221,961$.

Delplace, A. M., \& Hubert, H. 1975, Astron. Astrophys., 38, 75.

Doazan, v. 1976, in IAU Symposium No. 70, Be and Shell Stars, ed. A. slettebak, p. 37 .

Doazan, v. 1987, in IAU Colloquium 92, Physics of Be stars, eds. A. Slettebak, T. P. Snow, p. 364 .

Doazan, V. Thomas, R. N. 1982, in The Be Stars with and whout Emission Lines, eds. A. B. Underhill \& V. Doazen, NASA SP-456, P. 409 .

Friend, D. B., \& Abbott, D. C. 1986, Astrophys. J., 311, 701.

Gehrz, R. D., Hackwe11, J. A., Jones, T. W. 1974, Astrophys. J., $191,675$.

Gulliver, A. F. 1981, Astrophys. J., 24E, 222.

Hartmann, L., \& MacGregor, K. B. 1980, Astrophys. J., 242, 260.

Harmanec, P., 1982, in The Be Stars with and without Emission Lines, eds. A. B. Underhill \& V. Doazen, NASA SP-456, p. 279.

Hutchings, J. B. 1970, Mon. Not. R. Astr. Soc., 150, 55.

Hutchings, J. B. 1976, in IAUl Symposium No. 70, Be and Shell Stars, ed.

A. Slettebak (Dordrecht: ReIdel), p. 13.

Jaschek, M. 1982, IAU Symposium No 98, Be Stars, ed. M. Jaschek.

Johnson, H. L., Mitche11, R. I., Iriarte, B., Wisniewsk1, W. 2. 1966 , Comm. Lunar Plane. Lab., 4, 99.

Johnson, H. L. 1967, Astrophys. J. Letters, 150, L39.

Kudritzki, R. P. 1988, in Radiation in Moving Gaseous Media, Elghteenth Advanced Course, Swiss Society for Astrophysics and Astronomy, eds. 
Y. Chmlelewski \& I. Lanz (Geneva:Geneva Observatory), p. 3.

Lamers, H. J. G. L. M. 1986, Astron. Astrophys., 159, 90.

Lamers, H. J. G. L. M., Rogerson, J. B. 1978, Astron. Astrophys., 66, 417.

Limber, D. N., Marlborough, J. M. 1968, Astrophys. J., 152, 181.

Lucy, L. B. Solomon, P. M. 1970, Astrophys. J., 159, 879.

MacFarlane, J.J., \& Cassinelli, J.P. 1989, Astrophys. J., 347, 1090.

McLaughlin, D. 1961, JRASC, 55, 13 and 73.

Marlborough, J. M. 1976, in IAU Symposium No. 70, Be and Shell stars, ed. A. Slettebak, Dordrecht: Reide1, P. 335.

Marlborough, J. M. 1987, in IAV Colloquium 92, Physics of Be stars, eds. A. Slettebak \& T. P. Snow (Cambridge:Cambridge University Press), p. 316.

Marlborough, J. M., \& Cowley, A. P. 1974, Astrohysp. J., 187, 99.

Marlborough, J. M., \& Zamir, M. 1975, Astrophys. J., 195, 145.

Marlioorough, J. M., \& Zamir, M. 1984, Astrophys. J., 276, 706.

Merril1, P. W., and Burwell, C. G. 1950, Astrophys. J., 112, 72.

Mestel, L. 1968, Mon. Not. R. Astr. Soc., 138, 359.

Morton, D. C. 1976, Astrophys. J., 203, 386.

Parker, E. 1958, Astrophys. J., 128, 664.

Pijpers, F. F., \& Hearn, A. G. 1989, Astron. Astrophys., 209, 198.

Poe, C. H., \& Friend, D. B. 1986, Astrophys. J., 311, 317.

Poeckert, R. 1982, in IAU Symposium No. 98, Be Stars, eds. M. Jaschek \& H. G. Groth (Dordrecht:Reide1), p. 453.

Poeckert, R., \&arlborough, J. M. 1978, Astrophys. J., 220, 940.

Secch1, P. 1866, Comptes Rendus Acad. Sciences Paris, 63, 621.

Slettebak, A. 1976, IAU Symposium No. 70, Be and Shell stars, ed. A. 
Slettebak.

Slettebak, A. 1982, Astrophys. J. Supp1., 50, 55.

Slettebak, A., and Carpenter, K. G. 1983, Astrophys. J. Suppl., 53, 869.

Slettebak, A., Snow, T. P. 1987, IAU Colloquium No. 92, Physics of Be Stars (Cambridge: Cambridge University Press).

Slettebak, A., Collins II, G. W., and Truax, R. 1992, Astrophys. J. Supp1., 81, 335.

Struve, 0. 1931, Astrophys. J., 73, 94 .

Swank, J.M. 1985, in The Origin of Nonthermal Heating/Momentum in Hot Stars, eds. A.B. Underhill \& A.G. Michalitsionos (NASA Pub 2358). p. 86 .

Taylor, A. R., Waters, L. B. F. M., Lamers, H. J. G. L. M., Persi, P.. \& Bjorkman, K. S. 1987, Mon. Not. R. Astr. Soc., $228,811$.

Taylor, A. R., Waters, L. B. F. M., Bjorkman, K. S., \& Dougherty, S. M. 1990, Astron. Astrophys., 231, 453.

Telting, J.H., Waters, L.B.F.M., Persi, P., and Dunlop, S. 1991, in Eso Workshop on Rapid Variability of OB Stars: Nature and Diagnostic Value, ed. D. Baade (Garching bel Munchen, ESO), p. 57.

Underhill, A., \& Doazan, V. 1982, B Stars With and Without Emission Lines (Washington: NASA. SP-465), P. 357.

Uesugi, A., \& Fukuda, I. 1982, Revised Cat. of Stellar Rotational Velocities, Kyoto University.

van den Heuve1, E. P. J., Rappaport, S. 1987, In IAU Colloquium No. 92, Physics of Be Stars, eds. A. Slettebak and T. P. Snow, p. 291. Wackerling, I. R. 1970, Mon. Not. R. Astr. Soc., 73, 153.

Waters, L. B. F. M. 1986, Astron. Astrophys, 162, 121.

Waters, L. B. F. M. 1991, private communication. 
Waters, L. B. F. M., Cote, J. Aumann, H. H. 1987, Astron. Astrophys., 172, 225 .

Waters, L. B. F. M., Cote, J., L Lamers, H. J. G. L. M. 1987, Astron. Astrophys., 185, 206.

Waters, L. B. F. M., Taylor, A. R., van den Heuvel, E. P. J., Habets, G. M. H. J..\& Persi, P. 1988, Astron. Astrophys., 198, 200.

Waters, L. B. F. M., van der Veen, W. E. C. J., Taylor, A. R., Marlborough, J. M., \& Dougherty, S. M. 1991, Astron. Astrophys., 244, 120 .

Weber E., \& Davis L. 1967, Astrophys. J., 148, 217. 